\title{
WAVEFRONT SHAPING MICROSCOPY
}

$$
\begin{aligned}
& \ldots \text { Controlling scattered light } \\
& \text { for deep tissue imaging }
\end{aligned}
$$

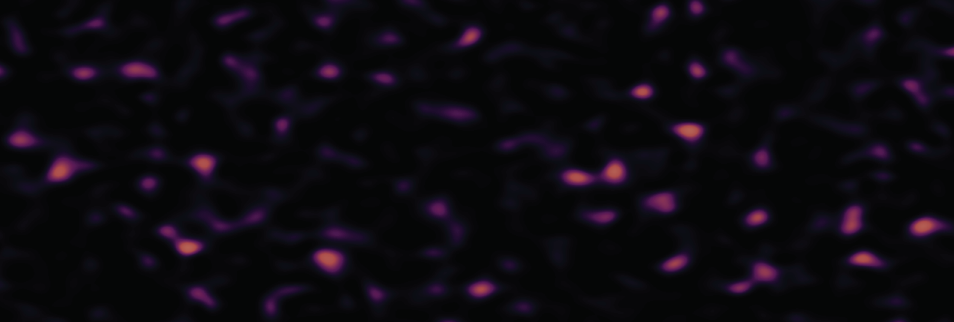

Gerwin Osnabrugge 


\section{WAVEFRONT SHAPING MICROSCOPY}

Controlling scattered light for deep tissue imaging

Gerwin Osnabrugge 


\section{Promotiecommissie}

Voorzitter: $\quad$ Prof. dr. J.L. Herek

Promotor: $\quad$ Prof. dr. I.M. Vellekoop

Co-promotor: Prof. dr. ir. W. Steenbergen

Overige leden: Prof. dr. S. Gigan

Prof. dr. P.W.H. Pinkse

Prof. dr. S. Stallinga

Dr. ir. H.L. Offerhaus

The work in this thesis was carried out at the Biomedical Photonic Imaging group of the Faculty of Science and Technology of the University of Twente.

The research leading to these results has received funding from the European Research Council (ERC-2016-StG-678919).

Cover design: Gerwin Osnabrugge

Printed by: Gildeprint

ISBN: $\quad$ 978-90-365-5017-8

DOI: $\quad$ https://doi.org/10.3990/1.9789036550178

(C2020 Gerwin Osnabrugge, The Netherlands. All rights reserved. No parts of this thesis may be reproduced, stored in a retrieval system or transmitted in any form or by any means without permission of the author. 


\section{WAVEFRONT SHAPING MICROSCOPY: CONTROLLING SCATTERED LIGHT FOR DEEP TISSUE IMAGING}

\section{PROEFSCHRIFT}

ter verkrijging van

de graad van doctor aan de Universiteit Twente, op gezag van de rector magnificus,

Prof. dr. T.T.M. Palstra,

volgens besluit van het College voor Promoties

in het openbaar te verdedigen op

vrijdag 10 juli 2020 om 14:45 uur

\section{door \\ Gerwin Osnabrugge}

geboren op 29 maart 1991

te Scherpenzeel, Nederland 
Dit proefschrift is goedgekeurd door:

Prof. dr. I.M. Vellekoop

en

Prof. dr. ir. W. Steenbergen 


\section{Contents}

1 Introduction 1

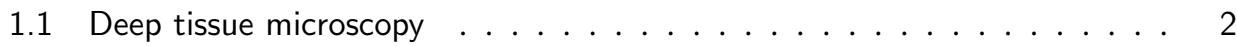

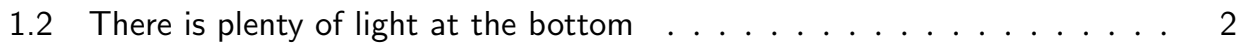

1.3 Reversal of light scattering . . . . . . . . . . . . . 3

1.4 A guide through this thesis $\ldots \ldots \ldots \ldots \ldots \ldots$

2 Blind focusing through strongly scattering media using wavefront shaping $\begin{array}{ll}\text { with nonlinear feedback } & 9\end{array}$

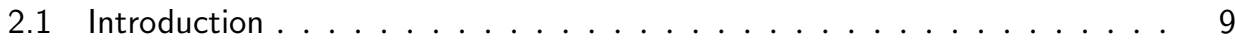

2.2 Theory of blind focusing . . . . . . . . . . . . . . . 11

2.3 Statistical model for blind focusing . . . . . . . . . . . . . . 12

2.4 Experimental validation . . . . . . . . . . . . . . . . . 14

2.4 .1 Experimental setup . . . . . . . . . . . . . . . . . . 14

2.4.2 Blind focusing experiment with two feedback targets . . . . . . . 14

2.5 Blind focusing requirements $\ldots \ldots \ldots \ldots \ldots \ldots \ldots$

2.5 .1 Minimal requirements . . . . . . . . . . . . . . . 15

2.5.2 Blind focusing experiment with multiple targets . . . . . . . . 17

2.5.3 Breakdown of blind focusing . . . . . . . . . . . . . 19

2.6 Discussion . . . . . . . . . . . . . . . . . . . . . . . 19

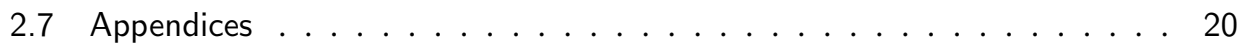

2.7.1 Convergence to local maximum . . . . . . . . . . . . . 20

2.7.2 Derivation of the blind focusing statistical model . . . . . . . 23

2.7.3 Derivation of the blind focusing requirements . . . . . . . 25

3 The generalized optical memory effect 29

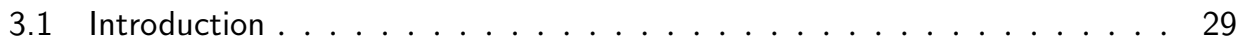

3.2 Model . . . . . . . . . . . . . . . . . . . . . . . . . . 30

3.3 Approximate solution for forward scattering . . . . . . . . . 33

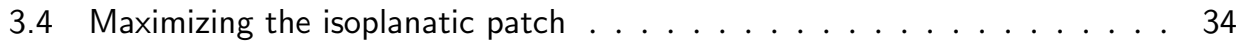

3.5 Experimental validation . . . . . . . . . . . . . . . 35

3.5.1 Measurements of the light field transmission function . . . . . . . 36

3.5.2 Measurements of the generalized correlation function . . . . . . . 38

3.6 Discussion . . . . . . . . . . . . . . . . . . . . . . 38

3.7 Appendices . . . . . . . . . . . . . . . . . 40

3.7.1 Wigner input-output derivation . . . . . . . . . . . 40 
3.7.2 Fokker-Planck model ................... 41

3.7.3 Correlation measurement .................. 45

4 Wavefront shaping two-photon microscopy 49

4.1 Two-photon excitation microscopy ................ . . 49

4.2 Focus optimization using fluorescence feedback . . . . . . . . . . . . 51

4.2.1 Localized source of feedback . . . . . . . . . . . . . . 51

4.2.2 Two-step optimization algorithm . . . . . . . . . . . . . . 51

4.2 .3 Focusing experiment ................................. 51

4.3 Enhanced imaging through a scattering layer . . . . . . . . . . . . . 52

4.3 .1 Conjugate adaptive optics . . . . . . . . . . . . . . 52

4.3.2 Digital conjugate adaptive optics . . . . . . . . . . . . 53

4.3.3 Proof-of-principle imaging experiment . . . . . . . . . . . . . 54

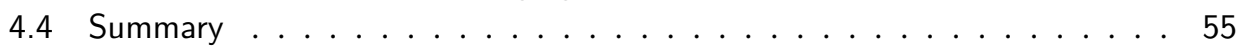

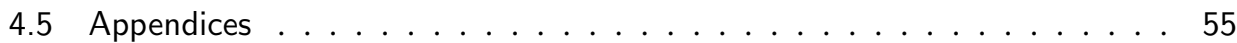

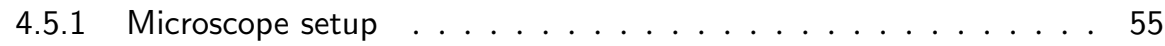

4.5 .2 Sample preparation ................... 57

5 Model-based wavefront shaping microscopy $\quad \mathbf{6 1}$

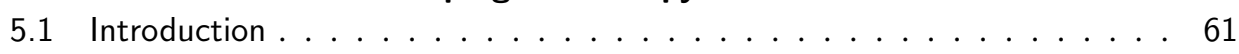

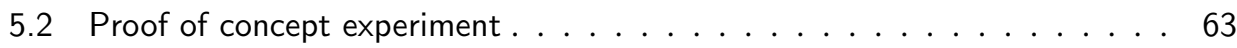

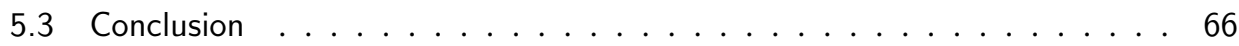

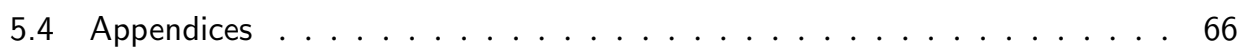

5.4 .1 3D Refractive index modeling . . . . . . . . . . . . 66

5.4 Light propagation simulations . . . . . . . . . . . . . 67

5.4.3 Calibration measurements for mapping SLM pixels to pupil plane . 69

6 A convergent Born series for solving the inhomogeneous Helmholtz equation in arbitrarily large media

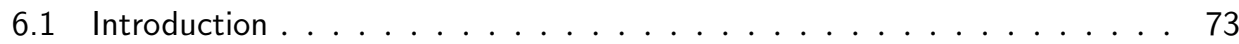

6.2 Traditional Born series . . . . . . . . . . . . . . . . . 74

6.3 Modified Born series . . . . . . . . . . . . . . . . . . . . . . . . . . . . . . . 75

6.3.1 Convergent Born series . . . . . . . . . . . . . 75

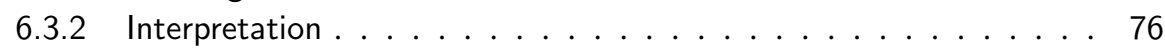

6.4 Implementation . . . . . . . . . . . . . . 78

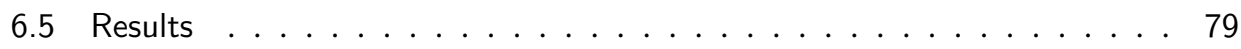

6.5 .1 Homogeneous medium . . . . . . . . . . . . . . . 79

6.5.2 Disordered inhomogeneous medium ............ . 81

6.5.3 Phase conjugation numerical experiments in adipose tissue. . . . . 82

6.5 .4 Three-dimensional media . . . . . . . . . . . . . . . 84

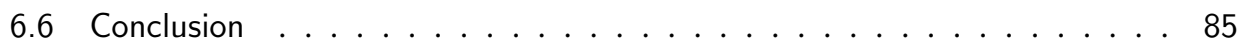

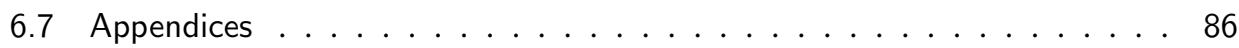

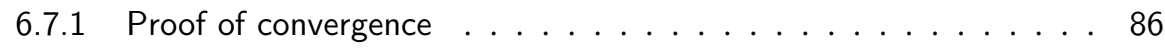

6.7 .2 Boundary conditions ................... 87 
7 Efficient boundary conditions for high-accuracy simulations of light propagation

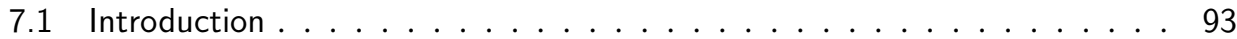

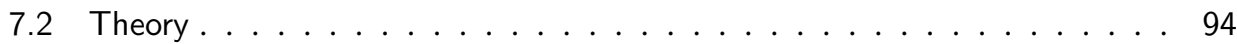

7.2.1 Modified Born series . . . . . . . . . . . . . . . . . . . 94

7.2.2 Anti-cyclic convolution ........................... 95

7.3 Implementation . . . . . . . . . . . . . . . . . 97

7.3.1 Pipelined MBS algorithm . . . . . . . . . . . . . . . . 97

7.3.2 Anti-reflection boundary layer . . . . . . . . . . . . . . . 98

7.4 Performance test . . . . . . . . . . . . . . . . . . 98

7.5 Conclusion . . . . . . . . . . . . . . . . . . 100

8 Summary and outlook 103

$\begin{array}{ll}\text { Nederlandse samenvatting } & 107\end{array}$

$\begin{array}{ll}\text { Acknowledgements } & 109\end{array}$ 


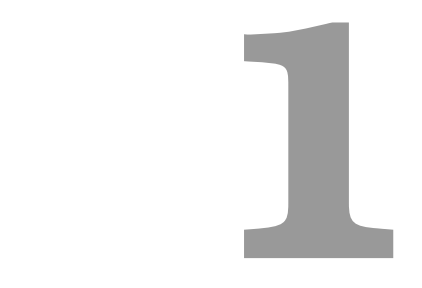

\section{Introduction}

"Seeing is believing". Sight is arguably the most important of all human senses. It allows us to recognize people and objects in the world around us based on their size, shape and color. With the introduction of powerful imaging instruments, our vision has been enhanced to the point that a black hole at millions of light years distance [1] and single atoms [2] can now be directly observed.

An open problem in imaging, however, is the inability to look inside or through opaque materials. A ground-based telescope cannot capture an image of the night sky on a cloudy night (see example in Fig. 1.1a). Similarly, as demonstrated in Fig. 1.1b, an object placed inside a glass of water becomes harder to see as more and more corn starch is added. Countless microscopic particles in these opaque materials reflect, refract and diffract the light in a disordered manner. These complex interactions of light and matter, known as light scattering, prevent us from imaging inside these materials.

The human body is such an opaque material. Imaging inside the body is vital to modern medicine, allowing for early diagnosis, tracking of disease progression and monitoring of brain activity. For medical imaging modalities such as X-ray computed tomography and magnetic resonance imaging the human body is mostly transparent. Therefore, these techniques can be used to obtain macroscopic images of organs located deep in the human body.

For observing individual cells and sub-cellular structures, optical microscopes have been the fundamental tool for centuries. In addition to their microscopic resolution, these tools provide a high sensitivity to low molecular concentrations from which detailed functional information can be extracted [3]. However, most microscopes require tissue to be extracted from the body and sliced up into thin samples. 

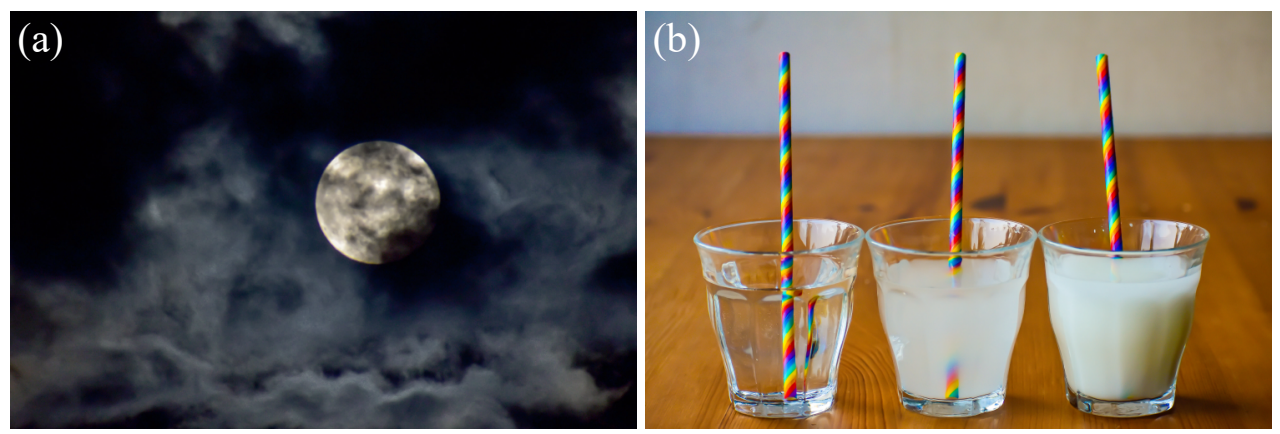

Figure 1.1: Examples of light scattering in daily life. (a) A full moon partially hidden behind passing clouds. (b) Paper straws placed in glasses of water with corn starch. From left to right, the concentration of corn starch is increased.

\subsection{Deep tissue microscopy}

Studying cells in their natural environment is of crucial importance to biological research [4]. For example, calcium imaging in a mouse brain could reveal how complex brain functions arise from the interactions between individual neurons [5]. For this reason, the last decades have seen the rise of many new imaging techniques pushing the depth limitation of optical microscopes.

To form a sharp image, microscopes rely on light to propagate in straight lines. However, the inhomogeneous structures inside the specimen itself will unavoidably scatter a part of the light. As a solution to this problem, deep tissue microscopy techniques have a variety of different mechanisms to isolate the non-scattered light. In confocal microscopy, for example, most of the scattered light is physically filtered out by placing a small pinhole in the detection pathway [6]. Other deep tissue microscopy techniques include optical coherence microscopy [7], photoacoustic microscopy [8] and multiphoton microscopy [9]. However, light scattering remains the major roadblock for deep tissue microscopy. Beyond a certain depth limit, the state-of-the-art microscopy techniques fail to capture high-resolution images of structures hidden inside scattering samples. In biological tissue this point is typically reached at a depth of approximately 1 millimeter [10].

\subsection{There is plenty of light at the bottom}

The imaging depth of deep tissue microscopes is limited by the amount of light that can be focused inside the sample. In Fig. 1.2, we simulated a light beam focusing through a set of scattering media with different thicknesses. As shown in Fig. 1.2b, when light propagates through a thin scattering medium, the focus becomes distorted. These distortions affect the resolution and contrast of the resulting microscope images. As the thickness of the scattering medium increases, the light is spread over a larger area and forms a complex interference pattern rather than a focus (see Fig. 1.2c).

The amount of non-scattered, or ballistic, light depends on the scattering length $\ell_{s}$. This property of the scattering medium represents the average propagation distance between two 

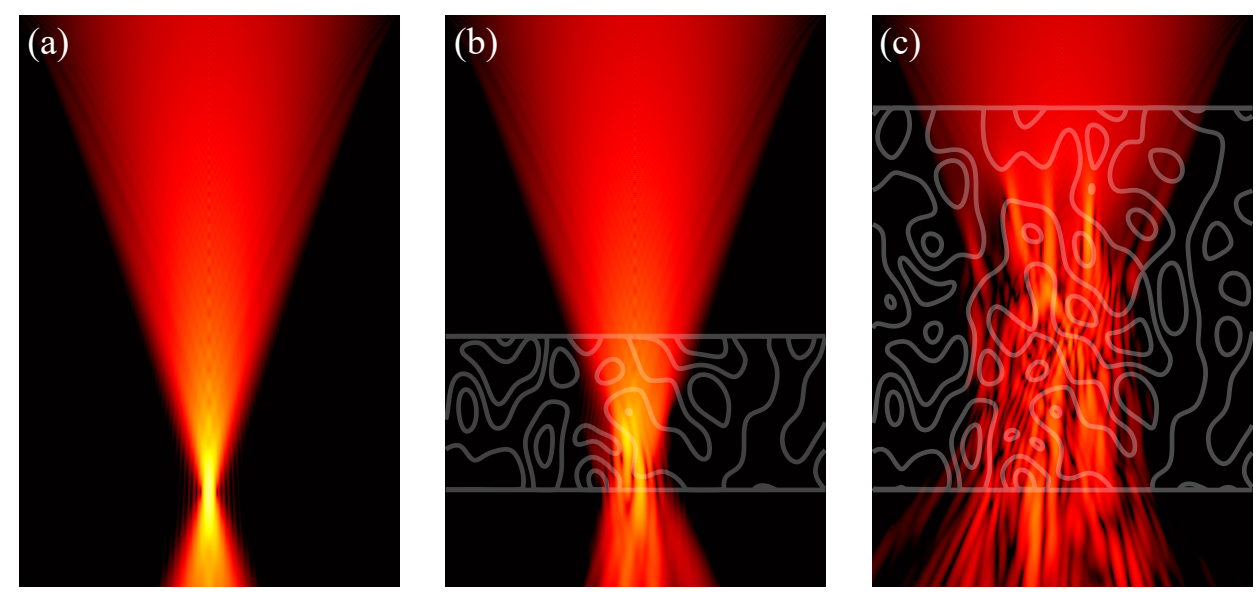

Figure 1.2: Simulation of light focusing light (a) a homogeneous medium, (b) a thin scattering layer and (c) a thick scattering layer. The gray lines represent the contour lines of the scattering structure. The inhomogeneities in the structure distort the light and prevent a focus from being formed. These simulations were performed using our new numerical method, which is discussed in Chapter 6.

consecutive scattering events. From Lambert-Beer's law [11], we know that the intensity of the ballistic light

$$
I_{b a l} \propto \exp \left(-\frac{z}{\ell_{S}}\right)
$$

where $z$ is the depth inside the scattering medium. For visible light, $\ell_{s}$ ranges from 50 to $100 \mu \mathrm{m}$ in typical biological tissue [12]. For example, when focusing light to a depth of 1 millimeter inside brain tissue, only ten parts per million will be ballistic.

In a thick scattering layer, the light does no longer form a focus, but a large portion of the light still propagates through the layer, as can be seen in Fig. 1.2c. Rather than the exponential decay of $I_{b a l}$, the total light intensity propagating to a depth of $z$ [13]

$$
I_{t o t} \propto \frac{\ell_{t r}}{z} .
$$

Here, $\ell_{t r}$ is known as the transport mean free path and describes the distance light can propagate through the medium before becoming diffusely scattered. In this multiple scattering regime, light is completely scrambled, yet still penetrates up to several centimeters deep into tissue [14]. Being able to control this diffuse light would open up revolutionary imaging possibilities inside the human body at microscopic resolution.

\subsection{Reversal of light scattering}

Light is an electromagnetic wave, and its propagation is described by the wave equation. A fundamental property of the wave equation is time reversal symmetry. A valid solution to the wave equation also yields a valid solution when played back in time. In other words, a wave 

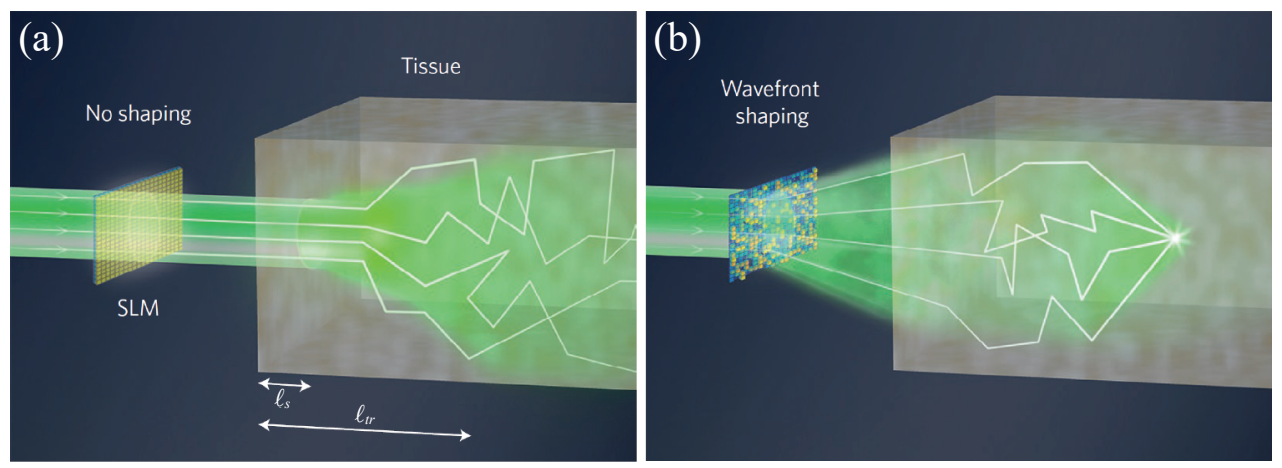

Figure 1.3: Basic principles of wavefront shaping. (a) A light beam is scattered as it propagates through biological tissue. (b) Light scattering can be compensated by controlling the wavefront of the incident light with a spatial light modulator (SLM). Once the correction wavefront is known, a focus can be formed at the desired depth inside the sample. Reproduced from Ref. [23] with permission.

propagating through a complex system could be sent back to retrace the same path through the medium and end up at the location it originated from $[15,16]$.

From time reversal symmetry, it becomes apparent that wave scattering is a deterministic process which could potentially be reverted. In optics, this was first realized by Vellekoop and Mosk [17] in a pivotal experiment, where light was shaped to focus through a strongly scattering layer of white paint. Step by step, the wavefront of the incident light was shaped to optimize the light intensity at a detector located behind the sample. Shortly after, this concept was extended to measure the transmission matrix of a disordered medium [18], allowing for an even higher degree of control over the scattered light. These pioneering experiments gave birth to a new field in optics known as complex wavefront shaping [19, 20].

With its potential to focus light deep inside tissue (see Fig. 1.3), wavefront shaping and the closely related field of adaptive optics [21] have been applied in a wide range of microscopy techniques [22-25]. These new techniques have shown promising results, yet their impact on in vivo applications remains limited to this date [26]. This can partly be attributed to the lack of a localized form of feedback inside the sample, which is required to form a focus. Also, scanning the optimized focus to acquire an image is not straight forward, as a different wavefront is needed to focus at a different location in the scattering sample. These challenges, together with several other technical and fundamental challenges, need to be overcome before wavefront shaping microscopy can be used for high-resolution imaging in the multiple scattering regime [27].

\subsection{A guide through this thesis}

In this thesis, we explore how the fundamental imaging depth limit of deep tissue microscopy can be tackled with wavefront shaping. We aim to achieve this by studying light scattering in tissue-mimicking samples and by developing new wavefront shaping microscopy techniques. Although most of the concepts studied in this thesis are relevant to deep tissue microscopy 
in general, we decided to focus our attention on two-photon excitation microscopy (TPM). TPM was chosen for its high contrast, limited photo-bleaching and the extensive variety in available fluorescent probes. The thesis is divided into three parts: (1) scattering theory, (2) wavefront shaping microscopy and (3) scattering simulations.

In the first part of the thesis, we will form new theoretical models for light scattering phenomena relevant to microscopy. In Chapter 2, we study how light can be focused through a scattering medium in the absence of a localized form of feedback. We will derive under which conditions the 'blind' optimization of a nonlinear feedback signal from multiple sources will lead to a single focus. In Chapter 3, we introduce the generalized optical memory effect, describing all first-order spatial correlations of scattered light. This correlation function describes how much an optimized focus can be shifted and tilted inside a scattering medium. From this unified correlation function, we derive the optimal scanning scheme for imaging inside forward scattering media.

In the second part of the thesis, we will discuss how wavefront shaping can be implemented to enhance the performance of a two-photon excitation microscope. Chapter 4 covers the basics of TPM imaging and how the embedded fluorescent structures can be used as feedback for the wavefront shaping algorithm. Furthermore, we demonstrate how the effective field of view through a scattering layer can be greatly enhanced using the generalized optical memory effect. In Chapter 5, we introduce a new class of wavefront shaping techniques, which we term model-based wavefront shaping. Instead of using the signal from a feedback source inside the scattering structure, this technique utilizes light propagation simulations to obtain the wavefront correction. This wavefront can then be constructed with the spatial light modulator in the TPM setup to form a sharp focus. In a proof-of-principle experiment, we demonstrate high-resolution imaging at depths where feedback can no longer be detected.

In the third part of the thesis, we will present a new numerical method for simulating light propagation in large scattering media. In Chapter 6 , we show that our method, based on a modified Born series, is orders of magnitude faster and more accurate than the current state-of-the-art methods. New boundary conditions for the numerical method are introduced in Chapter 7, further increasing the accuracy and the memory efficiency of the method.

\section{Bibliography}

[1] EHT collaboration et al., "First M87 Event Horizon Telescope results. I. The shadow of the supermassive black hole”, Astrophys. J. Lett. 875, 1-17 (2019).

[2] Y. Jiang, Z. Chen, Y. Han, P. Deb, H. Gao, S. Xie, P. Purohit, M. W. Tate, J. Park, S. M. Gruner, V. Elser, and D. A. Muller, "Electron ptychography of 2D materials to deep sub-ångström resolution”, Nature 559, 343-349 (2018).

[3] C. Boudoux, Fundamentals of biomedical optics (Blurb) (2017).

[4] V. Ntziachristos, "Going deeper than microscopy: the optical imaging frontier in biology”, Nat. Methods 7, 603-614 (2010).

[5] C. Stosiek, O. Garaschuk, K. Holthoff, and A. Konnerth, "In vivo two-photon calcium imaging of neuronal networks", Proc. Natl. Acad. Sci. U.S.A. 100, 7319-7324 (2003). 
[6] J. Pawley, Handbook of biological confocal microscopy (Springer) (1990).

[7] J. A. Izatt, M. R. Hee, G. M. Owen, E. A. Swanson, and J. G. Fujimoto, "Optical coherence microscopy in scattering media”, Opt. Lett. 19, 590-592 (1994).

[8] J. Yao and L. V. Wang, "Photoacoustic microscopy", Laser Photonics Rev. 7, 758-778 (2013).

[9] W. Denk, J. H. Strickler, and W. W. Webb, “Two-photon laser scanning fluorescence microscopy", Science 248, 73-76 (1990).

[10] A. Badon, A. C. Boccara, G. Lerosey, M. Fink, and A. Aubry, "Multiple scattering limit in optical microscopy”, Opt. Express 25, 28914-28934 (2017).

[11] L. V. Wang and H.-I. Wu, Biomedical optics: principles and imaging (John Wiley \& Sons) (2009).

[12] S. L. Jacques, "Optical properties of biological tissues: a review”, Phys. Med. Biol. 58, R37-R61 (2013).

[13] J. Gómez Rivas, R. Sprik, C. M. Soukoulis, K. Bush, and A. Lagendijk, "Optical transmission through strong scattering and highly polydisperse media”, EPL 48, 2228 (1999).

[14] F. Martelli, T. Binzoni, A. Pifferi, L. Spinelli, A. Farina, and A. Torricelli, "There's plenty of light at the bottom: statistics of photon penetration depth in random media", Sci. Rep. 6, 1-14 (2016).

[15] M. Fink, D. Cassereau, A. Derode, C. Prada, P. Roux, M. Tanter, J.-L. Thomas, and F. Wu, "Time-reversed acoustics", Rep. Prog. Phys. 63, 1933-1995 (2000).

[16] G. Lerosey, J. de Rosny, A. Tourin, A. Derode, G. Montaldo, and M. Fink, "Time reversal of electromagnetic waves", Phys. Rev. Lett. 92, 193904 (2004).

[17] I. M. Vellekoop and A. P. Mosk, "Focusing coherent light through opaque strongly scattering media", Opt. Lett. 32, 2309-2311 (2007).

[18] S. M. Popoff, G. Lerosey, R. Carminati, M. Fink, A. C. Boccara, and S. Gigan, "Measuring the transmission matrix in optics: An approach to the study and control of light propagation in disordered media”, Phys. Rev. Lett. 104, 100601 (2010).

[19] A. P. Mosk, A. Lagendijk, G. Lerosey, and M. Fink, "Controlling waves in space and time for imaging and focusing in complex media", Nat. Photonics 6, 283-292 (2012).

[20] S. Rotter and S. Gigan, "Light fields in complex media: Mesoscopic scattering meets wave control”, Rev. Mod. Phys. 89, 015005 (2017).

[21] R. K. Tyson, Principles of Adaptive optics (CRC Press) (2015).

[22] J. Kubby, S. Gigan, and M. Cui, Wavefront Shaping for Biomedical Imaging, Advances in Microscopy and Microanalysis (Cambridge University Press) (2019). 
[23] R. Horstmeyer, H. Ruan, and C. Yang, "Guidestar-assisted wavefront-shaping methods for focusing light into biological tissue”, Nat. Photonics 9, 563-571 (2015).

[24] M. J. Booth, "Adaptive optical microscopy: the ongoing quest for a perfect image", Light Sci. Appl. 3, e165 (2014).

[25] N. Ji, “Adaptive optical fluorescence microscopy”, Nat. Methods 14, 374-380 (2017).

[26] S. Yoon, M. Kim, M. Jang, Y. Choi, W. Choi, S. Kang, and W. Choi, "Deep optical imaging within complex scattering media", Nat. Rev. Phys. 2, 141-158 (2020).

[27] S. Gigan, “Optical microscopy aims deep”, Nat. Photonics 11, 14-16 (2017). 


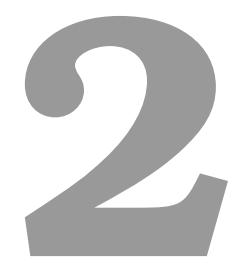

\section{Blind focusing through strongly scattering media using wavefront shaping with nonlinear feedback}

Scattering prevents light from being focused in turbid media. The effect of scattering can be negated through wavefront shaping techniques when a localized form of feedback is available. Even in the absence of such a localized reporter, wavefront shaping can blindly form a single diffraction-limited focus when the feedback response is nonlinear. We developed and experimentally validated a model that accurately describes the statistics of this blind focusing process. We show that maximizing the nonlinear feedback signal only results in the formation of a focus when a limited number of reporters are contributing to the signal. Using our model, we can calculate the minimal requirements for the number of controlled spatial light modulator segments and the order of nonlinearity to blindly focus light through strongly scattering media.

\subsection{Introduction}

Refractive index inhomogeneities in a turbid medium scatter light in a complex manner. Consequently, a focus inside these types of media becomes more aberrated with increasing depth, until ultimately no ballistic light is left and the focus decays into a random speckle pattern. Even at this depth, light can still be focused through wavefront shaping techniques $[1,2]$. These techniques have been used to focus light inside scattering media for various applications, such as optical manipulation [3], optogenetics [4] and fluorescence microscopy through

This chapter has been published as: [G. Osnabrugge, L.V. Amitonova and I.M. Vellekoop, Opt. Express 27, 11673-11688 (2019)]. 


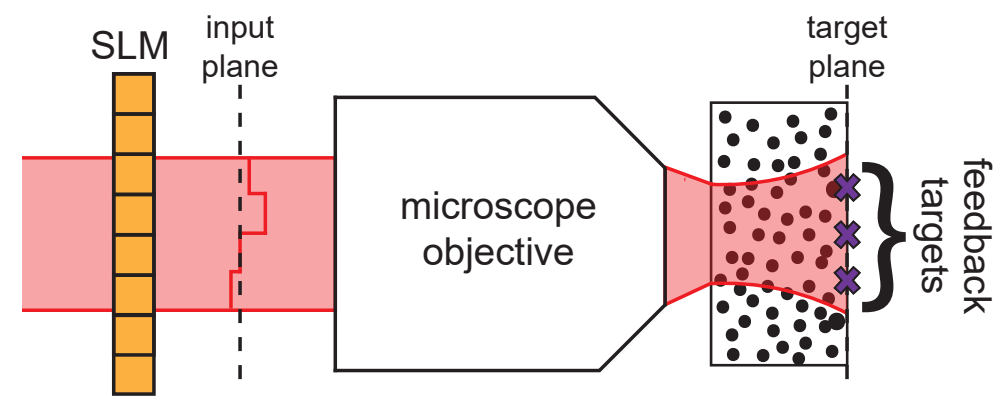

Figure 2.1: Illustration of the blind focusing experiment. SLM: Spatial light modulator.

an intact skull of a mouse [5]. Using wavefront shaping techniques, light has been focused through several centimeters of biological tissue [6].

The main limitation of these techniques is the need for a localized reporter (e.g. guide star), providing feedback of the light intensity at the focus location. Many different mechanisms can act as localized reporters, such as point detectors, fluorescent or nonlinear markers, acoustically tagged light and photoacoustic absorbers [2]. However, a localized reporter is not always available. For instance in multiphoton fluorescence excitation microscopy, generally all structures of interest are stained with fluorescent markers, which will all generate a signal when illuminated. When a weak ballistic unscattered component is still preserved, wavefront shaping with this mixed form of feedback can be used to correct focus aberrations [7-9].

Katz et al. [10] showed that even in the absence of a localized reporter and a ballistic component, light can be focused through a strongly scattering layer. In their experiment, the nonlinear feedback signal was generated by many indistinguishable fluorescent sources hidden behind the opaque layer. Maximizing the total nonlinear feedback signal using wavefront shaping resulted in the formation of a single diffraction-limited focus. We term this technique 'blind focusing' since the scattered light is spatially focused without the need for any information about the location of the sources or about the scattered light distribution. In the case of a pulsed light source, blind focusing is not limited to spatial focusing only, but can also result in a temporally compressed focus $[11,12]$. Therefore, blind focusing is potentially a powerful technique for nonlinear deep-tissue microscopy. So far, a theoretical understanding of this iterative optimization process has been missing, and, therefore, it is not known under which conditions the blind focusing method will converge to a single diffraction-limited focus.

Here, we present and validate a model that accurately describes the statistics of blind focusing. Using this model, we show that focusing is only possible when certain minimal requirements are met. Additionally, we are able to predict the evolution of the optimized speckle pattern during the optimization process.

We will first derive the exact solution of the scattered field behind a strongly scattering layer for a single (known) realization of the scattering medium. Afterwards, we formulate a statistical model that describes the probability density function of the light intensity, averaged over the ensemble of possible samples. Our predictions are validated in a set of experiments with first, second and third order feedback. 


\subsection{Theory of blind focusing}

A simplified illustration of the blind focusing experiment is shown in Fig. 2.1. The goal of this experiment is to form a single diffraction limited focus through a scattering layer at the target plane using nonlinear feedback from $M$ targets. A spatial light modulator (SLM) is used to control the field $E_{a}$ at the input plane. The feedback signals from the individual targets cannot be distinguished and only the total generated signal is recorded. We define this feedback signal as

$$
S \equiv \sum_{b}^{M}\left|E_{b}\right|^{2 n}
$$

where $E_{b}$ is the electric field at target $b$ and $n$ is the order of feedback. For instance, in fluorescence imaging, $n=2$ corresponds to two-photon excitation microscopy.

We start the experiment by constructing an arbitrary field using the SLM. Afterwards, we perform the stepwise sequential wavefront shaping algorithm [1] to optimize the feedback signal $S$. In contrast to the genetic wavefront shaping algorithm [13] used in previous work by Katz et al. [10], our method is deterministic and can be analyzed analytically. Each iteration of the algorithm results in an optimized incident wavefront, which is used as the starting wavefront of the next iteration of the algorithm. This process is repeated until the algorithm has reached a fixed point, where the optimized wavefront does not change anymore. In Appendix 2.7.1, we prove that such a fixed point always corresponds to a (local) maximum of $S$.

In this section, we will derive how the target field $E_{b}$ changes as the $n$ th-order feedback signal $S$ is optimized by iteratively running the algorithm. Although $S$ is nonlinear, we can describe the light propagation to the target plane as a linear combination of input fields

$$
E_{b}^{(k)}=\sum_{a}^{N} t_{b a} E_{a}^{(k)}
$$

Here, $t_{b a}$ is an element of the transmission matrix $\mathbf{T}, N$ is the number of independently controlled segments on the SLM and the superscript number $k$ between brackets indicates the iteration number. We take the total incident intensity $\sum_{a}^{N}\left|E_{a}\right|^{2}=1$.

For the $(k+1)$ th wavefront shaping iteration, we use the optimized wavefront from the previous iteration as our new starting wavefront. Then we shift the phase of a single incident field segment a', such that $E_{a^{\prime}}^{(k)} \rightarrow E_{a^{\prime}}^{(k)}+E_{a^{\prime}}^{(k)}\left[e^{i \phi}-1\right]$. We measure $S$ as we vary $\phi$ from 0 to $2 \pi$ in $P$ steps. Using Eq. (2.1) and Eq. (2.2), the intermediary feedback signal can now be written as

$$
\tilde{S}^{(k+1)}(\phi)=\sum_{b}^{M}\left(\left|E_{b}^{(k)}\right|^{2}+\left|t_{b a^{\prime}} E_{a^{\prime}}^{(k)}\right|^{2}\left|e^{i \phi}-1\right|^{2}+\left(E_{b}^{(k)} t_{b a^{\prime}}^{*} E_{a^{\prime}}^{(k) *}\left[e^{-i \phi}-1\right]+\text { c.c. }\right)\right)^{n},
$$

where ${ }^{*}$ represents the complex conjugate. The effect of the perturbation $E_{a^{\prime}}^{(k)}\left[e^{i \phi}-1\right]$ on the feedback signal is expected to be small. Therefore, we expand $\tilde{S}^{(k+1)}$ in terms of the perturbation to arrive at

$$
\tilde{S}^{(k+1)}(\phi) \approx S^{(k)}+\sum_{b}^{M}\left(W_{b}^{(k)} t_{b a^{\prime}}^{*} E_{a^{\prime}}^{(k) *}\left[e^{-i \phi}-1\right]+c . c .\right),
$$


with the nonlinearly weighted target field $W_{b}^{(k)} \equiv n\left|E_{b}^{(k)}\right|^{2(n-1)} E_{b}^{(k)}$ (see Appendix 2.7.1 for a rigorous mathematical treatment).

Following the processing steps as described in Ref. [14], we then find the optimized wavefront by isolating the contribution of $\phi$ using the following expression

$$
E_{a^{\prime}}^{(k+1)}=\frac{c^{(k+1)}}{E_{a^{\prime}}^{(k) *} P} \sum_{p}^{P} \tilde{S}^{(k+1)}\left(\phi_{p}\right) e^{i \phi_{p}}=c^{(k+1)} \sum_{b}^{M} W_{b}^{(k)} t_{b a^{\prime}}^{*},
$$

where $c^{(k+1)}$ is a normalization factor, which normalizes the total incident intensity to 1 . The phase shift $\phi$ at segment $a^{\prime}$ is set back to 0 and the process is then repeated for all other SLM segments. Finally, by inserting Eq. (2.5) into Eq. (2.2), we arrive at an expression for the resulting target field after the $(k+1)$ th wavefront shaping iteration

$$
E_{b}^{(k+1)}=c^{(k+1)} \sum_{a}^{N} \sum_{b^{\prime}}^{M} t_{b a} t_{b^{\prime} a}^{*} W_{b^{\prime}}^{(k)} .
$$

We recognize that the optimized target field in Eq. (2.6) is the nonlinearly weighted sum over the phase conjugated fields propagated from the target locations. The nonlinear weighted field scales with the field strength to the power $2 n-1$, and thus the brighter targets will have a stronger contribution to the optimized incident field. Performing multiple iterations of wavefront can therefore ultimately result in a focus being formed at one of the targets.

The expression in Eq. (2.6) can be connected to previous iterative wavefront shaping experiments. For linear feedback, $W_{b}^{(k)}$ reduces to $E_{b}^{(k)}$. In this case, after performing the wavefront shaping algorithm multiple times, $E_{b}^{(k)}$ will converge to the eigenvector of matrix $\mathbf{T}$ with the highest eigenvalue [15]. In other words, instead of forming a focus, the algorithm will instead optimize the total transmission through the scattering sample. Alternatively, for $n>1$ in a weakly scattering medium, where $\mathbf{T}$ is close to unitary, the optimized target field becomes $E_{b}^{(k+1)} \propto\left|E_{b}^{(k)}\right|^{2(n-1)} E_{b}^{(k)}$. Now the brightest targets are enhanced more than the dimmer targets until finally a focus is formed at the brightest target [16]. However, we will show that when $\mathbf{T}$ is not close to unitary, as is the case in strongly scattering media, maximizing $S$ is not necessary equivalent to forming a focus.

\subsection{Statistical model for blind focusing}

Next, we want calculate the optimized target intensity distribution through a strongly scattering medium with a non-unitary transmission matrix. Finding the exact value of the target field, using Eq. (2.6), requires full knowledge of the transmission matrix, which often cannot be obtained. Therefore, we will instead calculate the probability density function of $E_{b}^{(k+1)}$, averaged over the ensemble of possible samples. We assume that $W_{b}^{(k)}$ is known and independent of $\mathbf{T}$. The transmission matrix is assumed to be a random matrix with independent elements, such that the expectation value $\left\langle t_{b a} t_{b^{\prime} a^{\prime}}^{*}\right\rangle=\delta_{b^{\prime} b} \delta_{a^{\prime} a}\left\langle\left|t_{b a}\right|^{2}\right\rangle$, where $\delta$ is the Kronecker delta. In Appendix 2.7.2, we derive that for an imperfect wavefront shaping setup the probability density function of $E_{b}^{(k+1)}$ is a complex normal distribution of the form:

$$
P\left(E_{b}^{(k+1)}\right)=\frac{1}{\pi I_{0}} \exp \left(-\frac{\left|E_{b}^{(k+1)}-\mu_{b}^{(k+1)}\right|^{2}}{I_{0}}\right) .
$$


Here, the average optimized field is given by

$$
\mu_{b}^{(k+1)} \equiv \frac{W_{b}^{(k)}}{\sqrt{\sum_{b^{\prime}}^{M}\left|W_{b^{\prime}}^{(k)}\right|^{2}}} \gamma \sqrt{N I_{0}},
$$

with $I_{0} \equiv\left\langle\left|t_{b a}\right|^{2}\right\rangle$. The quality of the wavefront modulation is described by the fidelity parameter $|\gamma|^{2}$. This parameter's value ranges between 0 and 1, where a value of 1 corresponds with a perfect wavefront modulation. Unlike regular speckle fields, $E_{b}^{(k+1)}$ will have a non-zero average value $\mu_{b}^{(k+1)}$ because of the performed wavefront shaping iteration.

When $E_{b}^{(k+1)}$ follows a complex normal distribution with a non-zero mean, the corresponding optimized target intensity $I_{b}^{(k+1)} \equiv\left|E_{b}^{(k+1)}\right|^{2}$ follows a modified Rice distribution [17]. For $\mu_{b}^{(k)}=0$, this probability density function reduces to an exponential distribution as normally seen in speckle statistics. For now, we are mainly interested in the average optimized intensity and the corresponding standard deviation at target $b$, which are given by

$$
\left\langle I_{b}^{(k+1)}\right\rangle=\left|\mu_{b}^{(k+1)}\right|^{2}+I_{0}=I_{0}\left(N|\gamma|^{2} \frac{\left|W_{b}^{(k)}\right|^{2}}{\sum_{b^{\prime}}^{M}\left|W_{b^{\prime}}^{(k)}\right|^{2}}+1\right)
$$

and

$$
\sigma_{I}=\sqrt{I_{0}\left|\mu_{b}^{(k+1)}\right|^{2}+I_{0}^{2}}=I_{0} \sqrt{N|\gamma|^{2} \frac{\left|W_{b}^{(k)}\right|^{2}}{\sum_{b^{\prime}}^{M}\left|W_{b^{\prime}}^{(k)}\right|^{2}}+1}
$$

respectively. Note that $\left\langle I_{b}^{(k+1)}\right\rangle$ increases with $N$, whereas $\sigma_{I}$ increases with $\sqrt{N}$.

After the $(k+1)$ th wavefront shaping iteration, the average intensity at target $b$ depends on the nonlinearly weighted fields at all targets. The targets which are generating a strong signal will contribute more to the feedback signal $S$, and will therefore, on average, be enhanced more than the weaker targets. We recognize that if nearly all of the feedback signal is coming from a single target, then the average intensity enhancement can be approximated by $\langle\eta\rangle \equiv$ $\left\langle I_{b}^{(k+1)}\right\rangle / I_{0} \approx N|\gamma|^{2}+1$. This expression is equivalent to the enhancement in a conventional single target wavefront shaping experiment [14]. However, when a large number of targets are contributing to the feedback signal, the expected enhancement is reduced to 1 , and as a result, no focus is formed at all.

To summarize, during the blind focusing experiment, we attempt to focus light through a strongly scattering sample using wavefront shaping. In this experiment, multiple targets contribute to the total feedback signal. We developed a statistical model that predicts how much the light intensity at each feedback targets will be optimized. The average value and standard deviation of the optimized target intensities are given by Eq. (2.9) and Eq. (2.10), respectively. This model can be used to analyze the evolution of target intensity distribution during the blind focusing process. 


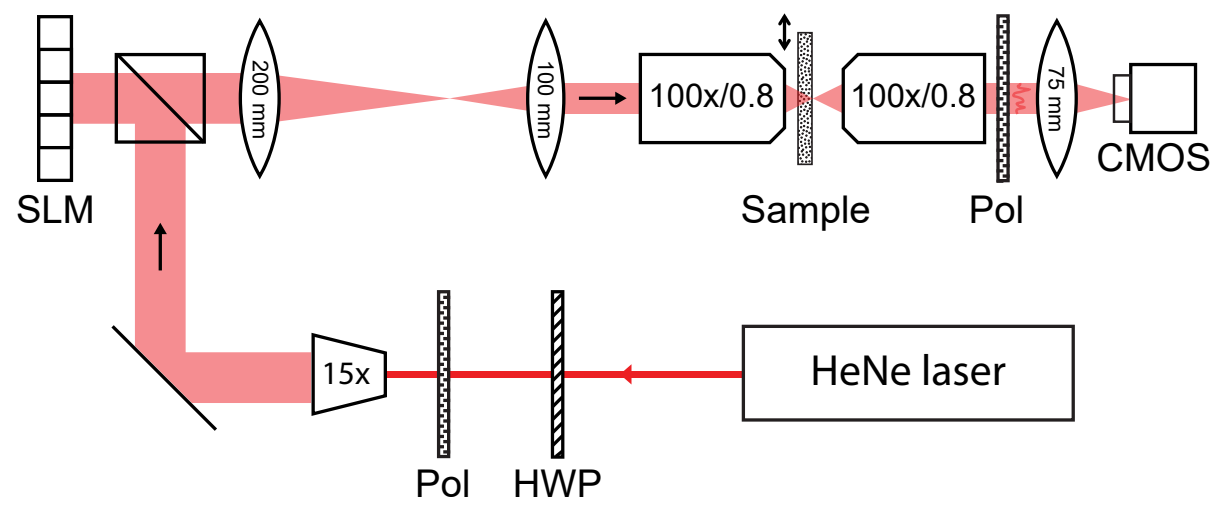

Figure 2.2: Schematic of the experimental setup. HWP: half wave plate, Pol: Polarizer, SLM: Spatial light modulator, CMOS: Complementary metal oxide semiconductor camera.

\subsection{Experimental validation}

\subsubsection{Experimental setup}

We will now experimentally validate the prediction for the optimized intensity as given by Eq. (2.9). Our experimental setup is illustrated in Fig. 2.2. Light from a HeNe laser is expanded and modulated by a phase-only spatial light modulator (Hamamatsu X13138-07). With two lenses in a 4f-configuration, the SLM-modulated wavefront is conjugated to the back focal plane of a microscope objective (Zeiss A-Plan 100x/0.8), which focuses the light onto the surface of a scattering sample. As our source of feedback, we chose to use a CMOS camera (Basler acA640-750um), which measures the intensity distribution at the back surface of the scattering sample through an identical microscope objective. On the camera, we set groups of $3 \times 3$ pixels (corresponding to $0.16 \times 0.16 \mu \mathrm{m}^{2}$ ) as independent targets for the wavefront shaping algorithm. Using this experimental setup, we can choose the order of feedback, and we can accurately set the number of targets contributing to the feedback signal. We would like to emphasize that in these experiments only the total $n$-th order intensities summed over all targets was used as the feedback signal, mimicking the nonlinear response of fluorescent markers in a multiphoton fluorescence excitation microscope.

As a scattering sample, we used a glass substrate dip-coated in a suspension of $5 \%$ zincoxide (Sigma Aldrich, average grain size $200 \mathrm{~nm}$ ) and demineralized water. The thickness of the layer were measured to be $37.6 \pm 9.8 \mu \mathrm{m}$. Based on previous work [18], the transport mean free path of the zinc-oxide sample is expected to be around $0.6 \mu \mathrm{m}$, ensuring that all light passing through the sample is multiple scattered. The sample is mounted on a translation stage (Zaber T-LSM050A), to allow the interrogation of different sample locations for statistical averaging. The average wavefront shaping fidelity of our setup was measured to be $\left\langle|\gamma|^{2}\right\rangle=0.39$ with $N=208$.

\subsubsection{Blind focusing experiment with two feedback targets}

We performed a blind focusing experiment, where two targets were simultaneously optimized using the wavefront shaping algorithm as described before. The targets are separated by a 
distance $4.4 \mu \mathrm{m}$. On the SLM, a random pattern of $N=208$ square segments was displayed, matching the size of the light beam on the SLM. The experiment was performed 100 times, changing the initial SLM pattern and sample lateral position in between every experiment. Examples of the intensity distributions measured on the back side of the sample, before and after the optimization, are shown in Fig. 2.3a and b, respectively. Here, targets 1 and 2 are indicated by the red (right) and blue (left) circles. In these figures, the starting intensity of target 2 is much higher than the intensity target 1 . As a result, the contribution of target 2 to the feedback signal is much larger than target 1 , and therefore, only the intensity of target 2 is enhanced. Fig. 2.3c-e show the optimized intensities $I^{(1)}$ of target 1 (red circles) and target 2 (blue squares) as function of the ratio of the initial intensities $I^{(0)}$ of the 2 targets, for firstorder, second-order and third-order feedback. All intensities are normalized to $I_{\max }=N|\gamma|^{2} I_{0}$, which is the average optimized intensity obtained by performing a single-target wavefront shaping experiment. The solid lines and the shaded areas indicate the average optimized intensities and the standard deviation as predicted by Eq. (2.9) and Eq. (2.10).

In Fig. 2.3c-e, we see that even when both targets contribute to the feedback signal, the target intensities are not necessarily equally enhanced. Neither are the optimized intensities $I^{(1)}$ randomly distributed, but rather the optimized intensities are directly related to the starting intensities of target 1 and 2, as expected from Eq. (2.9). For $n=1$, the optimized intensities at the targets are linearly proportional to the initial intensities, since in that case $\left|W_{b}^{(0)}\right|^{2}$ reduces to $I_{b}^{(0)}$. For higher-order feedback measurements, the relation between the initial intensity ratio and the optimized intensity becomes nonlinear. As a result, a small difference in $I^{(0)}$ between the two targets can result in a big difference between optimized intensities. For instance for $n=3$, only approximately $65 \%$ of the total initial intensity is required in one target to ensure that, in most experiments, only the intensity of that target is optimized. This thresholding effect for $n>1$, observed in Fig. $2.3 \mathrm{~d}$ and e, is the mechanism that allows the blind focusing method to form a single diffraction-limited focus even when multiple targets contribute to the feedback signal. The data is in good agreement with our theoretical predictions and most of the data points fall within the statistical variation as described in Eq. (2.10). Other deviations might be explained by fluctuations in the fidelity during the experiment.

\subsection{Blind focusing requirements}

\subsubsection{Minimal requirements}

Now, we want to use our statistical model to find out under which circumstances the blind focusing method is able to form a single focus, when $M$ targets are contributing to the feedback signal. Instead of analyzing convergence behaviour starting from a random speckle pattern, we analyze the case where light is already focused to one target before the optimization. The focus should be preserved after the wavefront shaping iteration when this focus corresponds to a fixed point of the algorithm. To quantify the intensity in the focus, we use the enhancement, which is defined as $\eta \equiv I / I_{0}$. The starting enhancement in the focus is given by $\eta^{(0)}$. Furthermore, we assume that all other $(M-1)$ targets are exponentially distributed with an average starting enhancement of 1. Inserting these parameters into Eq. (2.9) produces an expression for the expected optimized focus enhancement after a single wavefront shaping 

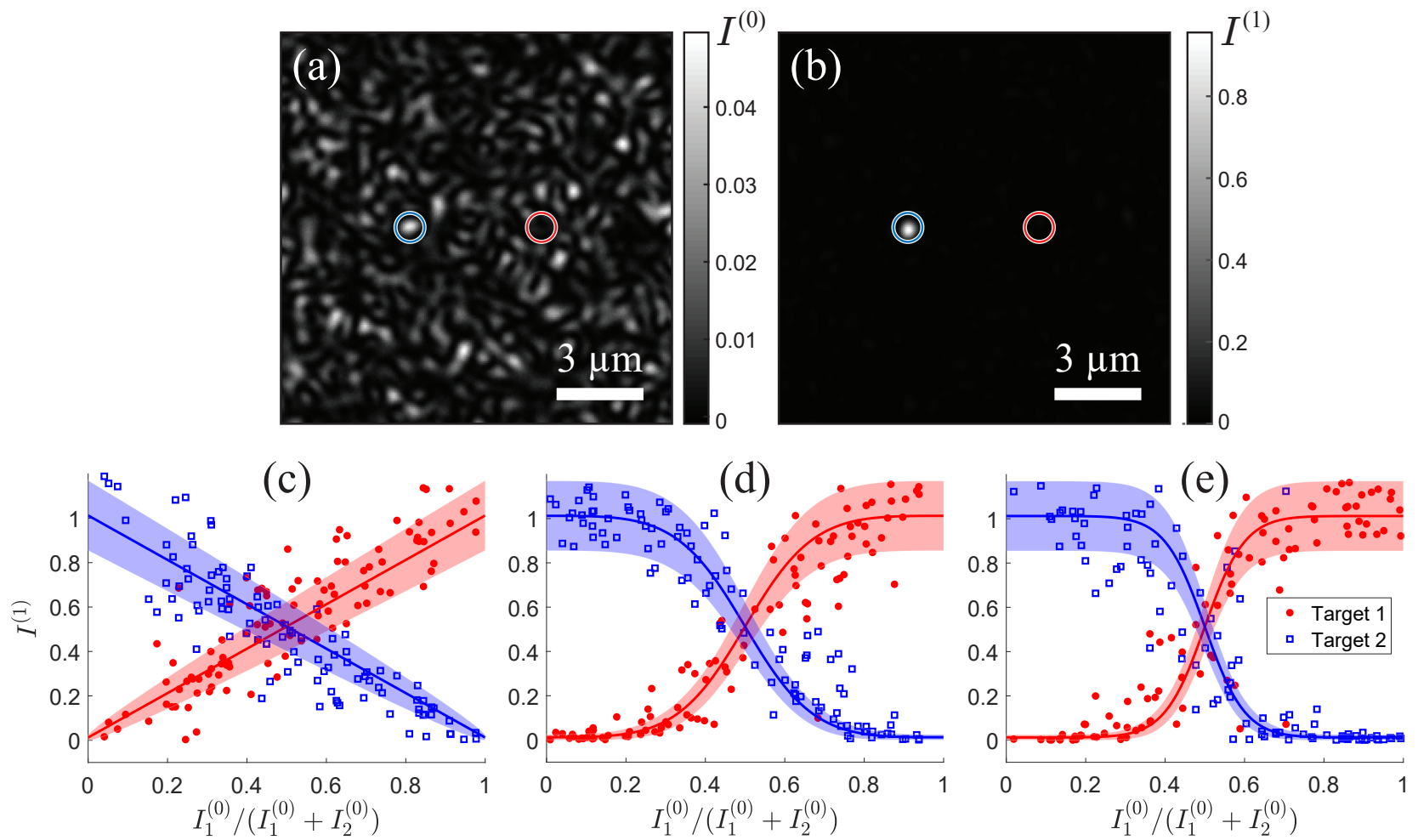

Figure 2.3: Results of the blind focusing experiment using two feedback targets. (a) Example image of the initial intensity distribution $\left(I^{(0)}\right)$, and (b) the corresponding intensity distribution after wavefront shaping $\left(I^{(1)}\right)$. The red (right) and blue (left) circles mark the locations of targets 1 and 2. (c)-(e) The optimized target intensities plotted as function of the ratio of target intensities before the optimization. The optimized intensities at target 1 and 2 are represented by the red circles and blue squares, respectively. The experiments were performed using (c) first-order, (d) second-order, and (e) third-order feedback. All intensities are normalized to $I_{\max }=N|\gamma|^{2} I_{0}$. The predicted mean value for the optimized intensity and the corresponding standard deviation, as given by Eq. (2.9) and Eq. (2.10), are represented by the colored solid lines and the shaded areas. 
iteration

$$
\left\langle\eta^{(1)}\right\rangle=N|\gamma|^{2} \frac{\left(\eta^{(0)}\right)^{2 n-1}}{\left(\eta^{(0)}\right)^{2 n-1}+(2 n-1) !(M-1)}+1 .
$$

We recognize that $\left\langle\eta^{(1)}\right\rangle$ can be smaller than $\eta^{(0)}$ for a large $M$. In order for the blind focusing method to be able to form a focus, the average focus enhancement after the optimization should be equal or larger to the enhancement before the optimization. In Appendix 2.7.3, we show that (given $N,|\gamma|^{2}$ and $n$ ) an upper limit for the number of feedback targets can derived, which is given by

$$
M_{\max }=\frac{(2 n-2)^{2 n-2}}{(2 n-1) !(2 n-1)^{2 n-1}}\left(|\gamma|^{2} N\right)^{2 n-1}+1 .
$$

Whenever $M>M_{\max }$, a focus can, on average, not be formed. Rather, the wavefront shaping algorithm optimizes the intensity and contrast of the full speckle pattern to maximize the feedback signal. We see that the order of feedback $n$ can be increased to guarantee blind focusing convergence for a larger number of contributing targets. Moreover, by increasing $N$ by a factor of $\alpha, M_{\max }$ increases by a factor of $\alpha^{2 n-1}$.

\subsubsection{Blind focusing experiment with multiple targets}

To verify the prediction in Eq. (2.11), we performed a second set of experiments using large feedback areas containing $M$ feedback targets. For this experiment, we used the same experimental setup as described before. We start our experiment with a pre-optimized focus at a single target, whereas the remaining targets in the feedback area are illuminated by a random speckle pattern. The pre-optimized focus is obtained by first optimizing for a single target in the center of the camera frame. Afterwards, a wavefront shaping iteration is performed using the total second-order feedback signal from all targets within a small and a large region of interest (ROI), which have a radius of $2.1 \mu \mathrm{m}$ and $10.5 \mu \mathrm{m}$. Based on the average speckle size, the number of targets are estimated to be $M=96$ and $M=2400$ for the small and the large ROIs, respectively. The blind focusing experiments were performed for $N=80$ and $N=208$, and were performed 100 times for both ROIs. In between each experiment, the lateral sample position was changed and the intensity of pre-optimized focus was varied by adding a controlled amount of uniformly-distributed noise to the starting wavefront.

In Fig. 2.4a, an example image of a starting speckle pattern with a pre-optimized focus is shown. The red and purple circles in the Fig. indicate the size of the small and large ROIs. In Fig. 2.4b and c, the enhancement of the pre-optimized focus intensity after the blind focusing experiment $\eta^{(1)}$ is plotted as function of the starting enhancement of the preoptimized focus, for $N=80$ (Fig. 2.4b) and $N=208$ (Fig. 2.4c). The experiments using the small and the large ROI for feedback are represented by the red circles and the purple squares, respectively. In Fig. 2.4b and c, the expected value for $\eta^{(1)}$ (as predicted by Eq. (2.11)) and the corresponding standard deviation are represented by the colored solid lines and the shaded areas, respectively. The black solid lines indicates the identity lines, where $\eta^{(1)}=\eta^{(0)}$.

In Fig. 2.4b and c, two general regions can be recognized. The data points above the identity line represent experiments where the focus enhancement increased, whereas the data points under the identity line represent a decrease in the focus enhancement. In Fig. 2.4b, in the experiments with $M=96$ and $N=80$, nearly all measured $\eta^{(1)}$ values lie above the 


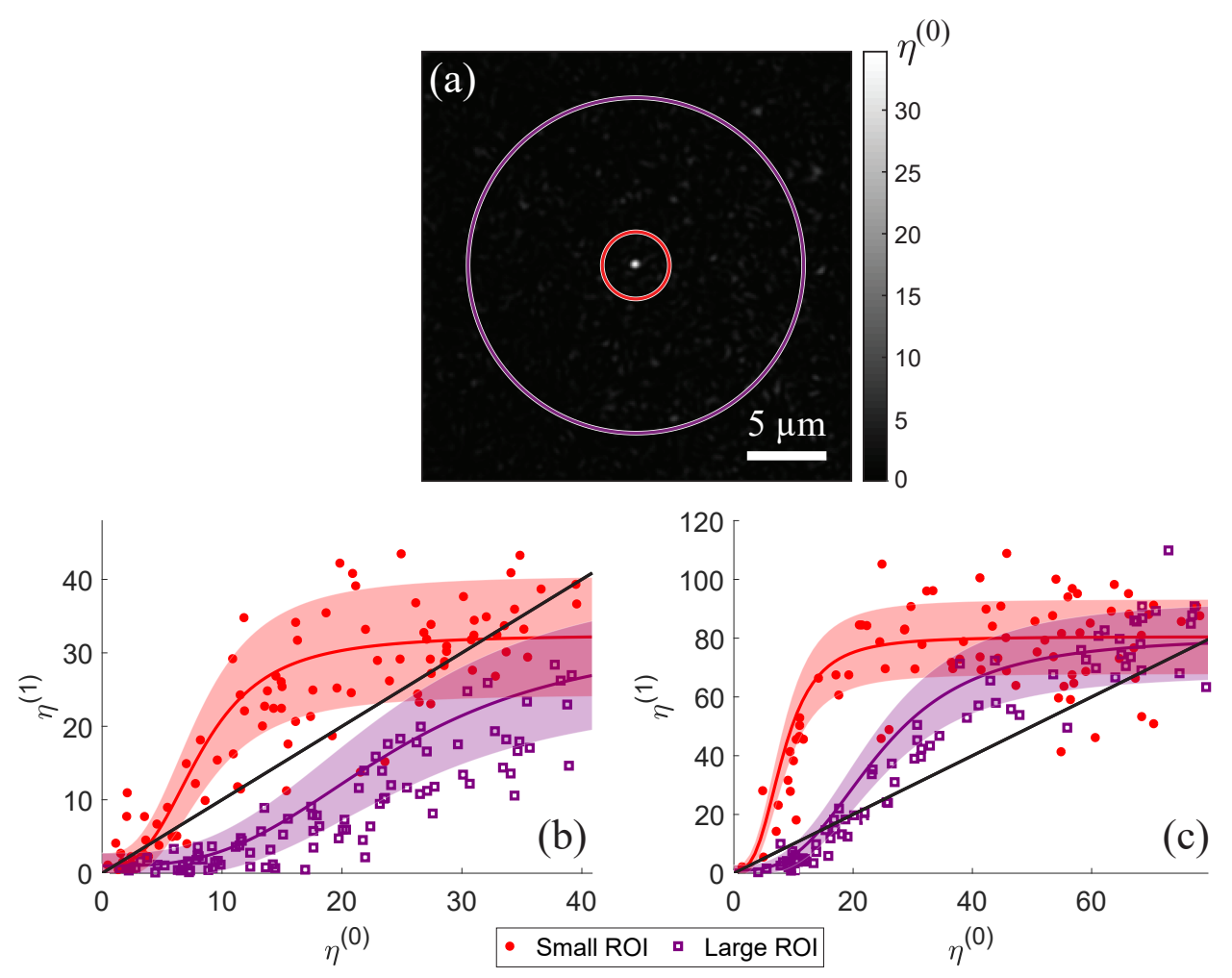

Figure 2.4: Results of the blind focusing experiments with a pre-optimized focus using the total second-order feedback signal from a region of interest containing $M$ targets. (a) Example speckle pattern with a pre-optimized focus, where the red and purple circles represent the small $(M=96)$ and large $(M=2400)$ ROIs, respectively. (b)-(c) The enhancement $\eta$ in the pre-optimized focus before and after blind focusing with $M=96$ (red circles) and $M=2400$ (purple squares), with (b) $N=80$, and (c) $N=208$. The predicted mean value for $\eta^{(1)}$ and the corresponding standard deviation are represented by the colored solid lines and the shaded areas, respectively. The black solid line indicates the identity line. 
identity line, meaning that the focus enhancement increases after the iteration of wavefront shaping. However, in the experiments with the large ROI, $\eta^{(1)}$ is almost always lower than $\eta^{(0)}$. These results suggest that the large ROI contains too many competing targets for the pre-optimized focus to be preserved. In other words, the wavefront shaping algorithm favors optimizing the intensity of multiple targets within the ROI over preserving the intensity in the pre-optimized focus.

In Fig. 2.4c, the number of controlled SLM segments was increased to $N=208$. Here, the $\left\langle\eta^{(1)}\right\rangle$ curves cross the identity curves for both the small and large ROIs, indicating that the intensity in the pre-optimized focus is was further enhanced. Increasing $N$ thus allows blind focusing to preserve a focus for a higher number of targets that are contributing to the feedback signal. The experimental data is in excellent agreement with the prediction in Eq. (2.11).

\subsubsection{Breakdown of blind focusing}

Finally, we performed experiments to test whether a pre-optimized focus is preserved over multiple wavefront shaping iterations when the number of targets exceeds the blind focusing upper limit, given in Eq. (2.12). The feedback signal was obtained using the same small and large ROIs as described before, with second-order feedback and $N=80$. The algorithm was performed for 10 iterations, where for each iteration, the optimized wavefront of the preceding iteration was used as the new starting wavefront. The resulting speckle images and the corresponding feedback signals of these experiments are included in the supplementary materials* as Visualization 1 (small ROI) and Visualization 2 (large ROI).

In all the experiments using the small feedback ROI, the initial pre-optimized focus was preserved over all 10 iteration as the feedback signal was enhanced. However, in the large ROI experiments, the pre-optimized focus decays into a speckle pattern after only running the algorithm for 2 to 3 optimization iterations. Here, the feedback signal clearly increased during the optimization even though the starting focus was lost in the process. These results demonstrate that for $n=2$ and $N=80$, the blind focusing method is not be able to form a focus. This is in agreement with the prediction in Eq. (2.12), since the number of feedback targets in the large ROI $(M=2400)$ exceeded the upper limit, $M_{\max }=751$. As a reference, the succesful blind focusing experiments with $N=208$ and $n=2$ (shown in Fig. 2.4c) have a much higher $M_{\max }$ of 13181 targets.

\subsection{Discussion}

We studied wavefront shaping with nonlinear feedback through a strongly scattering sample using the stepwise sequential algorithm [14]. This deterministic algorithm allowed us to derive the exact solution for the optimized field at the feedback targets, which is given in Eq. (2.6). This expression shows that in weakly scattering samples, the optimization algorithm takes higher orders of the initial target field with every iteration. As a result, only the brightest speckles at the target plane will be enhanced [16]. Therefore, wavefront shaping and adaptive optics techniques can be used to improve the intensity of the focus in multiphoton fluorescence excitation microscopy without the need for a guide star [7, 8].

*See: https://www.osapublishing.org/oe/abstract.cfm?uri=oe-27-8-11673\#articleSupplMat 
Previous work demonstrated that a focus can be formed through a strongly scattering layer without the need for a localized reporter [10]. We showed that in strongly scattering samples, the statistics of the optimized target intensities are accurately described by the Rice distribution. However, due to the large standard deviation in the distribution, it remains hard to predict at which target the focus will be formed when the targets are illuminated with a random speckle pattern.

In Appendix 2.7.1, we showed that a fixed point of our optimization algorithm always corresponds to a maximum of the feedback signal. Moreover, we experimentally demonstrated that when the number of targets exceeds $M_{\max }$ from Eq. (2.12), our algorithm is, on average, unable to form a focus. As a result, maximizing $S$ does not always guarantee the formation of a focus, but will instead optimize the intensity and contrast of the speckle pattern. This limitation applies to all optimization algorithms using the total nonlinear signal as feedback, including the genetic wavefront shaping algorithm [13].

In our experiments, we only considered targets distributed across a flat image plane. However, our model can also be employed to point targets on an arbitrarily shaped plane. Therefore, our model can easily be generalized for scattering samples with feedback targets distributed in all three spatial dimensions. In such samples, the light intensity will spread out more, the deeper the light propagates into the sample. As a result, the number of illuminated targets will also rapidly increase with focusing depth. In order for the blind focusing requirements to be fulfilled, the labeling density of these samples might need to be restricted.

In our statistical model, we assumed that the nonlinearly weighted field $W_{b}^{(k)}$ is independent of the transmission matrix. As a result, we ignore certain properties of the matrix $\mathbf{T}$ known from random matrix theory, such as the distribution of transmission eigenvalues [19]. Nevertheless, our statistical model is in a surprisingly good agreement with the experimental data. At the moment, our model is unable to predict the rate of convergence. Therefore, we believe that studying these properties of random matrices will be interesting to obtain a full understanding of this nonlinear optimization process.

\subsection{Appendices}

\subsubsection{Convergence to local maximum}

We used the stepwise sequential optimization algorithm [1] in an attempt to maximize the total feedback signal $S$. For linear feedback from a single target, it is well known that this algorithm finds a global maximum for the intensity at that target in a single iteration [14]. In the case of nonlinear feedback originating from multiple targets, however, the optimization problem has multiple local maxima. In this situation, it is not directly trivial that the algorithm finds a local maximum of $S$; it is not even directly clear that the stepwise sequential algorithm increases $S$ at all.

Here, we will prove that all local maxima of $S$ correspond to attractive fixed points of the algorithm (Eq. (2.6)) and vice versa. Consequentially, when the algorithm converges, it converges to a local maximum of $S$ as well. Furthermore, this proof implies that each local maximum of $S$ has a finite region of attraction for which the algorithm will converge to that local maximum. Note that we cannot exclude the existence of initial conditions for which the algorithm does not converge. However, we have not observed these cases in our experiments. 
In order to simplify the derivations, we introduce a compact vector notation, replacing $E_{a}$ by $\mathbf{a}, E_{b}$ by $\mathbf{b}$ etc. For example, Eq. (2.2) can now be written

$$
\mathbf{b}=\mathbf{T a},
$$

with $\mathbf{T}$ the transmission matrix. The incident field is normalized so that $\|\mathbf{a}\|=1$.

\section{Wirtinger calculus}

To analyze convergence to a local maximum, we will expand the system around a fixed point $\mathbf{a}_{*}$. Here a technical complication arises: due to the complex conjugate in $S$, it is not a holomorphic function of a, so the complex derivative $\partial S / \partial \mathbf{a}$ does not exist.

In order to avoid this complication, we use Wirtinger calculus to calculate the derivatives [20]. In Wirtinger calculus, $S(\mathbf{a})$ is replaced by a function $S(\mathbf{a}, \overline{\mathbf{a}})$, where $\mathbf{a}$ and $\overline{\mathbf{a}}$ are independent variables: technically, $\overline{\mathbf{a}}$ is not equal to the complex conjugate of $\mathbf{a}$. However, as long as we restrict ourselves to only evaluate $S(\mathbf{a}, \overline{\mathbf{a}})$ in the subspace $\overline{\mathbf{a}}=\mathbf{a}^{*}$, we can perform all derivatives in the usual manner and obtain correct results. Using the compact notation and Wirtinger calculus, we can now write Eq. (2.1) as

$$
S(\mathbf{a}, \overline{\mathbf{a}})=\sum_{b}^{M}\left[\left(\mathbf{T}^{*} \overline{\mathbf{a}}\right)_{b}(\mathbf{T a})_{b}\right]^{n},
$$

where ()$_{b}$ denotes the $b$-th element of the vector in the parentheses. As an example, we use Wirtinger calculus to calculate the first order Taylor expansion of $S$ for small perturbations $\Delta$ around a

$$
S(\mathbf{a}+\Delta, \overline{\mathbf{a}}+\bar{\Delta})=S(\mathbf{a}, \overline{\mathbf{a}})+\frac{\partial S}{\partial \mathbf{a}} \Delta+\frac{\partial S}{\partial \overline{\mathbf{a}}} \bar{\Delta}+O\left(\|\Delta\|^{2}\right) .
$$

We can now evaluate the derivative towards component $a_{i}$ of the incident field $\mathbf{a}$

$$
\frac{\partial S}{\partial a_{i}}=n \sum_{b}^{M}\left[\left(\mathbf{T}^{*} \overline{\mathbf{a}}\right)_{b}(\mathbf{T a})_{b}\right]^{n-1} t_{b i}=\sum_{b}^{M} w_{b}^{*} t_{b i},
$$

where the elements of $\mathbf{w}$ and $\overline{\mathbf{w}}$ are given by

$$
w_{b}(\mathbf{a}, \overline{\mathbf{a}})=n(\mathbf{T a})_{b}^{n}\left(\mathbf{T}^{*} \overline{\mathbf{a}}\right)_{b}^{(n-1)} \quad \text { and } \quad \bar{w}_{b}(\mathbf{a}, \overline{\mathbf{a}})=n\left(\mathbf{T}^{*} \overline{\mathbf{a}}\right)_{b}^{n}(\mathbf{T a})_{b}^{(n-1)} .
$$

By evaluating the derivative for all elements $a_{i}$, we get the vector derivatives

$$
\frac{\partial S}{\partial \mathbf{a}}=\overline{\mathbf{w}}^{T} \mathbf{T} \quad \text { and } \quad \frac{\partial S}{\partial \overline{\mathbf{a}}}=\mathbf{w}^{T} \mathbf{T}^{*}
$$

We can use the Taylor expansion Eq. (2.15) to calculate what happens during the optimization process, when the phase of a single element of the incident field is changed. The perturbation corresponding to changing the phase of segment $a^{\prime}$ is given by

$$
\Delta_{i}=\left\{\begin{array}{ll}
a_{i}\left(e^{i \phi}-1\right) & \text { for } i=a^{\prime} \\
0 & \text { otherwise }
\end{array} .\right.
$$

Inserting $\Delta$ into Eq. (2.15) gives Eq. (2.4) from the main text. 


\section{Attractivity of fixed point}

The algorithm in Eq. (2.5) can be thought of as a cyclical series of mappings $\mathbf{a}_{(k)} \rightarrow \mathbf{b}_{(k)} \rightarrow$ $\mathbf{w}_{(k)} \rightarrow \mathbf{T}^{\dagger} \mathbf{w}_{(k)} \rightarrow \mathbf{a}_{(k+1)}$, etc., where $\mathbf{T}^{\dagger}$ is the conjugate transpose of $\mathbf{T}$. We rewrite Eq. (2.5) as

$$
\mathbf{f}(\mathbf{a}, \overline{\mathbf{a}})=\frac{\mathbf{T}^{\dagger} \mathbf{w}(\mathbf{a}, \overline{\mathbf{a}})}{\left\|\mathbf{T}^{\dagger} \mathbf{w}(\mathbf{a}, \overline{\mathbf{a}})\right\|}
$$

where $\mathbf{f}$ now defines the mapping from $\mathbf{a}_{(k)}$ to $\mathbf{a}_{(k+1)}$. Using Wirtinger calculus, we can make a first order approximation of the mapping for a small perturbation $\Delta$ around a fixed point of the mapping, $\mathbf{a}_{*}=\mathbf{f}\left(\mathbf{a}_{*}\right)$.

$$
\mathbf{f}\left(\mathbf{a}_{*}+\Delta, \overline{\mathbf{a}}_{*}+\bar{\Delta}\right) \approx \mathbf{f}\left(\mathbf{a}_{*}, \overline{\mathbf{a}}_{*}\right)+\left[\begin{array}{ll}
\frac{\partial \mathbf{f}}{\partial \mathbf{a}} & \frac{\partial \mathbf{f}}{\partial \overline{\mathbf{a}}} \\
\frac{\partial \overline{\mathbf{f}}}{\partial \mathbf{a}} & \frac{\partial \overline{\mathbf{f}}}{\partial \overline{\mathbf{a}}}
\end{array}\right]_{\frac{\mathbf{a}=\mathbf{a}_{*}}{\overline{\mathbf{a}}=\mathbf{a}_{*}}}\left[\begin{array}{l}
\Delta \\
\Delta
\end{array}\right],
$$

where the matrix is $J_{f}\left(\mathbf{a}_{*}\right)$ : the Jacobian of $\mathbf{f}$, evaluated at the fixed point $\mathbf{a}_{*}$. When the fixed point is attractive in some finite region, each iteration brings $\mathbf{a}$ closer to $\mathbf{a}_{*}$, so we must have $\left\|\mathbf{a}_{(k+1)}-\mathbf{a}_{*}\right\| \leq q\left\|\mathbf{a}_{(k)}-\mathbf{a}_{*}\right\|$, with $0 \leq q<1$. From Eq. (2.21), we see that this condition is equivalent to saying that the spectral radius of the Jacobian $\rho\left(J_{f}\right)<1$. We will show below that this condition is always met at local maxima of $S$.

From the definition of $\mathbf{f}$ it is clear that any perturbation in the direction of $\mathbf{a}_{*}$ will have no effect at all. Therefore, we restrict ourselves to perturbations perpendicular to $\mathbf{a}_{*}$, i.e. $\bar{\Delta}^{T} \mathbf{a}_{*}=0$, hence $\bar{\Delta}^{T} \mathbf{T}^{\dagger} \mathbf{w}_{*}=0$. Under this condition, we can find a simple expression for terms of the form $\bar{\Delta}^{T} \partial \mathbf{f} / \partial \mathbf{a}$

$$
\left.\bar{\Delta}^{T} \frac{\partial \mathbf{f}}{\partial \mathbf{a}}\right|_{\substack{\mathbf{a}=\mathbf{a}_{*} \\ \overline{\mathbf{a}}=\overline{\mathbf{a}}_{*}}}=\bar{\Delta}^{T}\left[\frac{\mathbf{T}^{\dagger}}{\left\|\mathbf{T}^{\dagger} \mathbf{w}\right\|} \frac{\partial \mathbf{w}}{\partial \mathbf{a}}+\mathbf{T}^{\dagger} \mathbf{w} \frac{\partial}{\partial \mathbf{a}} \frac{1}{\left\|\mathbf{T}^{\dagger} \mathbf{w}\right\|}\right]_{\substack{\mathbf{a}=\mathbf{a}_{*} \\ \mathbf{a}=\mathbf{a}_{*}}}=\left.\bar{\Delta}^{T} \frac{\mathbf{T}^{\dagger}}{\left\|\mathbf{T}^{\dagger} \mathbf{w}\right\|} \frac{\partial \mathbf{w}}{\partial \mathbf{a}}\right|_{\substack{\mathbf{a}=\mathbf{a}_{*} \\ \overline{\mathbf{a}}=\overline{\mathbf{a}}_{*}}},
$$

which we will use in the next section. Here the product rule was used in the first step, and orthogonality of $\Delta$ and $\mathbf{a}_{*}$ was used in the second step.

\section{Local maximum}

To find local maxima of $S$ under the constraint that $\|\mathbf{a}\|=1$, we apply the method of Lagrange multipliers and minimize the Lagrange function

$$
S_{L}(\mathbf{a}, \overline{\mathbf{a}})=S(\mathbf{a}, \overline{\mathbf{a}})-\lambda\left(\overline{\mathbf{a}}^{T} \mathbf{a}-1\right),
$$

where $\lambda$ is a Lagrange multiplier and $\overline{\mathbf{a}}^{T} \mathbf{a}-1$ represents the constraint that $\|\mathbf{a}\|=1$.

The first order conditions for a local maximum follow by equating the first derivatives of $S_{L}$ to zero, giving

$$
\begin{aligned}
\overline{\mathbf{w}}_{*}^{T} \mathbf{T}-\lambda \overline{\mathbf{a}}_{*}^{T} & =0 \\
\mathbf{w}_{*}^{T} \mathbf{T}^{*}-\lambda \mathbf{a}_{*}^{T} & =0 \\
\overline{\mathbf{a}}_{*}^{T} \mathbf{a}_{*}-1 & =0,
\end{aligned}
$$


with the solution $\lambda=\left\|\mathbf{T}^{\dagger} \mathbf{w}_{*}\right\|$ and $\mathbf{a}_{*}=\mathbf{T}^{\dagger} \mathbf{w}_{*} / \lambda$, proving that a fixed point of $\mathbf{f}$ is also a critical point of $S_{L}$. In order to prove that this stationary point is a local maximum (and not a local minimum or a saddle point), we need show that any small perturbation that maintains the first order conditions decreases the value of $S_{L}$. In order to do so, we evaluate the second derivative (the Hessian matrix $\mathbf{H}$ ) of $S_{L}$ considering only perturbations perpendicular to $\mathbf{a}_{*}$, i.e. perturbations that maintain the constraint $\|\mathbf{a}\|=1$ to the first order. Using Eq. (2.18), we arrive at

$$
\mathbf{H} \equiv\left[\begin{array}{cc}
\frac{\partial^{2} S_{L}}{\partial \mathbf{a} \partial \overline{\mathbf{a}}} & \frac{\partial^{2} S_{L}}{\partial \overline{\mathbf{a}}^{2}} \\
\frac{\partial^{2} S_{L}}{\partial \mathbf{a}^{2}} & \frac{\partial^{2} S_{L}}{\partial \overline{\mathbf{a}} \partial \mathbf{a}}
\end{array}\right]_{\overline{\mathbf{a}}=\mathbf{a}_{*}}=\left[\begin{array}{cc}
\mathbf{T}^{\dagger} \frac{\partial \mathbf{w}}{\partial \mathbf{a}}-\lambda & \mathbf{T}^{\dagger} \frac{\partial \mathbf{w}}{\partial \overline{\mathbf{a}}} \\
\mathbf{T}^{T} \frac{\partial \overline{\mathbf{w}}}{\partial \mathbf{a}} & \mathbf{T}^{T} \frac{\partial \overline{\mathbf{w}}}{\partial \overline{\mathbf{a}}}-\lambda
\end{array}\right]_{\overline{\mathbf{a}=\mathbf{a}_{*}}} .
$$

Using Eq. (2.22), we find

$$
\left[\begin{array}{l}
\Delta \\
\Delta
\end{array}\right]^{T} \mathbf{H}\left[\frac{\Delta}{\Delta}\right]=\left[\begin{array}{l}
\Delta \\
\Delta
\end{array}\right]^{T} \lambda\left(J_{f}-\mathbf{I}\right)\left[\frac{\Delta}{\Delta}\right]<0
$$

with $\mathbf{I}$ the identity matrix. Since $\rho\left(J_{f}\right)<1$, from Eq. (2.28), $J_{f}-\mathbf{I}$ is negative definite, proving the last step: every perturbation $\Delta$ decreases the signal. Hence, every fixed point $\mathbf{a}_{*}$ corresponds to a local maximum of the constrained optimization problem.

\section{Conclusion}

This final result in Eq. (2.28) shows that any small perturbation around the fixed point decreases the signal. In conclusion, we demonstrated that, when $\mathbf{a}_{*}$ is an attractive fixed point of mapping $\mathbf{f}$, the resulting signal $S\left(\mathbf{a}_{*}, \overline{\mathbf{a}}_{*}\right)$ must be a local maximum. The converse is also true, since both statements are equivalent to the condition that $\rho\left(J_{f}\right)<1$. Finally, as detailed in the main text, even though the algorithm maximizes $S$, such a maximum does not necessarily correspond to a focus.

\subsubsection{Derivation of the blind focusing statistical model}

In this appendix, we derive Eq. (2.7), the complex normal distribution of the optimized target field when blind focusing through a strongly scattering sample. We start by constructing the optimized incident field, which is given in Eq. (2.5). In an imperfect wavefront shaping setup, the quality of the wavefront modulation is described by the fidelity, $|\gamma|^{2}$. The constructed input field is given by $\hat{E}_{a}^{(k+1)}=\gamma E_{a}^{(k+1)}+\sqrt{1-|\gamma|^{2}} \zeta_{a}$, where $E_{a}^{(k+1)}$ is the desired input field and $\zeta_{a}$ is a normalized field, which is by definition orthogonal to $E_{a}^{(k+1)}$ [21]. When this imperfect input field is inserted into Eq. (2.2), we find that the constructed optimized target field becomes

$$
\hat{E}_{b}^{(k+1)}=\gamma c^{(k+1)} \sum_{a}^{N} \sum_{b^{\prime}}^{M} t_{b a} t_{b^{\prime} a}^{*} W_{b^{\prime}}^{(k)}+\sqrt{1-|\gamma|^{2}} \zeta_{b} .
$$

Here, $\zeta_{b} \equiv \sum_{a}^{N} t_{b a} \zeta_{a}$ which is assumed to be an uncorrelated scattered field with $\left\langle\zeta_{b}\right\rangle=0$ and $\operatorname{var}\left(\zeta_{b}\right)=\left\langle\left|t_{b a}\right|^{2}\right\rangle$. Note that for a perfect setup with $\gamma=1, \hat{E}_{b}^{(k+1)}=E_{b}^{(k+1)}$ (Eq. (2.6)). We recognize that Eq. (2.29) can be written as a sum over $N$ independent random variables $\chi_{a}$

$$
\hat{E}_{b}^{(k+1)}=\gamma c^{(k+1)} \sum_{a}^{N} \chi_{a}^{(k)}+\sqrt{1-|\gamma|^{2}} \zeta_{b} \quad \text { with } \quad \chi_{a}^{(k)} \equiv \sum_{b^{\prime}}^{M} t_{b a} t_{b^{\prime} a}^{*} W_{b^{\prime}}^{(k)} .
$$


When $N$ is large, by the central limit theorem $\hat{E}_{b}^{(k+1)}$ has a complex normal distribution.

To find the average and variance of $\hat{E}_{b}^{(k+1)}$, we start by calculating the first and second raw moments of the terms $\chi_{a}$. As stated in the main text, we assume that $W_{b}^{(k)}$ is known and independent of $\mathbf{T}$, and that $\left\langle t_{b a} t_{b^{\prime} a^{\prime}}^{*}\right\rangle=\delta_{b^{\prime} b} \delta_{a^{\prime} a}\left\langle\left|t_{b a}\right|^{2}\right\rangle$. Under these assumptions, the first moment is given by

$$
\left\langle\chi_{a}^{(k)}\right\rangle=\sum_{b^{\prime}}^{M} W_{b^{\prime}}^{(k)}\left\langle t_{b a} t_{b^{\prime} a}^{*}\right\rangle=W_{b}^{(k)}\left\langle\left|t_{b a}\right|^{2}\right\rangle
$$

and the second moment

$$
\begin{aligned}
\left\langle\left|\chi_{a}^{(k)}\right|^{2}\right\rangle & =\sum_{b^{\prime}}^{M} \sum_{b^{\prime \prime}}^{M} W_{b^{\prime}}^{(k)} W_{b^{\prime \prime}}^{(k) *}\left\langle\left|t_{b a}\right|^{2} t_{b^{\prime} a}^{*} t_{b^{\prime \prime} a}\right\rangle \\
& =\sum_{b^{\prime}}^{M}\left|W_{b^{\prime}}^{(k)}\right|^{2}\left\langle\left|t_{b a}\right|^{2}\left|t_{b^{\prime} a}\right|^{2}\right\rangle \\
& =\sum_{b^{\prime} \neq b}^{M}\left|W_{b^{\prime}}^{(k)}\right|^{2}\left\langle\left|t_{b a}\right|^{2}\right\rangle\left\langle\left|t_{b^{\prime} a}\right|^{2}\right\rangle+\left|W_{b}^{(k)}\right|^{2}\left\langle\left|t_{b a}\right|^{4}\right\rangle .
\end{aligned}
$$

Realizing that for a Gaussian distribution, $\left\langle\left|t_{b a}\right|^{4}\right\rangle=2\left\langle\left|t_{b a}\right|^{2}\right\rangle^{2}$, we find

$$
\operatorname{var}\left(\chi_{a}^{(k)}\right)=\left\langle\left|\chi_{a}^{(k)}\right|^{2}\right\rangle-\left|\left\langle\chi_{a}^{(k)}\right\rangle\right|^{2}=\left\langle\left|t_{b a}\right|^{2}\right\rangle^{2} \sum_{b^{\prime}}^{M}\left|W_{b^{\prime}}^{(k)}\right|^{2}
$$

Next, we calculate the value of $c^{(k+1)}$, which was introduced in Eq. (2.5) to normalize the total incident intensity after the optimization. We assume that, for large $N$, the normalization factor is self-averaging, such that

$$
c^{(k+1)}=\sqrt{\frac{1}{\sum_{a}^{N}\left|\sum_{b}^{M} t_{b a}^{*} W_{b}^{(k)}\right|^{2}}} \approx \sqrt{\frac{1}{N\left\langle\left|t_{b a}\right|^{2}\right\rangle \sum_{b}^{M}\left|W_{b}^{(k)}\right|^{2}}} .
$$

To obtain the mean of $\hat{E}_{b}^{(k+1)}$, we can simply add the means of $\chi_{a}$ and $\zeta_{b}$, since the two variables are uncorrelated. We calculate the optimized field average by inserting Eq. (2.31) and $\left\langle\zeta_{b}^{(k)}\right\rangle=0$ into Eq. (2.30)

$$
\left\langle\hat{E}_{b}^{(k+1)}\right\rangle=\gamma c^{(k+1)} \sum_{a}^{N}\left\langle\chi_{a}^{(k)}\right\rangle+\sqrt{1-|\gamma|^{2}}\left\langle\zeta_{b}\right\rangle=\frac{W_{b}^{(k)}}{\sqrt{\sum_{b^{\prime}}^{M}\left|W_{b^{\prime}}^{(k)}\right|^{2}}} \gamma \sqrt{N\left\langle\left|t_{b a}\right|^{2}\right\rangle}
$$

Similarly, we can calculate the variance of $\hat{E}_{b}^{(k+1)}$ by adding $\operatorname{var}\left(\chi_{a}^{(k)}\right)$ (from Eq. (2.35)) and $\operatorname{var}\left(\zeta_{b}\right)=\left\langle\left|t_{b a}\right|^{2}\right\rangle$ in the following manner

$$
\begin{aligned}
\operatorname{var}\left(\hat{E}_{b}^{(k+1)}\right) & =|\gamma|^{2}\left(c^{(k+1)}\right)^{2} \sum_{a}^{N} \operatorname{var}\left(\chi_{a}^{(k)}\right)+\left(1-|\gamma|^{2}\right) \operatorname{var}\left(\zeta_{b}\right) \\
& =|\gamma|^{2} \frac{N\left\langle\left|t_{b a}\right|^{2}\right\rangle^{2} \sum_{b^{\prime}}^{M}\left|W_{b^{\prime}}^{(k)}\right|^{2}}{N\left\langle\left|t_{b a}\right|^{2}\right\rangle \sum_{b^{\prime \prime}}^{M}\left|W_{b^{\prime \prime}}^{(k)}\right|^{2}}+\left(1-|\gamma|^{2}\right)\left\langle\left|t_{b a}\right|^{2}\right\rangle \\
& =\left\langle\left|t_{b a}\right|^{2}\right\rangle
\end{aligned}
$$


When we substitute $\mu_{b}^{(k+1)} \equiv\left\langle\hat{E}_{b}^{(k+1)}\right\rangle$ and $I_{0} \equiv \operatorname{var}\left(\hat{E}_{b}^{(k+1)}\right)$, we arrive at the expressions found in Eq. (2.8).

\subsubsection{Derivation of the blind focusing requirements}

In this section, we calculate the minimal requirements for the formation of a focus using the blind focus method. We assume that, when a focus is formed, only the intensity at the focus location is enhanced and that all other targets have an exponentially distributed enhancement with an average of 1 . A focus can be formed when the expected focus enhancement of the next iteration $\left\langle\eta^{(1)}\right\rangle$ (as described by Eq. (2.11)) is larger than or equal to the current focus enhancement, for some $\eta^{(0)}>1$. For simplification, we instead consider $\left\langle\eta^{(1)}\right\rangle \geq \eta^{(0)}+1$, which is a slightly more restrictive condition. We can write this condition as

$$
\begin{aligned}
N|\gamma|^{2} \frac{\left(\eta^{(0)}\right)^{2 n-1}}{\left(\eta^{(0)}\right)^{2 n-1}+(2 n-1) !(M-1)}+1 & \geq \eta^{(0)}+1 \\
-\left(\eta^{(0)}\right)^{2 n-1}+N|\gamma|^{2}\left(\eta^{(0)}\right)^{2 n-2} & \geq(2 n-1) !(M-1) .
\end{aligned}
$$

Next, we derive the minimum requirements for this condition to be satisfied. Therefore, we proceed by finding

$$
\max _{\eta^{(0)}}\left(-\left(\eta^{(0)}\right)^{2 n-1}+N|\gamma|^{2}\left(\eta^{(0)}\right)^{2 n-2}\right)
$$

to find out in which cases this maximum value satisfies the condition in Eq. (2.42). We maximize this function by equating the derivative of this function towards $\eta^{(0)}$ to zero, giving

$$
\begin{aligned}
(2 n-1)\left(\eta^{(0)}\right)^{2 n-2} & =(2 n-2) N|\gamma|^{2}\left(\eta^{(0)}\right)^{2 n-3} \\
\eta^{(0)} & =\frac{2 n-2}{2 n-1} N|\gamma|^{2} .
\end{aligned}
$$

We insert this maximized value for $\eta^{(0)}$ into Eq. (2.42)

$$
-\left(\frac{2 n-2}{2 n-1} N|\gamma|^{2}\right)^{2 n-1}+N|\gamma|^{2}\left(\frac{2 n-2}{2 n-1} N|\gamma|^{2}\right)^{2 n-2} \geq(2 n-1) !(M-1) .
$$

Given $N,|\gamma|^{2}$ and $n$, we solve Eq. (2.46) for $M$

$$
\begin{aligned}
(2 n-1) !(M-1) & <\left(\frac{2 n-2}{2 n-1}\right)^{2 n-2}\left(1-\frac{2 n-2}{2 n-1}\right)\left(N|\gamma|^{2}\right)^{2 n-1} \\
(2 n-1) !(M-1) & <\frac{(2 n-2)^{2 n-2}}{(2 n-1)^{2 n-1}}\left(N|\gamma|^{2}\right)^{2 n-1} \\
M & <\frac{(2 n-2)^{2 n-2}}{(2 n-1) !(2 n-1)^{2 n-1}}\left(|\gamma|^{2} N\right)^{2 n-1}+1
\end{aligned}
$$

This final expression is the upper-limit for $M$, which corresponds to the parameter $M_{\max }$ as given in Eq. (2.12). Whenever $M<M_{\max }$, the condition in Eq. (2.42) will be satisfied, which means that, on average, blind focusing is indeed able to form a a single focus. 


\section{Acknowledgments}

This work was supported by the European Research Council (ERC-2016-StG-678919). The authors would like to thank Yoeri Boink for the helpful discussions and Tom Knop for his aid in the fabrication of the samples.

\section{Bibliography}

[1] I. M. Vellekoop, "Feedback-based wavefront shaping”, Opt. Express 23, 12189-12206 (2015).

[2] R. Horstmeyer, H. Ruan, and C. Yang, "Guidestar-assisted wavefront-shaping methods for focusing light into biological tissue”, Nat. Photonics 9, 563-571 (2015).

[3] K. Dholakia and T. Čižmár, "Shaping the future of manipulation", Nat. Photonics 5, 335-342 (2011).

[4] H. Ruan, J. Brake, J. E. Robinson, Y. Liu, M. Jang, C. Xiao, C. Zhou, V. Gradinaru, and C. Yang, "Deep tissue optical focusing and optogenetic modulation with time-reversed ultrasonically encoded light", Sci. Adv. 3, eaao5520 (2017).

[5] J.-H. Park, W. Sun, and M. Cui, "High-resolution in vivo imaging of mouse brain through the intact skull”, Proc. Natl. Acad. Sci. U. S. A. 112, 9236-9241 (2015).

[6] Y. Shen, Y. Liu, C. Ma, and L. V. Wang, "Focusing light through biological tissue and tissue-mimicking phantoms up to $9.6 \mathrm{~cm}$ in thickness with digital optical phase conjugation", J. Biomed. Opt. 21, 085001 (2016).

[7] J. Tang, R. N. Germain, and M. Cui, "Superpenetration optical microscopy by iterative multiphoton adaptive compensation technique", Proc. Natl. Acad. Sci. U. S. A. 109, 8434-8439 (2012).

[8] D. Sinefeld, H. P. Paudel, D. G. Ouzounov, T. G. Bifano, and C. Xu, “Adaptive optics in multiphoton microscopy: comparison of two, three and four photon fluorescence", Opt. Express 23, 31472-31483 (2015).

[9] D. E. Milkie, E. Betzig, and N. Ji, "Pupil-segmentation-based adaptive optical microscopy with full-pupil illumination”, Opt. Lett. 36, 4206-4208 (2011).

[10] O. Katz, E. Small, Y. Guan, and Y. Silberberg, "Noninvasive nonlinear focusing and imaging through strongly scattering turbid layers", Optica 1, 170-174 (2014).

[11] O. Katz, E. Small, Y. Bromberg, and Y. Silberberg, "Focusing and compression of ultrashort pulses through scattering media", Nat. Photonics 5, 372-377 (2011).

[12] J. Aulbach, B. Gjonaj, P. Johnson, and A. Lagendijk, "Spatiotemporal focusing in opaque scattering media by wave front shaping with nonlinear feedback", Opt. Express 20, 29237-29251 (2012). 
[13] D. B. Conkey, A. N. Brown, A. M. Caravaca-Aguirre, and R. Piestun, "Genetic algorithm optimization for focusing through turbid media in noisy environments", Opt. Express 20, 4840-4849 (2012).

[14] I. M. Vellekoop and A. P. Mosk, "Focusing coherent light through opaque strongly scattering media", Opt. Lett. 32, 2309-2311 (2007).

[15] J. Bosch, S. A. Goorden, and A. P. Mosk, "Frequency width of open channels in multiple scattering media", Opt. Express 24, 26472-26478 (2016).

[16] I. N. Papadopoulos, J.-S. Jouhanneau, J. F. A. Poulet, and B. Judkewitz, "Scattering compensation by focus scanning holographic aberration probing (F-SHARP)", Nat. Photonics 11, 116-123 (2017).

[17] J. W. Goodman, Speckle phenomena in optics, chapter 3 (Roberts \& Company) (2007).

[18] E. G. van Putten, "Disorder-enhanced imaging with spatially controlled light", Ph.D. thesis, University of Twente (2011).

[19] S. Rotter and S. Gigan, "Light fields in complex media: mesoscopic scattering meets wave control”, Rev. Mod. Phys. 89, 015005 (2017).

[20] R. F. H. Fisher, Precoding and signal shaping for digital transmission, chapter Appendix A (John Wiley \& Sons, Inc.) (2002).

[21] I. M. Vellekoop and A. P. Mosk, "Universal optimal transmission of light through disordered materials”, Phys. Rev. Lett. 101, 120601 (2008). 


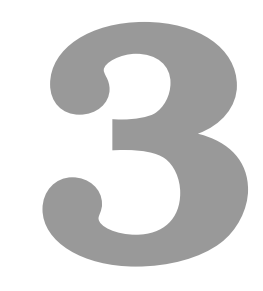

\section{The generalized optical memory effect}

The optical memory effect is a well-known type of tilt/tilt wave correlation that is observed in coherent fields, allowing control over scattered light through thin and diffusive materials. Here we show that the optical memory effect is a special case of a more general class of combined shift/tilt correlations, occurring in media of arbitrary geometry. We experimentally demonstrate the existence of these correlations, and provide an analytical framework that allows us to predict and understand this class of scattering correlations. This 'generalized optical memory effect' can be utilized for maximizing the imaging field-of-view of deep tissue imaging techniques such as phase conjugation and adaptive optics.

\subsection{Introduction}

It is challenging to record clear images from deep within biological tissue. As an optical field passes through tissue its spatial profile becomes randomly perturbed, resulting in a blurry image of the features that lie underneath. Luckily, even highly scattered optical fields still maintain a certain degree of correlation. Such scattering-based correlations have recently enabled new 'hidden imaging' approaches [1-4], which reconstruct clear images from behind diffusive materials. These prior investigations have primarily exploited what is traditionally referred to as the optical memory effect $[5,6]$. This effect predicts that a scattered wavefront will tilt, but otherwise not change, when the beam incident upon a scattering material is also tilted by the same amount (see Fig. 3.1a). These correlations have been observed through both thin isotropically scattering screens [7] as well as thick forward scattering tissue [8].

Recently, we reported a new type of 'shift' memory effect, illustrated in Fig. 3.1b, that occurs primarily in anisotropically scattering media [9]. This form of correlation is especially

This chapter has been published as: [G. Osnabrugge, R. Horstmeyer, I.N. Papadopoulos, B. Judkewitz and I.M. Vellekoop, Optica 8, 886-892 (2017)]. 


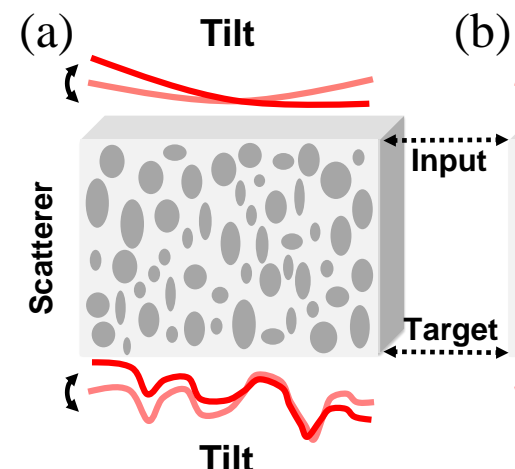

(b)
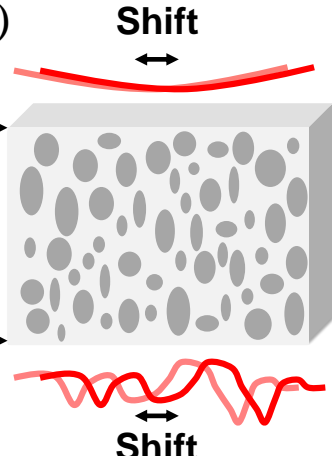

(c)

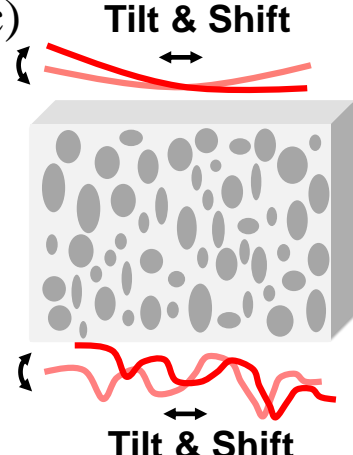

Figure 3.1: Three different types of spatial correlations in disordered media. (a) The optical 'tilt' memory effect [6], where an input wavefront tilt leads to a tilt at the target plane. (b) The anisotropic 'shift' memory effect [9], where an input wavefront shift also shifts the target plane wavefront. (c) Our new generalized memory effect, relying on both tilts and shifts, which can maximize correlations along the target plane for the optimal scanning scheme.

important in biomedical imaging, as it offers the ability to physically shift (as opposed to tilt) a focal spot formed deep within scattering tissue by translating an incident optical beam. However, neither of the two reported types of correlation could fully explain the observations seen in thick biological tissue [8].

Here, we show how the optical 'tilt' and 'shift' memory effects are non-trivially intertwined. In fact, the two effects are manifestations of one and the same general source of correlation within a scattering process which depends upon how an incident wavefront is both tilted and shifted (see Fig. 3.1c). Our new 'generalized memory effect' model offers a complete description of these combined shift/tilt correlations present within scattering media. With our model we are able to explain the unexpectedly large optical memory effect observed within biological tissue, estimate the size of the isoplanatic patch in microscopy, and optimize the design of adaptive optics microscopes [10]. Additionally, we develop and verify a Fokker-Planck light propagation model that predicts an optimal imaging/scanning strategy for a given forward scattering sample based on its scattering response. While our model applies to coherent waves in general, we limit our attention to the optical regime.

We start out by presenting our model for the generalized memory effect. We describe how to predict the amount of expected correlation through a given slab of scattering material as function of both position and wavevector. Then, we discuss the direct applications of our findings to adaptive optics before presenting experimental memory effect measurements.

\subsection{Model}

In our model, we consider propagation of monochromatic and coherent light from an "input" plane $a$ to a "target" plane $b$. We limit ourselves to scalar waves. The forward-propagating field at the "input" surface, $E_{a}$, and at the "target" plane, $E_{b}$, are coupled through

$$
E_{b}\left(\mathbf{r}_{b}\right)=\int T\left(\mathbf{r}_{b}, \mathbf{r}_{a}\right) E_{a}\left(\mathbf{r}_{a}\right) d^{2} \mathbf{r}_{a} .
$$


It should be noted here that our model is completely general and does not make any assumptions about the location or orientation of the two planes, nor about the symmetry, shape, or other properties of the scattering sample. For example, for transmission through a slab of scattering material, the target plane is located at the back surface of the sample and $T\left(\mathbf{r}_{b}, \mathbf{r}_{a}\right)$ is the sample's transmission matrix. Conversely, in a biomedical microscope setup, the target plane is typically located inside a scattering sample like tissue, and $T$ is a field propagator connecting position $\mathbf{r}_{a}$ to a position $\mathbf{r}_{b}$ located inside the tissue.

At this point let us define exactly what type of correlations we are interested in. Suppose that an input field $E_{a}$ results in a target field $E_{b}$. We hypothesize that when we shift/tilt the incident field with respect to the medium, the new target field will also experience a shift/tilt and also remain similar to the original target field. This similarity can be expressed as a correlation function involving the original matrix $T$ and a new matrix $\tilde{T}$ associated with the shifted/tilted field. We describe 'tilting' the incident wave as a multiplication with a phase ramp, $\exp \left(i \Delta \mathbf{k}_{a} \cdot \mathbf{r}_{a}\right)$, resulting in a wavefront that is tilted by $\Delta \mathbf{k}_{a}$ with respect to plane $a$. Likewise, we describe the corresponding tilt at the target plane via a multiplication with $\exp \left(-i \Delta \mathbf{k}_{b} \cdot \mathbf{r}_{b}\right)$.

We may now describe the new shifted and tilted situation by writing the corresponding shift/tilt matrix as $\tilde{T}\left(\mathbf{r}_{b}, \mathbf{r}_{a}\right)=\exp \left(-i \Delta \mathbf{k}_{b} \cdot \mathbf{r}_{b}\right) T\left(\mathbf{r}_{b}+\Delta \mathbf{r}_{b}, \mathbf{r}_{a}+\Delta \mathbf{r}_{a}\right) \exp \left(i \Delta \mathbf{k}_{a} \cdot \mathbf{r}_{a}\right)$, where $\tilde{T}$ is shifted by $\Delta \mathbf{r}_{a}$ and $\Delta \mathbf{r}_{b}$ at the input and target plane, respectively. By calculating the ensemble averaged value $\left\langle T \tilde{T}^{*}\right\rangle$, we can find the corresponding shift/tilt correlation coefficient. However, we found that the problem is expressed more naturally if we first introduce the center-difference coordinates $\mathbf{r}_{a}^{+} \equiv \mathbf{r}_{a}+\Delta \mathbf{r}_{a} / 2, \mathbf{r}_{a}^{-} \equiv \mathbf{r}_{a}-\Delta \mathbf{r}_{a} / 2$ (and the same for $\mathbf{r}_{b}$ ). Using the same reasoning as above, we now find a symmetric expression for our generalized shift-tilt correlation function:

$$
C\left(\Delta \mathbf{r}_{b}, \Delta \mathbf{k}_{b} ; \Delta \mathbf{r}_{a}, \Delta \mathbf{k}_{a}\right) \equiv \iint\left\langle T\left(\mathbf{r}_{b}^{+}, \mathbf{r}_{a}^{+}\right) T^{*}\left(\mathbf{r}_{b}^{-}, \mathbf{r}_{a}^{-}\right)\right\rangle e^{i\left(\Delta \mathbf{k}_{a} \cdot \mathbf{r}_{a}-\Delta \mathbf{k}_{b} \cdot \mathbf{r}_{b}\right)} d^{2} \mathbf{r}_{a} d^{2} \mathbf{r}_{b}
$$

In the special case that $\Delta \mathbf{k}_{a}=\Delta \mathbf{k}_{b}$ and $\Delta \mathbf{r}_{a}=\Delta \mathbf{r}_{b}=0$, Eq. (3.2) reduces to the optical 'tilt' memory effect [6], whereas for $\Delta \mathbf{r}_{a}=\Delta \mathbf{r}_{b}$ and $\Delta \mathbf{k}_{a}=\Delta \mathbf{k}_{b}=0$, the correlation function corresponds to the anisotropic 'shift' memory effect described in Ref. [9]. Later we will show that the generalized correlation function is not simply a trivial combination of the separate shift and tilt memory effects.

Before proceeding to calculate $C$ in terms of the sample properties, we introduce the Wigner distribution function (WDF), which describes the optical field as distribution in a joint phase space of two Fourier-conjugate variables $[11,12]$. In our case, the variables of interest are space (r) and wavevector $(\mathbf{k})$ :

$$
W(\mathbf{r}, \mathbf{k}) \equiv \int E\left(\mathbf{r}^{+}\right) E^{*}\left(\mathbf{r}^{-}\right) e^{-i \mathbf{k} \cdot \Delta \mathbf{r}} d^{2} \Delta \mathbf{r} .
$$

We choose to work with the WDF because it will allow us to convert Eq. (3.1) into a function of both space and wavevector, which we may easily connect to our new correlation function $C$ of similar variables. In the paraxial approximation, the Wigner distribution function is effectively equivalent to the light field [13], which describes the amount of optical power at point $\mathbf{r}$ that is propagating in direction $\mathbf{k}$, like a spatio-angular plot of light rays at various locations propagating in different directions.

To describe the scattering of incident light over space and wavevector, we introduce the 'light field transmission function', $P$. This function maps the incident light field $W_{a}\left(\mathbf{r}_{a}, \mathbf{k}_{a}\right)$ 
to the transmitted light field $W_{b}\left(\mathbf{r}_{b}, \mathbf{k}_{b}\right)$ at the target plane:

$$
W_{b}\left(\mathbf{r}_{b}, \mathbf{k}_{b}\right)=\frac{1}{(2 \pi)^{2}} \iint P\left(\mathbf{r}_{b}, \mathbf{k}_{b} ; \mathbf{r}_{a}, \mathbf{k}_{a}\right) W_{a}\left(\mathbf{r}_{a}, \mathbf{k}_{a}\right) d^{2} \mathbf{r}_{a} d^{2} \mathbf{k}_{a}
$$

Eq. (3.4) is the phase space equivalent of Eq. (3.1). In Appendix 3.7.1, we show that $P$ takes the form of a double-Wigner distribution of the transmission matrix:

$$
P\left(\mathbf{r}_{b}, \mathbf{k}_{b} ; \mathbf{r}_{a}, \mathbf{k}_{a}\right) \equiv \iint T\left(\mathbf{r}_{b}^{+}, \mathbf{r}_{a}^{+}\right) T^{*}\left(\mathbf{r}_{b}^{-}, \mathbf{r}_{a}^{-}\right) e^{i\left(\Delta \mathbf{r}_{a} \cdot \mathbf{k}_{a}-\Delta \mathbf{r}_{b} \cdot \mathbf{k}_{b}\right)} d^{2} \Delta \mathbf{r}_{a} d^{2} \Delta \mathbf{r}_{b}
$$

Informally, we may think of $P\left(\mathbf{r}_{b}, \mathbf{k}_{b} ; \mathbf{r}_{a}, \mathbf{k}_{a}\right)$ as the scattering response across space and wavevector at the output plane of optical rays at the input plane. For instance, if the single ray enters the system at $\mathbf{r}_{a}=0$ with $\mathbf{k}_{a}=0$, then the spatio-angular response at the target plane is given by $W_{b}\left(\mathbf{r}_{b}, \mathbf{k}_{b}\right)=P\left(\mathbf{r}_{b}, \mathbf{k}_{b} ; 0,0\right)$. Since it is not possible to form a single-ray input WDF, this remains an informal interpretation [11]. Although $P$ is a function of 4 variables, it only depends upon the scattering system's 2-variable transmission matrix, $T$, and obeys the same properties as a WDF (e.g., realness).

In Eq. (3.5), we recognize a Fourier transform from $\Delta \mathbf{r}_{b}$ to $\mathbf{k}_{b}$ and an inverse Fourier transform from $\Delta \mathbf{r}_{a}$ to $\mathbf{k}_{a}$. Performing the reversed transforms on both sides of Eq. (3.5) yields,

$$
T\left(\mathbf{r}_{b}^{+}, \mathbf{r}_{a}^{+}\right) T^{*}\left(\mathbf{r}_{b}^{-}, \mathbf{r}_{a}^{-}\right)=\frac{1}{(2 \pi)^{4}} \iint P\left(\mathbf{r}_{b}, \mathbf{k}_{b} ; \mathbf{r}_{a}, \mathbf{k}_{a}\right) e^{i\left(-\Delta \mathbf{r}_{a} \cdot \mathbf{k}_{a}+\Delta \mathbf{r}_{b} \cdot \mathbf{k}_{b}\right)} d^{2} \mathbf{k}_{a} d^{2} \mathbf{k}_{b}
$$

which can be inserted into Eq. (3.2) to arrive at our central result:

$$
\begin{array}{r}
C\left(\Delta \mathbf{r}_{b}, \Delta \mathbf{k}_{b} ; \Delta \mathbf{r}_{a}, \Delta \mathbf{k}_{a}\right)=\frac{1}{(2 \pi)^{4}} \iiint \int\left\langle P\left(\mathbf{r}_{b}, \mathbf{k}_{b} ; \mathbf{r}_{a}, \mathbf{k}_{a}\right)\right\rangle e^{i\left(-\Delta \mathbf{r}_{a} \cdot \mathbf{k}_{a}+\Delta \mathbf{r}_{b} \cdot \mathbf{k}_{b}\right)} \\
e^{i\left(\Delta \mathbf{k}_{a} \cdot \mathbf{r}_{a}-\Delta \mathbf{k}_{b} \cdot \mathbf{r}_{b}\right)} d^{2} \mathbf{k}_{a} d^{2} \mathbf{r}_{a} d^{2} \mathbf{k}_{b} d^{2} \mathbf{r}_{b} .
\end{array}
$$

In short, $C$ and the ensemble averaged $P$ are connected through two forward and two inverse 2D Fourier transforms. The correlation function in Eq. (3.7) is the formulation of our new generalized memory effect, generalizing the well-known 'tilt' memory effect into a full class of interrelated shift and tilt correlations. If $\langle P\rangle$ were to be separable in $\mathbf{r}$ and $\mathbf{k}$ coordinates, Eq. (3.7) would reduce to a simple multiplication of the 'tilt' and 'shift' memory effects. However, as we will show below, $\langle P\rangle$ is generally not separable, and the interaction between shift and tilt effects is non trivial. At this point we want to emphasize that Eq. (3.7) is still valid for any scattering medium or geometry. Also, these spatio-angular correlations are an intrinsic property of the scattering medium and will thus be present regardless of the form of the input field.

\subsection{Approximate solution for forward scattering}

We are now left with the challenge of calculating $\langle P\rangle$. Although we could use the radiative transfer equation in the most general case, we prefer an approximate solution that gives a simple analytical form for $\langle P\rangle$ under the condition that light is mainly scattered in the forward direction. 
The model is based on the notion that the WDF's joint description of light across $\mathbf{r}$ and $\mathbf{k}$ is very closely related to the light field description of rays [13]. Similar to the approach presented in Ref. [14], light propagation in the sample is modeled as a series of scattering events, where each scattering event slightly changes the propagation direction of the rays while maintaining the position. Between the scattering events, light propagates along straight rays and maintains its directionality.

In the continuous limit of infinitely small steps between the scattering events, this picture translates to a Fokker-Planck equation with the following solution (full derivation in the Appendix 3.7.2):

$$
P^{F P}(\hat{\mathbf{r}}, \hat{\mathbf{k}})=\frac{12 \ell_{t r}^{2}}{k_{0}^{2} L^{4}} \exp \left(-\frac{6 \ell_{t r}}{L}\left[\frac{|\hat{\mathbf{r}}|^{2}}{L^{2}}-\frac{\hat{\mathbf{k}} \cdot \hat{\mathbf{r}}}{k_{0} L}+\frac{|\hat{\mathbf{k}}|^{2}}{3 k_{0}^{2}}\right]\right) .
$$

Here, $\hat{\mathbf{k}} \equiv \mathbf{k}_{b}-\mathbf{k}_{a}, \hat{\mathbf{r}} \equiv \mathbf{r}_{b}-\mathbf{r}_{a}-L \mathbf{k}_{a} / k_{0}, k_{0}$ is the wavenumber, $\ell_{t r}$ is the transport mean free path, and $L$ is the separation between the input and target plane (that is, the target plane depth). It can be seen that the variance in the direction of the light increases linearly with $L$. Interestingly, in this forward scattering regime, the variance in the spatial distribution increases as $L^{3}$.

Note that $P^{F P}$ is only a function of two variables. The reduction of $\mathbf{r}_{b}$ and $\mathbf{r}_{a}$ to a single difference coordinate $\hat{\mathbf{r}}$ is possible because the Fokker-Planck equation is shift invariant. A similar simplification was used in the original derivation of the 'tilt' optical memory effect [6]. By assuming the average scattered intensity envelope only depended upon relative position, the resulting memory effect correlation reduced to a function of only one tilt variable, which is now a commonly applied simplification in many experiments [1-4]. Note that in this paraxial model the target intensity distribution is additionally offset by $L \mathbf{k}_{a} / k_{0}$, which is exactly what is expected from pure ballistic propagation through a transparent medium of thickness $L$. Moreover, the Fokker-Planck model is also invariant to a tilt in the incident wave. This symmetry allows for the reduction of coordinates $\mathbf{k}_{b}$ and $\mathbf{k}_{a}$ to $\hat{\mathbf{k}}$. Of course, this approximation neglects the fact that rays at a high incident angle propagate a larger distance inside the sample.

We can now find an expression for the generalized memory effect in a forward scattering material by substituting $\langle P\rangle=P^{F P}$ into Eq. (3.7) (see Appendix 3.7.2 for the details), arriving at

$$
C\left(\Delta \mathbf{r}_{b}, \Delta \mathbf{k}_{b} ; \Delta \mathbf{r}_{a}, \Delta \mathbf{k}_{a}\right)=(2 \pi)^{2} C^{F P}\left(\Delta \mathbf{r}_{b}, \Delta \mathbf{k}_{b}\right) \boldsymbol{\delta}\left(\Delta \mathbf{k}_{a}-\Delta \mathbf{k}_{b}\right) \delta\left(\Delta \mathbf{r}_{b}-\Delta \mathbf{r}_{a}-\Delta \mathbf{k}_{a} L / k_{0}\right) .
$$

Here the correlation function $C^{F P}$ is given by

$$
C^{F P}\left(\Delta \mathbf{r}_{b}, \Delta \mathbf{k}_{b}\right)=\exp \left(-\frac{L^{3} k_{0}^{2}}{2 \ell_{t r}}\left[\frac{\left|\Delta \mathbf{k}_{b}\right|^{2}}{3 k_{0}^{2}}-\frac{\Delta \mathbf{k}_{b} \cdot \Delta \mathbf{r}_{b}}{k_{0} L}+\frac{\left|\Delta \mathbf{r}_{b}\right|^{2}}{L^{2}}\right]\right) .
$$

As is clear from Eq. (3.10), shift and tilt correlations along the target plane are not independent but show a combined effect, represented by the $\Delta \mathbf{k}_{b} \cdot \Delta \mathbf{r}_{b}$ cross term. The two delta functions in Eq. (3.9) are a direct result of the shift and tilt invariance of the Fokker-Planck model. As we show in Appendix 3.7.3, in an actual experiment the delta functions will be replaced by the ambiguity function of the incident field, which is ideally a well-behaved sharp function. 


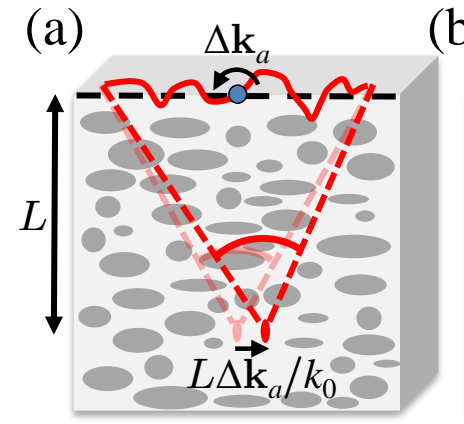

(b)

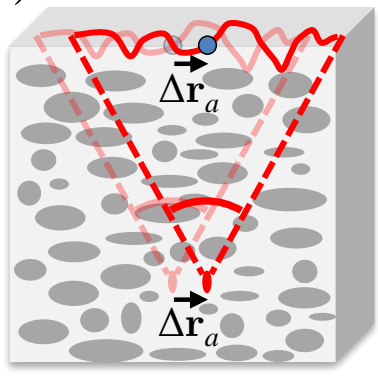

(c)

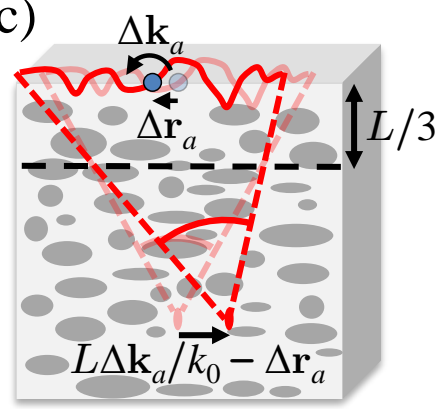

Figure 3.2: AO focus scanning/imaging inside a scattering medium uses different memory effects. (a) The 'tilt' effect arises with the AO tilt plane (dashed line) conjugated to the input surface. (b) The 'shift' effect arises with the AO tilt plane at infinity. (c) The optimal joint tilt/shift scheme requires the AO tilt plane to be located at a depth of $L / 3$ inside the sample.

\subsection{Maximizing the isoplanatic patch}

We now examine the application of the generalized memory effect to adaptive optics (AO) systems. AO allows light to be focused inside scattering media by correcting for the induced wavefront distortions by means of a deformable mirror or spatial light modulator. Generally, AO systems are limited to a single plane of wavefront correction. However, a single correction plane cannot correct for a full scattering volume and therefore the focus scan range is limited to a small area termed the isoplanatic patch. A central question in AO is where to conjugate the correction plane to maximize the isoplanatic patch [10]. In the case of conjugate $\mathrm{AO}$, this correction plane is located at the sample's top (input) surface, whereas in pupil $\mathrm{AO}$ it is effectively located at an infinite distance from the sample. In Fig. 3.2, we diagram how conjugate $\mathrm{AO}$ and pupil $\mathrm{AO}$ are analogous to experiments that utilize the 'tilt' (Fig. 3.2a) and 'shift' memory effects (Fig. 3.2b), respectively.

To maximize the isoplanatic patch, one should simultaneously tilt and shift the incident field to maximize $C^{F P}$ for a desired shift distance $\Delta \mathbf{r}_{b}$, while using $\Delta \mathbf{k}_{b}$ as a free parameter. From Eq. (3.10), we find that the optimal scan range is achieved when

$$
\Delta \mathbf{k}_{b}^{o p t}=\frac{3 k_{0} \Delta \mathbf{r}_{b}}{2 L}
$$

Likewise, $C^{F P}$ can also be expressed as a function of the translation $\Delta \mathbf{r}_{a}$ and rotation $\Delta \mathbf{k}_{a}$ of the incident field. Using the delta function relations from Eq. (3.9), we can substitute $\Delta \mathbf{r}_{b}=\Delta \mathbf{r}_{a}+L \Delta \mathbf{k}_{a} / k_{0}$ and $\Delta \mathbf{k}_{b}=\Delta \mathbf{k}_{a}$ in Eq. (3.11) to find the optimal tilt/shift combination at the input plane for a given amount of target shift $\Delta \mathbf{r}_{b}$ :

$$
\Delta \mathbf{k}_{a}^{o p t}=\frac{3 k_{0} \Delta \mathbf{r}_{b}}{2 L} \text { and } \Delta \mathbf{r}_{a}^{o p t}=-\Delta \mathbf{r}_{b} / 2
$$

Hence, to scan a focus by a distance of $\Delta \mathbf{r}_{b}$, the optimal strategy is to shift the incident field by $\Delta \mathbf{r}_{b} / 2$ in the opposite direction and then tilt until the desired $\Delta \mathbf{r}_{b}$ is reached. In other words, the optimal scanning mechanism is achieved by conjugating the $\mathrm{AO}$ correction plane 
Table 3.1: Comparison of the performance of the three different memory effects in terms of adaptive optics scan range.

\begin{tabular}{|l|l|c|c|}
\hline Correlation & Adaptive Optics & Tilt plane & Scan range \\
\hline Shift & Pupil & $-\infty$ & $\sqrt{2 \ell_{t r} / k_{0}^{2} L}$ \\
Tilt & Surface Conjugate & 0 & $\sqrt{6 \ell_{t r} / k_{0}^{2} L}$ \\
Optimal & Sample Conjugate & $L / 3$ & $\sqrt{8 \ell_{t r} / k_{0}^{2} L}$ \\
\hline
\end{tabular}

inside the scattering sample at a depth of $L / 3$, which geometrically corresponds to the ideal tilt plane. We illustrate this mechanism for optimized focus scanning in Fig. 3.2c.

In Table 3.1, we compare the isoplanatism provided by the three different memory effects. Here, we define isoplanatism as the maximum allowed scan distance $\mathbf{r}_{b}$ at the target plane that maintains $1 / e$ correlation, which we solve for with appropriately defined input variables in Eq. (3.10). Interestingly, all memory effects decrease at the same rate of $\sqrt{\ell_{t r} / k_{0}^{2} L}$ as the target plane is placed deeper inside the medium. However, the scan range of the tilt/tilt memory effect is always a factor of $\sqrt{3}$ larger than the shift/shift memory effect. By exploiting the optimal tilt/shift combination, as given in Eq. (3.12), the scanning improvement is increased to a factor of 2 .

For some applications, such as imaging a structure hidden far behind a forward scattering material $[1-3,15]$, one may be interested in maximizing the angular memory effect instead. In this case, the goal is to maximize $C^{F P}$ for a desired tilt $\Delta \mathbf{k}_{b}$ while using $\Delta \mathbf{r}_{b}$ as a free parameter. For this scenario, we find a maximum scan range of $\Delta k_{b}=\sqrt{24 \ell_{t r} / L^{3}}$ for $\Delta x_{b}=\Delta k_{b} L / 2 k_{0}$. This translates to a $1 / e$ memory effect angle of $\Delta k_{b} / k_{0} \approx 0.78 \sqrt{\frac{\ell_{t r}}{L}} \frac{\lambda}{L}$. Of course, these results are based on the Fokker-Planck model, which is only valid in the forward scattering regime $L<\ell_{t r}$. In this regime, however, the memory effect angle is significantly larger than the $1 / e$ angle of $0.43 \lambda / L$ that follows from the tilt/tilt memory effect [6] alone. This enhanced angular memory effect is in line with recent observations in forward scattering tissue [8].

\subsection{Experimental validation}

We now measure $C$ and $P$ for a forward scattering medium in two separate experiments. These experiments will verify the $C-P$ relation predicted in Eq. (3.7) and the Fokker-Planck model in Eq. (3.8). For simplicity, we consider $C$ and $P$ only along the horizontal dimension, with $\mathrm{x}_{b}$ and $\mathrm{k}_{b}$ as the horizontal components of the position and the wavevector at the sample back surface, respectively. We created our scattering samples using uniform $3.17 \pm 0.32 \mu \mathrm{m}$ diameter silica microspheres immersed in $1 \%$ agarose gel with a concentration of $1.50 \pm$ $0.01 \cdot 10^{-4}$ spheres per $\mu \mathrm{m}^{3}$. The refractive index of the microspheres and the agarose gel are $1.45 \pm 0.02$ and $1.33 \pm 0.005$, respectively. Using Mie theory, we find an anisotropy factor of $g=0.980 \pm 0.007$ and a scattering mean free path of $\ell_{s c}=0.296 \pm 0.016 \mathrm{~mm}$ at a wavelength of $632.8 \mathrm{~nm}$. This results in a transport mean free path of $\ell_{t r}=14.8 \pm 5.2 \mathrm{~mm}$. We then cast this scattering mixture into layers of 2 thicknesses: $L=258 \pm 3 \mu \mathrm{m}$ and $520 \pm 5 \mu \mathrm{m}$.

The experimental setup is illustrated in Fig. 3.3. Light from a $632.8 \mathrm{~nm} \mathrm{HeNe}$ laser is 


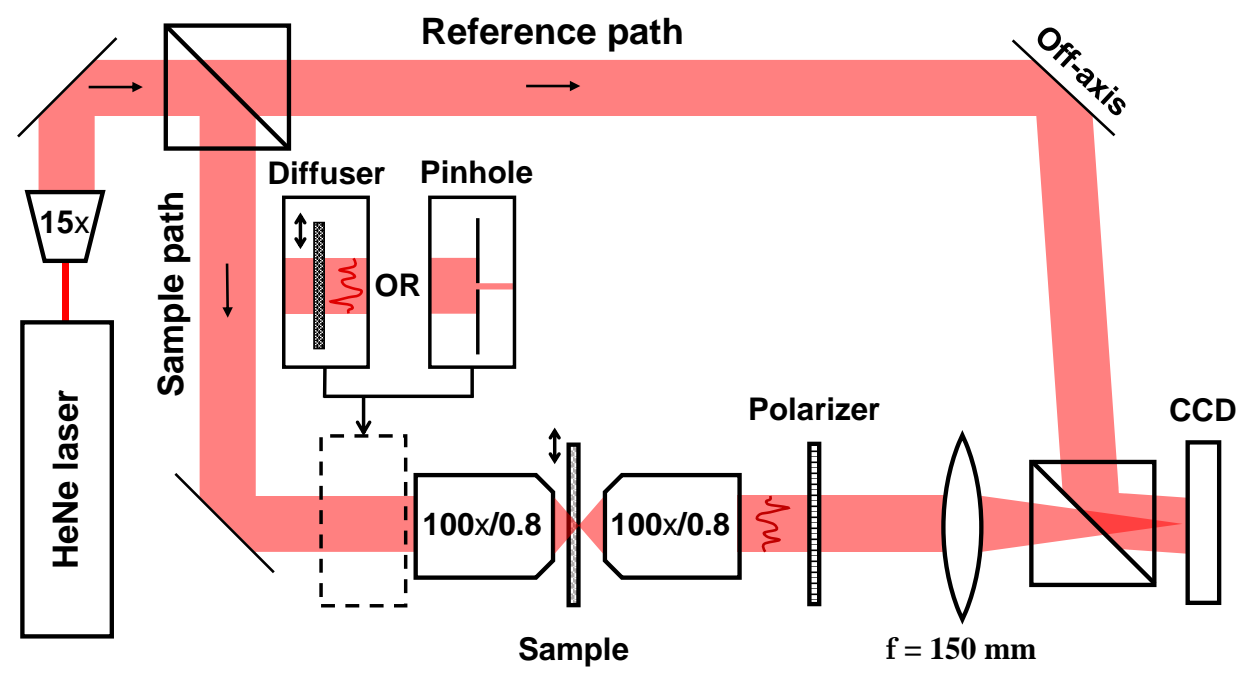

Figure 3.3: Schematic of the experimental setup used for measuring both the light field transmission function and generalized correlation functions. The pinhole and diffuser are used in the $P$ and $C$ experiments, respectively. Both the diffuser and the sample holder are placed on a translation stage.

expanded and split into a reference path and the sample path. Light is focused onto the scattering sample using a microscope objective (Zeiss A-Plan 100x/0.8), and we image the sample backsurface (target plane) with a CCD camera (AVT Stingray F-145) through a second identical objective and tube lens $(f=150 \mathrm{~mm}$ ). The phase of light transmitted through the sample is determined by means of off-axis holography. The sample is placed on a translation stage for lateral movement. Additionally, a pinhole or diffuser is placed directly in front of the first microscope objective to either create a pencil beam or a random speckle pattern as input field.

\subsubsection{Measurements of the light field transmission function}

From Eq. (3.4), we know that a finite input beam $E_{a}$ will result in a WDF at the target plane, $W_{b}$, that is a convolution between the desired light field transmission function $P$ and the WDF of the input field, $W_{a}$. We are thus able to determine $P$ by using a pencil beam for sample illumination (with a size of $2.0 \mu \mathrm{m}$ ) at $\mathrm{x}_{a}=0$ and $\mathrm{k}_{a}=0$, which approximately forms the input WDF: $W_{a}\left(\mathrm{x}_{a}, \mathrm{k}_{a}\right) \propto \delta\left(\mathrm{x}_{a}\right) \delta\left(\mathrm{k}_{a}\right)$. $P$ is then found by measuring the scattered light at the target plane across both space and angle. The pencil beam is formed by placing a $500 \mu \mathrm{m}-$ wide pinhole close to the back-aperture of the first objective. After measuring the scattered field, we numerically calculate the WDF $W_{b}\left(\mathrm{x}_{b}, \mathrm{k}_{b}\right)$, using Eq. (3.3), to find the scattered intensity as a function of both $\mathrm{x}_{b}$ and $\mathrm{k}_{b}$. We average the WDF over 300 measurements, translating the sample over a distance of $10 \mu \mathrm{m}$ in between each measurement to obtain $\langle P\rangle$. To facilitate comparison of our measurements with the Fokker-Planck model, which does not include ballistic light, we chose to remove the contribution of ballistic light by subtracting the average transmitted field from every measured field before calculating its WDF. Following 


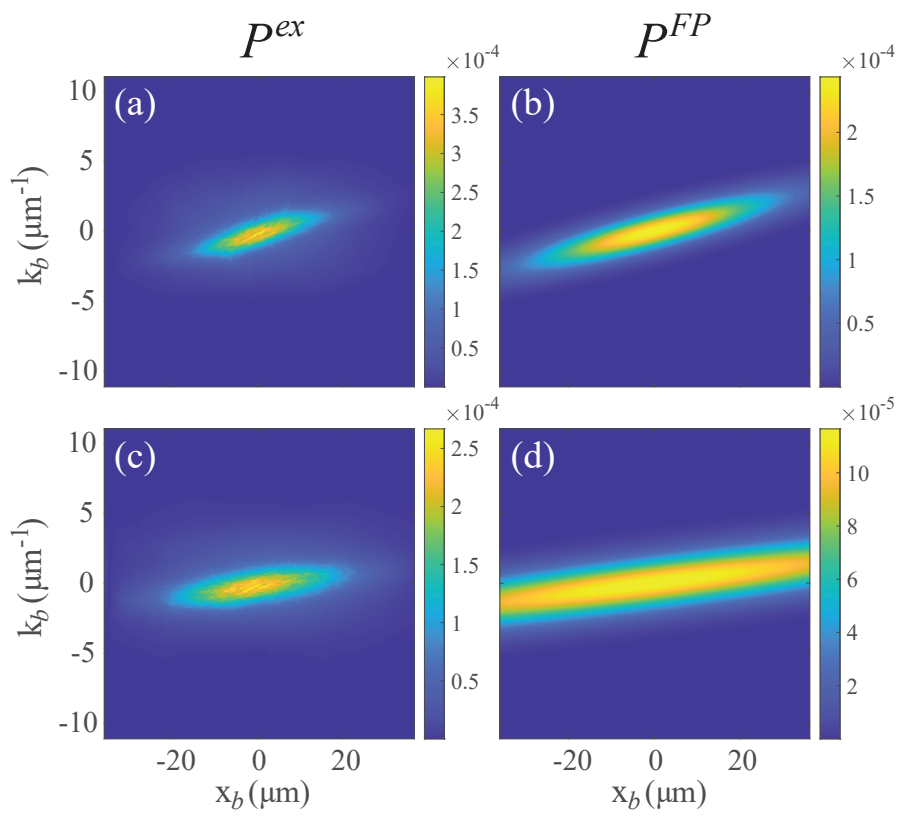

Figure 3.4: Results of the light field transmission function $(P)$ experiment. We compare our measurements, $P^{e x}$, to the Fokker-Planck model prediction $\left(P^{F P}\right.$, from Eq. (3.8)) for samples with (a,b) $L=258 \mu \mathrm{m}$ and (c,d) $L=520 \mu \mathrm{m}$. Colorbar indicates the normalized transmitted intensity as function of $\mathrm{x}_{b}$ and $\mathrm{k}_{b}$.

prior work, it is possible to modify our model to also include ballistic light at the expense of some added complexity $[16,17]$.

In Fig. 3.4, we compare our measured light field transmission functions, $P^{e x}$, to those computed with the Fokker-Plank model $\left(P^{F P}\right)$ in Eq. (3.8). The effect of the cross-term between $\mathrm{x}_{b}$ and $\mathrm{k}_{b}$, as predicted in $P^{F P}$, is clearly visible in our measurements from the fact that the distributions are sheared. This shear implies that the light at the edges of the diffuse spot (large $\mathrm{x}_{b}$ ) continues to propagate, on average, in a radially outward manner (large $\mathrm{k}_{b}$ ) after scattering. The $P^{e x}$ measurements are less spread out than the Fokker-Planck model, which might be a result of the limited optical sensitivity at the edges of the objective lenses. Both a large field of view and a large numerical aperture of the microscope objective are required to measure the full extent of the light field transmission function.

\subsubsection{Measurements of the generalized correlation function}

Next, we experimentally measure the generalized correlation function $C^{e x}$. We will directly compare these measurements to our correlation model for $C^{F P}$ in Eq. (3.10). Additionally, we will verify our main result in Eq. (3.7) by comparing the $C^{e x}$ measurements to $C^{P W}$, the $2 \mathrm{D}$ Fourier transform of the light field transmission function measurements. For the correlation measurements, we replace the pinhole in front of the first objective with a diffuser that forms a randomized input field (average speckle size $=400 \mathrm{~nm}$ ) at the sample surface. We use a diffuser to minimize correlations within the input field, which manifest themselves as a 
convolution in our measurement of $C^{e x}$ (see Appendix 3.7.3). We tilt the random input field by translating the diffuser at the objective back aperture. We record a total of 625 scattered fields by illuminating the sample at 25 unique spatial locations and under 25 unique angles of incidence. From this $25^{2}$ data cube, we compute $C^{e x}$ by taking the ensemble average of all the absolute values of the correlation coefficients between two fields separated by the same amount of shift $\Delta \mathrm{x}_{b}$ and tilt $\Delta \mathrm{k}_{b}$. Finally, we normalize $C^{e x}$ after subtracting the correlation value at the maximum shift, corresponding with the correlations in the ballistic light.

Fig. 3.5 presents the results of our $C$ experiments. For the two different sample thicknesses of $L=258 \mu \mathrm{m}$ and $L=520 \mu \mathrm{m}$, the measured 2D correlation functions in (a,d) are compared to $C^{P W}$, the Fourier transform of $P^{e x}$ in $(\mathrm{b}, \mathrm{e})$, and the Fokker-Planck model $C^{F P}$ in $(\mathrm{c}, \mathrm{f})$. The dashed line indicates the optimal tilt/scan condition as predicted in Eq. (3.11). We also show cross sections through $C^{e x}$ (red stars), $C^{P W}$ (blue stars) and $C^{F P}$ (dashed black) on the right. These cross sections are given along the $\Delta \mathrm{k}_{b}=0$ line $(\mathrm{g}, \mathrm{j})$, the $\Delta \mathrm{x}_{b}=0$ line $(\mathrm{h}, \mathrm{k})$, and the optimal target plane scan condition (i,l). These last two plots demonstrate how jointly considered tilts and shifts can increase correlations along $\Delta \mathrm{x}_{b}$ to increase the scan range (i.e., isoplanatic patch) at the target plane. For instance, for the $258 \mu \mathrm{m}$-thick sample, the standard deviation increases from $1.30 \mu \mathrm{m}$ to $2.66 \mu \mathrm{m}$ when comparing the correlation function along $\Delta \mathrm{x}_{b}$ and $\Delta \mathrm{k}_{b}^{o p t}$, which corresponds to the predicted doubling of the scan range. Even though the Fokker-Planck model is a simplified paraxial description that neglects backscattering and interference, it provides an accurate prediction of the measured correlations. The cross sections for $C^{e x}$ and $C^{P W}$ are also in good agreement, except for Fig. 3.5k. This data corresponds to the thickest sample, for which the limited field of view of the objective lens prevented us from fully measuring $P^{e x}$ along $\mathrm{x}_{b}$. The truncation of $P^{e x}$, already observed in Fig. 3.4c, results in a broadening of its Fourier transform $C^{P W}$.

\subsection{Discussion}

The optical memory effect as reported in Ref. [6] has paved the way for several new imaging techniques that can 'see through' thin scattering layers [1-4]. These techniques require the object of interest to be positioned at a distance behind the thin layer and are thus not immediately applicable to situations in biomedical imaging where the object of interest is embedded in scattering tissue. The anisotropic memory effect [9] showed that translational spatial correlations also exists within the scattering sample, which have been demonstrated to be suitable for imaging or focus scanning inside biological tissue $[15,18]$. Our new generalized memory effect model offers a new theoretical framework that encompasses the two known memory effects as special cases. Moreover, our model offers a means to optimize the field of view of adaptive optics and hidden imaging approaches.

Using the Fokker-Planck light propagation model, we have shown that the 'tilt' memory effect is not only present behind, but also inside scattering layers, proving to be a factor $\sqrt{3}$ more effective as a scanning technique than the 'shift' memory effect alone. This finding supports the field-of-view (FOV) advantage of conjugate AO over pupil AO discussed in Ref. [10], as the 'tilt' and 'shift' memory effects are utilized by conjugate AO and pupil $\mathrm{AO}$, respectively. Our optimal joint tilt/shift scheme, which corresponds to an optimal AO conjugation tilt plane, maximizes the corrected FOV beyond what is predicted independently by only the 'tilt' memory effect and only the 'shift' memory effect. The scan ranges given in 

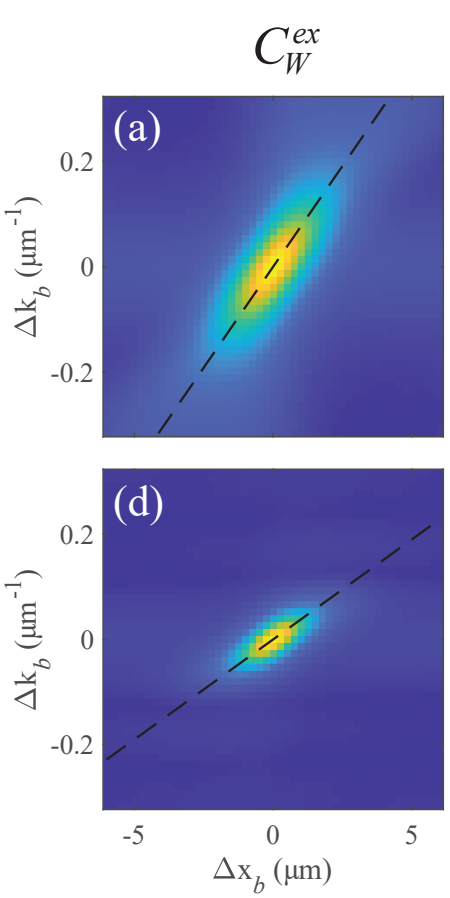

$C_{W}^{P W}$
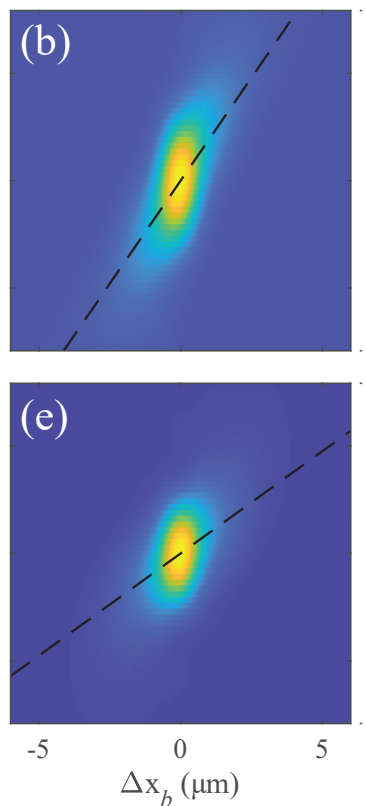
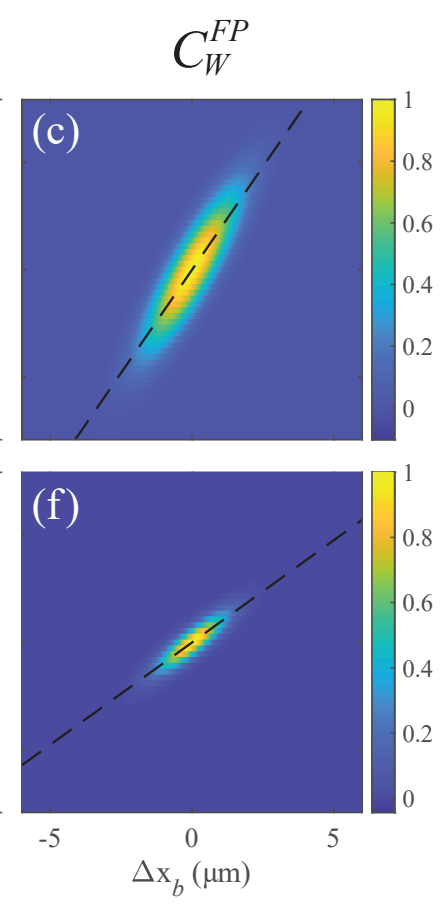
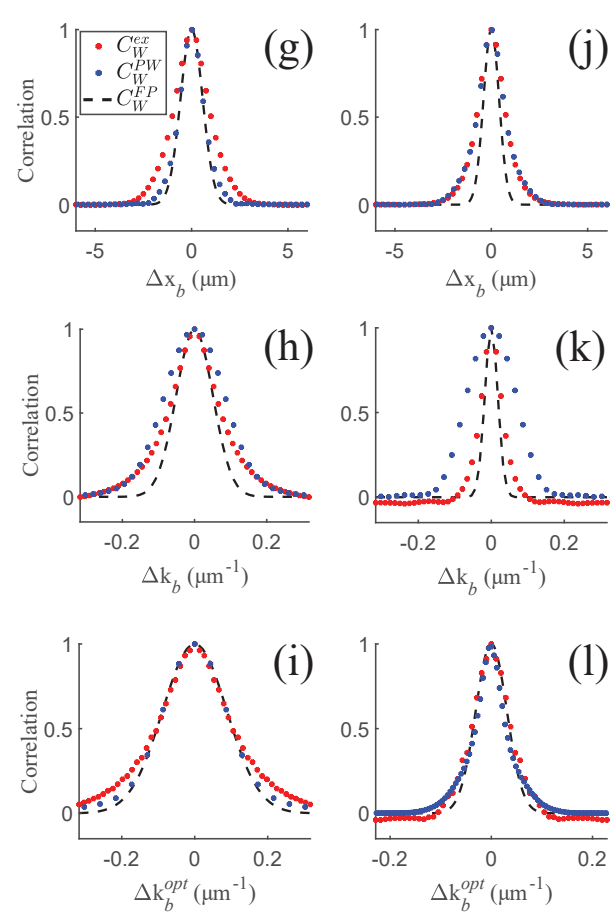

Figure 3.5: Results of the generalized correlation function $(C)$ experiments. Measurements of $C^{e x}$ are compared to $C^{P W}$ and the FokkerPlanck correlation model $C^{F P}$ from Eq. (3.10) for samples with (a)-(c) $L=258 \mu \mathrm{m}$ and (d)-(f) $L=520 \mu \mathrm{m}$. Dashed lines indicate the optimal scanning condition in Eq. (3.11). (g)-(i) Cross sections of the 2D correlation functions in (a)-(c), evaluated (g) along the horizontal axis at $\Delta \mathrm{k}_{b}=0$, (h) along vertical axis at $\Delta \mathrm{x}_{b}=0$, and (i) along the optimal scan line for the sample with $L=258 \mu \mathrm{m}$. (j)-(l) Same cross sections for the sample with $L=520 \mu \mathrm{m}$. The dashed line denotes the $C^{F P}$ model. The red and blue stars denote the measured $C^{e x}$ and $C^{P W}$. 
Table 3.1 are strictly the correlations that result from the transmission matrix. Correlations in the input beam may further extend the scan range of the different memory effects (see Appendix 3.7.3). The Fokker-Planck model assumes continuous scattering throughout the sample and neglects back-scattered light, an assumption that may not hold in strong isotropic scattering media. However, in this case the Fourier relation between $P$ and $C$ is still valid. The generalized correlation function is the phase-space equivalent of the $C_{1}$ intensity correlations introduced by Feng et al. [6]. We envisage that there also exist phase-space equivalents of $C_{2}$ and $C_{3}$ correlations. These higher-order correlations have recently been exploited to focus light through scattering media with an unexpectedly high efficiency [19], and investigating their phase-space equivalents may prove equally useful.

Concluding, our new generalized memory effect model generalizes the known optical memory effects without making any assumptions about the geometry or scattering properties of the scattering system. The predicted Fourier relation between the light field transmission function and generalized correlation function has been experimentally verified in forward scattering media. Furthermore, we found that the simple Fokker-Planck model for light propagation is surprisingly accurate in describing the full set of first-order spatial correlations inside forward scattering media. Our new generalized memory effect model predicts the maximum distance that a scattered field can be scanned while remaining correlated to its unshifted form. In other words, the generalized memory effect provides the optimal scan mechanism for deep-tissue focusing techniques.

\subsection{Appendices}

\subsubsection{Wigner input-output derivation}

Here we derive the expression for the light field transmission function $P$ in Eq. (3.5), in terms of the transmission matrix $T$. We will find that $P$, as a 4D function, is given by a double Wigner transform of the 2D function $T$ [11]. To begin, we first substitute the definition of the transmission matrix from Eq. (3.1) into the definition of the WDF in Eq. (3.3). We chose to examine the WDF at the target plane along coordinates $\left(\mathbf{r}_{b}, \mathbf{k}_{b}\right)$ :

$$
\begin{aligned}
W_{b}\left(\mathbf{r}_{b}, \mathbf{k}_{b}\right) & =\int E_{b}\left(\mathbf{r}_{b}^{+}\right) E_{b}^{*}\left(\mathbf{r}_{b}^{-}\right) e^{-i \Delta \mathbf{r}_{b} \cdot \mathbf{k}_{b}} d^{2} \Delta \mathbf{r}_{b} \\
& =\int\left[\int T\left(\mathbf{r}_{b}^{+}, \mathbf{r}_{a}^{\prime}\right) E_{a}\left(\mathbf{r}_{a}^{\prime}\right) d^{2} \mathbf{r}_{a}^{\prime}\right]\left[\int T^{*}\left(\mathbf{r}_{b}^{-}, \mathbf{r}_{a}^{\prime \prime}\right) E_{a}^{*}\left(\mathbf{r}_{a}^{\prime \prime}\right) d^{2} \mathbf{r}_{a}^{\prime \prime}\right] e^{-i \Delta \mathbf{r}_{b} \cdot \mathbf{k}_{b}} d^{2} \Delta \mathbf{r}_{b} .
\end{aligned}
$$

Here, $\mathbf{r}_{a}^{\prime}$ and $\mathbf{r}_{a}^{\prime \prime}$ are unique spatial coordinates along the input plane and we again use the notation $\mathbf{r}_{b}^{+} \equiv \mathbf{r}_{b}+\Delta \mathbf{r}_{b} / 2, \mathbf{r}_{b}^{-} \equiv \mathbf{r}_{b}-\Delta \mathbf{r}_{b} / 2$. Without loss of generality, we may transform the variables $\mathbf{r}_{a}^{\prime}$ and $\mathbf{r}_{a}^{\prime \prime}$ into center-difference coordinates with the substitution $\mathbf{r}_{a}^{\prime}=\mathbf{r}_{a}^{+}$and $\mathbf{r}_{a}^{\prime \prime}=\mathbf{r}_{a}^{-}$to create,

$$
W_{b}\left(\mathbf{r}_{b}, \mathbf{k}_{b}\right)=\iiint T\left(\mathbf{r}_{b}^{+}, \mathbf{r}_{a}^{+}\right) T^{*}\left(\mathbf{r}_{b}^{-}, \mathbf{r}_{a}^{-}\right) E_{a}\left(\mathbf{r}_{a}^{+}\right) E_{a}^{*}\left(\mathbf{r}_{a}^{-}\right) e^{-i \Delta \mathbf{r}_{b} \cdot \mathbf{k}_{b}} d^{2} \Delta \mathbf{r}_{a} d^{2} \Delta \mathbf{r}_{b} d^{2} \mathbf{r}_{a} .
$$

By taking the inverse Fourier transform of Eq. (3.3) we find,

$$
E_{a}^{*}\left(\mathbf{r}_{a}^{-}\right) E_{a}\left(\mathbf{r}_{a}^{+}\right)=\frac{1}{(2 \pi)^{2}} \int W_{a}\left(\mathbf{r}_{a}, \mathbf{k}_{a}\right) e^{i \mathbf{k}_{a} \cdot \Delta \mathbf{r}_{a}} d^{2} \mathbf{k}_{a}
$$


Substituting this into Eq. (3.15) gives,

$$
\begin{aligned}
& W_{b}\left(\mathbf{r}_{b}, \mathbf{k}_{b}\right)=\frac{1}{(2 \pi)^{2}} \iint {\left[\iint\left\langle T\left(\mathbf{r}_{b}^{+}, \mathbf{r}_{a}^{+}\right) T^{*}\left(\mathbf{r}_{b}^{-}, \mathbf{r}_{a}^{-}\right)\right\rangle e^{i\left(\Delta \mathbf{r}_{a} \cdot \mathbf{k}_{a}-\Delta \mathbf{r}_{b} \cdot \mathbf{k}_{b}\right)} d^{2} \Delta \mathbf{r}_{a} d^{2} \Delta \mathbf{r}_{b}\right] } \\
& W_{a}\left(\mathbf{r}_{a}, \mathbf{k}_{a}\right) d^{2} \mathbf{r}_{a} d^{2} \mathbf{k}_{a} .
\end{aligned}
$$

In the term in brackets, we recognize the definition of $P$ in Eq. (3.5), where the light field transmission function is $P$ given as a double Wigner transform of the transmission matrix $T$. Substituting the definition of $P$ reduces Eq. (3.17) to Eq. (3.4). Finally, we note that $P$ may be equivalently defined through the k-space transmission matrix, or in any other basis, as long as the matrix offers a full description of the scattering system.

\subsubsection{Fokker-Planck model}

In this section, we derive a simple model for the light field transmission function $P$ for a forward scattering material. Following Ref. [14], we only consider the regime where the light is still propagating mainly in the forward direction, and we model the light propagation as a sequence of scattering events. For this model, we interpret the light field distribution $I(\mathbf{k}, \mathbf{r}, z)$ as a density of 'rays' at depth $z$ with lateral velocity $c \mathbf{k} / k_{0}$ and lateral position $\mathbf{r}$. Each scattering event randomly perturbs the direction of the light to some extent, whereas in between the scattering events light travels along straight trajectories in the direction $\mathbf{k} / k_{0}$. Importantly, the paraxial approximation dictates that the light propagates at a constant velocity in the positive $z$ direction, so that diffusion only occurs in the $x y$ plane. Clearly, this assumption is only valid when $|\mathbf{k}| \ll k_{0}$, i.e. in the paraxial limit.

This physical model is analogous to that of the propagation and collisions of particles in a gas, where the sequence of collisions and ballistic propagation gives rise to a special form of the Fokker-Planck equation known as the Kramers equation [20, 21]. Writing this Fokker-Planck equation for 2-dimensional diffusion gives

$$
\frac{\partial I(\mathbf{r}, \mathbf{k}, z)}{\partial z}=-\frac{k_{x}}{k_{0}} \frac{\partial I(\mathbf{r}, \mathbf{k}, z)}{\partial x}-\frac{k_{y}}{k_{0}} \frac{\partial I(\mathbf{r}, \mathbf{k}, z)}{\partial y}+q k_{0}^{2}\left(\frac{\partial^{2} I(\mathbf{r}, \mathbf{k}, z)}{\partial k_{x}^{2}}+\frac{\partial^{2} I(\mathbf{r}, \mathbf{k}, z)}{\partial k_{y}^{2}}\right) .
$$

Here, we omitted the terms responsible for dynamical friction and external forces, as they do not apply to diffusion of light. We used the paraxial approximation $z=c t$ to replace the usual dependency on time $t$ by a dependency on depth $z$. In this equation, the first two terms on the right hand side correspond to propagation of light along straight trajectories (similar to an advection equation), while the last two terms describe the change of propagation direction due to scattering. We will find an expression for the scattering strength $q$ later.

The model in Eq. (3.18) neglects all backscattered light. Furthermore, the use of a FokkerPlanck equation implies a continuous diffusion process, i.e., even after a very small propagation distance all light has been scattered, albeit at a very small angle. Physically, this model corresponds to having the limit of the scattering length approach zero, while keeping the transport length fixed. The important implication of this assumption is that there is no ballistic light in the Fokker-Planck model.

To solve this equation, we follow the general approach outlined in Ref. [22]. First, we note that Eq. (3.18) is separable in the $\left(x, k_{x}\right)$ and $\left(y, k_{y}\right)$ coordinates, so it is sufficient to solve 
only for the 1-dimensional equation

$$
\frac{\partial I(x, k, z)}{\partial z}=-\frac{k}{k_{0}} \frac{\partial I(x, k, z)}{\partial x}+q k_{0}^{2} \frac{\partial^{2} I(x, k, z)}{\partial k^{2}} .
$$

Second, we apply two Fourier transforms $(x \rightarrow \kappa$ and $k \rightarrow \xi)$ to transform the partial differential equation (PDE) from a second order PDE into a first order PDE

$$
\frac{\partial \tilde{I}(\kappa, \xi, z)}{\partial z}=\frac{\kappa}{k_{0}} \frac{\partial \tilde{I}(\kappa, \xi, z)}{\partial \xi}-q k_{0}^{2} \xi^{2} \tilde{I}(\kappa, \xi, z) .
$$

Next, we substitute $\tilde{I}=e^{Q}$ to further simplify the equation

$$
\frac{\partial Q(\kappa, \xi, z)}{\partial z}=\frac{\kappa}{k_{0}} \frac{\partial Q(\kappa, \xi, z)}{\partial \xi}-q k_{0}^{2} \xi^{2} .
$$

To solve this PDE, we use the method of characteristics. We parameterize $\xi=-\kappa s / k_{0}+$ $\xi_{0}, z=s$, and realize that

$$
\frac{d Q}{d s}=\frac{\partial Q}{\partial z} \frac{d z}{d s}+\frac{\partial Q}{\partial \xi} \frac{d \xi}{d s}=\frac{\partial Q}{\partial z}-\frac{\kappa}{k_{0}} \frac{\partial Q}{\partial \xi},
$$

which reduces Eq. (3.21) to an ordinary differential equation

$$
\frac{d Q}{d s}=-q k_{0}^{2} \xi^{2}=-q\left(-\kappa s+k_{0} \xi_{0}\right)^{2}=-q\left(\kappa^{2} s^{2}-2 \kappa k_{0} \xi_{0} s+k_{0}^{2} \xi_{0}^{2}\right) .
$$

For simplicity, we now first assume that the initial light field at $z=0$ is given by a 'ray' with normally incident ray at the origin: $I(x, k, 0)=2 \pi \delta(k) \delta(x)$. The Fourier transform of this delta function is a constant, so we require $Q(\kappa, \xi, 0)=C$. With this initial condition, we can now solve Eq. (3.23)

$$
\begin{aligned}
Q & =-q\left(\frac{1}{3} \kappa^{2} s^{3}-\kappa k_{0} \xi_{0} s^{2}+k_{0}^{2} \xi_{0}^{2} s\right)+C \\
& =-q\left(\frac{1}{3} \kappa^{2} z^{3}-\kappa k_{0} \xi z^{2}-\kappa^{2} z^{3}+k_{0}^{2} \xi^{2} z+2 \kappa k_{0} \xi z^{2}+\kappa^{2} z^{3}\right)+C \\
& =-q\left(\frac{1}{3} \kappa^{2} z^{3}+\kappa k_{0} \xi z^{2}+k_{0}^{2} \xi^{2} z\right)+C
\end{aligned}
$$

where we substituted back $\xi_{0}=\xi+\kappa s / k_{0}, s=z$ in the second step. So, $\tilde{I}=e^{Q}$ is a multivariate Gaussian distribution over $\xi$ and $\kappa$, where $C$ is chosen to normalize the distribution. We can finally write the solution as a multivariate Gaussian

$$
\tilde{I}=\frac{1}{2 \pi \sqrt{\left|\Sigma_{F}\right|}} \exp \left(-\frac{1}{2}\left[\begin{array}{l}
\kappa \\
\xi
\end{array}\right]^{T} \Sigma_{F}^{-1}\left[\begin{array}{l}
\kappa \\
\xi
\end{array}\right]\right),
$$

with the inverse covariance matrix

$$
\Sigma_{F}^{-1}=q z\left[\begin{array}{cc}
\frac{2 z^{2}}{3} & k_{0} z \\
k_{0} z & 2 k_{0}^{2}
\end{array}\right]
$$


Finally, we need to Fourier transform back to find $I$. To evaluate the inverse Fourier transforms, we simply invert the covariance matrix. In 2-dimensions we now find a covariance matrix of

$$
\Sigma=q z\left[\begin{array}{cccc}
\frac{2 z^{2}}{3} & 0 & k_{0} z & 0 \\
0 & \frac{2 z^{2}}{3} & 0 & k_{0} z \\
k_{0} z & 0 & 2 k_{0}^{2} & 0 \\
0 & k_{0} z & 0 & 2 k_{0}^{2}
\end{array}\right], \quad \Sigma^{-1}=\frac{3}{q z}\left[\begin{array}{cccc}
\frac{2}{z^{2}} & 0 & -\frac{1}{k_{0} z} & 0 \\
0 & \frac{2}{z^{2}} & 0 & -\frac{1}{k_{0} z} \\
-\frac{1}{k_{0} z} & 0 & \frac{2}{3 k_{0}^{2}} & 0 \\
0 & -\frac{1}{k_{0} z} & 0 & \frac{2}{3 k_{0}^{2}} .
\end{array}\right] .
$$

So far, we only considered normally incident rays at the origin. However, by introducing difference coordinates $\hat{\mathbf{k}} \equiv \mathbf{k}-\mathbf{k}_{a}$ and $\hat{\mathbf{r}} \equiv \mathbf{r}-\mathbf{r}_{a}-L \mathbf{k}_{a} / k_{0}$ and repeating the derivation above, we find the general solution for the general initial condition $I(\mathbf{r}, \mathbf{k}, 0)=(2 \pi)^{2} \boldsymbol{\delta}\left(\mathbf{k}-\mathbf{k}_{a}\right) \boldsymbol{\delta}(\mathbf{r}-$ $\left.\mathbf{r}_{a}\right)$, i. e. for any incident 'ray' $\left(\mathbf{r}_{a}, \mathbf{k}_{a}\right)$. This brings us to the final result

$$
I(\hat{\mathbf{r}}, \hat{\mathbf{k}}, z)=\frac{3}{k_{0}^{2} q^{2} z^{4}} \exp \left(-\frac{3}{q z}\left[\frac{|\hat{\mathbf{r}}|^{2}}{z^{2}}-\frac{\hat{\mathbf{r}} \cdot \hat{\mathbf{k}}}{z k_{0}}+\frac{|\hat{\mathbf{k}}|^{2}}{3 k_{0}^{2}}\right]\right) .
$$

It can be easily verified through substitution that Eq. (3.30) is a (normalized) solution to Eq. (3.18). The difference coordinate variables $\hat{\mathbf{k}}$ and $\hat{\mathbf{r}}$ now define the two-dimensional light field transmission function $P^{F P}(\hat{\mathbf{r}}, \hat{\mathbf{k}}) \equiv I(\hat{\mathbf{r}}, \hat{\mathbf{k}}, L)$ that we introduced in Eq. (3.8). It is worth noting that an identical result is found by modeling the sample as a stack of infinitely thin phase plates with free-space propagation between the plates, and then taking the limit of vanishing distance between the phase plate. This approach was introduced by Liu et al. [14] to calculate the average Wigner function for a point source embedded inside a scattering medium.

At this point, we still need to determine the scattering strength $q$. In order to do so, we compare our results to a discrete scattering model, which assumes that light propagates an average distance of $\ell_{s c}$ between each scattering event (i.e., $\ell_{s c}$ is the medium's scattering mean free path).

For this comparison, we first use Eq. (3.30) to calculate the average angular spread of light, $\left\langle|\hat{\mathbf{k}}|^{2}\right\rangle$, as a function of depth:

$$
\left\langle|\hat{\mathbf{k}}|^{2}\right\rangle \equiv \frac{1}{(2 \pi)^{2}} \iint|\hat{\mathbf{k}}|^{2} I(\hat{\mathbf{r}}, \hat{\mathbf{k}}, z) d \hat{\mathbf{r}} d \hat{\mathbf{k}}=4 k_{0}^{2} z q .
$$

Here, we see that the variance of the angular distribution grows linearly with propagation distance $z$, consistent with a diffusion process in the $\mathbf{k}_{b}$ coordinate system at the target plane.

Second, we calculate the expected angular spread as a function of depth for the discrete scattering model. After propagating a short distance $z$, a fraction of $1-\exp \left(z / \ell_{s c}\right)$ of the light is scattered. The angular spread of the scattered light is usually quantified with the anisotropy factor $g \equiv\langle\cos \theta\rangle$, with $\theta$ the scattering angle. Of course, the non-scattered light will still maintain its original direction. When $z$ is small, we can write $g=\langle\cos \theta\rangle \approx 1-\left\langle\left|\mathbf{k}_{b}\right|^{2}\right\rangle /\left(2 k_{0}^{2}\right)$ and we find that

$$
\left\langle|\hat{\mathbf{k}}|^{2}\right\rangle=2 k_{0}^{2}\left(1-e^{-z / \ell_{s c}}\right)(1-g) \approx 2 k_{0}^{2} z \frac{1-g}{\ell_{s c}} .
$$

In the limit of $z \rightarrow 0$, we can combine Eq. (3.31) and Eq. (3.32) to find

$$
q=\frac{1-g}{2 \ell_{s c}}=\frac{1}{2 \ell_{t r}},
$$


with $\ell_{t r} \equiv \ell_{s c} /(1-g)$ denoting the transport mean free path. Substituting this result into Eq. (3.30) finally gives us Eq. (3.10) in the main text.

To find $C^{F P}$, we need to evaluate Eq. (3.7) using the solution for $\langle P\rangle=P^{F P}$ in Eq. (3.10). Since this solution only depends on the difference coordinates $\hat{\mathbf{r}}$ and $\hat{\mathbf{k}}$, we first rewrite the integrals over $\mathbf{r}_{b}$ and $\mathbf{k}_{b}$ to integrals over $\hat{\mathbf{r}}$ and $\hat{\mathbf{k}}$ by applying the following changes of variables: $\mathbf{r}_{b} \rightarrow \hat{\mathbf{r}}+\mathbf{r}_{a}+L \mathbf{k}_{a} / k_{0}$ and $\mathbf{k}_{b} \rightarrow \hat{\mathbf{k}}+\mathbf{k}_{a}$. Doing so leads to,

$$
\begin{aligned}
C\left(\Delta \mathbf{r}_{b}, \Delta \mathbf{k}_{b} ; \Delta \mathbf{r}_{a}, \Delta \mathbf{k}_{a}\right)= & \frac{1}{(2 \pi)^{4}} \iiint \int P^{F P}(\hat{\mathbf{r}}, \hat{\mathbf{k}}) e^{i\left(-\Delta \mathbf{r}_{a} \cdot \mathbf{k}_{a}+\Delta \mathbf{r}_{b} \cdot\left(\hat{\mathbf{k}}+\mathbf{k}_{a}\right)\right)} \\
= & \left.\frac{1}{(2 \pi)^{4}} \iint P^{i\left(\Delta \mathbf{k}_{a} \cdot \mathbf{r}_{a}-\Delta \mathbf{k}_{b} \cdot\left(\hat{\mathbf{r}}+\mathbf{r}_{a}+L \mathbf{k}_{a} / k_{0}\right)\right)} d^{2} \hat{\mathbf{k}} d^{2} \hat{\mathbf{r}}\right) d^{2} \mathbf{k}_{a} d^{2} \mathbf{r}_{a} \\
& \int e^{i\left(\Delta \mathbf{r}_{b} \cdot \hat{\mathbf{k}}-\Delta \mathbf{k}_{b} \cdot \hat{\mathbf{r}}\right)} d^{2} \hat{\mathbf{k}} d^{2} \hat{\mathbf{r}} \\
&
\end{aligned}
$$

The integrals over $\mathbf{k}_{a}$ and $\mathbf{r}_{a}$ reduce to the product $(2 \pi)^{4} \boldsymbol{\delta}\left(\Delta \mathbf{r}_{b}-\Delta \mathbf{r}_{a}-\Delta \mathbf{k}_{b} L / k_{0}\right) \boldsymbol{\delta}\left(\Delta \mathbf{k}_{a}-\right.$ $\left.\Delta \mathbf{k}_{b}\right)$, or, equivalently $(2 \pi)^{4} \delta\left(\Delta \mathbf{r}_{b}-\Delta \mathbf{r}_{a}-\Delta \mathbf{k}_{a} L / k_{0}\right) \delta\left(\Delta \mathbf{k}_{a}-\Delta \mathbf{k}_{b}\right)$. We can now write the original four dimensional correlation function as,

$$
C\left(\Delta \mathbf{r}_{b}, \Delta \mathbf{k}_{b} ; \Delta \mathbf{r}_{a}, \Delta \mathbf{k}_{a}\right)=(2 \pi)^{2} \delta\left(\Delta \mathbf{r}_{b}-\Delta \mathbf{r}_{a}-\Delta \mathbf{k}_{a} L / k_{0}\right) \delta\left(\Delta \mathbf{k}_{b}-\Delta \mathbf{k}_{a}\right) C^{F P}\left(\Delta \mathbf{r}_{b}, \Delta \mathbf{k}_{b}\right),
$$

with

$$
C^{F P}\left(\Delta \mathbf{r}_{b}, \Delta \mathbf{k}_{b}\right) \equiv \frac{1}{(2 \pi)^{2}} \iint P^{F P}(\hat{\mathbf{r}}, \hat{\mathbf{k}}) e^{i\left(\Delta \mathbf{r}_{b} \cdot \hat{\mathbf{k}}-\Delta \mathbf{k}_{b} \cdot \hat{\mathbf{r}}\right)} d^{2} \hat{\mathbf{k}} d^{2} \hat{\mathbf{r}}
$$

Here, $C^{F P}$ is a generalized correlation function that is the forward and inverse 2D Fourier transforms along $\hat{\mathbf{r}}$ and $\hat{\mathbf{k}}$ of the associated light field transmission function, $P^{F P}(\hat{\mathbf{r}}, \hat{\mathbf{k}})$, for the shift and tilt invariant medium. We use $\langle P\rangle=P^{F P}$ for the particular case of comparing our results to the Fokker-Planck model (in Eq. (3.10)). However, Eq. (3.37) holds for any shift/tilt invariant light field transmission function. In the case of the Fokker-Planck model, the total transmission is unity, and the correlation coefficient in Eq. (3.37) is normalized. Otherwise, we may choose to normalize the correlation coefficient by dividing by $\frac{1}{(2 \pi)^{2}} \iint P(\hat{\mathbf{r}}, \hat{\mathbf{k}}) d^{2} \hat{\mathbf{k}} d^{2} \hat{\mathbf{r}}$, which is the total transmissivity of the sample.

\subsubsection{Correlation measurement}

In this section, we present additional mathematical details about our second experiment, where we directly measured the generalized correlation function. We performed our correlation measurements by first measuring many scattered field responses at the target plane for an input speckle field that we both shift and tilt via translation stages. Here, the sample is physically shifted by a distance $\Delta \mathbf{r}$ and the incident wavefront is tilted by $\Delta \mathbf{k}$ by translating the diffuser in front of the microscope objective. Hence, a single shifted/tilted measurement will take the form,

$$
E_{\Delta \mathbf{r}, \Delta \mathbf{k}}^{e x}\left(\mathbf{r}_{b}\right)=\int T\left(\mathbf{r}_{b}-\Delta \mathbf{r}, \mathbf{r}_{a}-\Delta \mathbf{r}\right) E_{a}\left(\mathbf{r}_{a}\right) e^{i \Delta \mathbf{k} \cdot \mathbf{r}_{a}} d^{2} \mathbf{r}_{a}
$$

We then perform a pairwise analysis of the measured fields. For each pair of fields, we have $\Delta \mathbf{r}_{a}=\Delta \mathbf{r}_{1}-\Delta \mathbf{r}_{2}$ and $\Delta \mathbf{k}_{a}=\Delta \mathbf{k}_{1}-\Delta \mathbf{k}_{2}$. For simplicity, and without loss of generality, we now only consider the symmetric case $\Delta \mathbf{r}_{1}=-\Delta \mathbf{r}_{2}=2 \Delta \mathbf{r}_{a}$ and $\Delta \mathbf{k}_{1}=-\Delta \mathbf{k}_{2}=2 \Delta \mathbf{k}_{a}$. 
After recording, we digitally shift and tilt the first field by $-\Delta \mathbf{r}_{b} / 2$ and $-\Delta \mathbf{k}_{b} / 2$, respectively, and we shift and tilt the second field by $\Delta \mathbf{r}_{b} / 2$ and $\Delta \mathbf{k}_{b} / 2$. Finally, we determine the overlap of the two fields, effectively calculating

$$
\begin{aligned}
C^{e x}\left(\Delta \mathbf{r}_{b}, \Delta \mathbf{k}_{b} ; \Delta \mathbf{r}_{a}, \Delta \mathbf{k}_{a}\right)=\int & {\left[\int T\left(\mathbf{r}_{b}^{+}, \mathbf{r}_{a^{\prime}}+\Delta \mathbf{r}_{a} / 2\right) E_{a}\left(\mathbf{r}_{a^{\prime}}\right) e^{i \Delta \mathbf{k}_{a} \cdot \mathbf{r}_{a^{\prime}} / 2} d^{2} \mathbf{r}_{a^{\prime}}\right] } \\
& {\left[\int T\left(\mathbf{r}_{b}^{-}, \mathbf{r}_{a^{\prime \prime}}-\Delta \mathbf{r}_{a} / 2\right) E_{a}\left(\mathbf{r}_{a^{\prime \prime}}\right) e^{-i \Delta \mathbf{k}_{a} \cdot \mathbf{r}_{a^{\prime \prime}} / 2} d^{2} \mathbf{r}_{a^{\prime \prime}}\right]^{*} e^{-i \Delta \mathbf{k}_{b} \cdot \mathbf{r}_{b}} d^{2} \mathbf{r}_{b} }
\end{aligned}
$$

with $\mathbf{r}_{b}^{ \pm} \equiv \mathbf{r}_{b} \pm \Delta \mathbf{r}_{b} / 2$. At this point, we wish to distinguish the effects of correlations in the transmission matrix and correlations in the incident field. For this purpose, we introduce the ambiguity function [13]

$$
A(\boldsymbol{\kappa}, \boldsymbol{\rho}) \equiv \int E_{a}\left(\mathbf{r}_{a}+\boldsymbol{\rho} / 2\right) E_{a}^{*}\left(\mathbf{r}_{a}-\boldsymbol{\rho} / 2\right) e^{-i \boldsymbol{\kappa} \cdot \mathbf{r}_{a}} d^{2} \mathbf{r}_{a}
$$

The ambiguity function describes the spatio-angular correlations in the input field. $A$ is linked to the Wigner distribution function of input field through Fourier transforms across $\boldsymbol{\kappa}$ and $\boldsymbol{\rho}$. By applying an inverse Fourier transform, we find

$$
E_{a}\left(\mathbf{r}_{a}+\boldsymbol{\rho} / 2\right) E_{a}^{*}\left(\mathbf{r}_{a}-\boldsymbol{\rho} / 2\right)=\frac{1}{(2 \pi)^{2}} \int A(\boldsymbol{\kappa}, \boldsymbol{\rho}) e^{i \boldsymbol{\kappa} \cdot \mathbf{r}_{a}} d^{2} \boldsymbol{\kappa}
$$

Next, we identify $\mathbf{r}_{a^{\prime}}=\mathbf{r}_{a}+\boldsymbol{\rho} / 2$ and $\mathbf{r}_{a^{\prime \prime}}=\mathbf{r}_{a}-\boldsymbol{\rho} / 2$ so that we can substitute this result into Eq. (3.39)

$$
\begin{gathered}
C^{e x}\left(\Delta \mathbf{r}_{b}, \Delta \mathbf{k}_{b} ; \Delta \mathbf{r}_{a}, \Delta \mathbf{k}_{a}\right)=\frac{1}{(2 \pi)^{2}} \iiint \int T\left(\mathbf{r}_{b}^{+}, \mathbf{r}_{a}+\boldsymbol{\rho} / 2+\Delta \mathbf{r}_{a} / 2\right) T^{*}\left(\mathbf{r}_{b}^{-}, \mathbf{r}_{a}-\boldsymbol{\rho} / 2-\Delta \mathbf{r}_{a} / 2\right) \\
e^{i\left(\Delta \mathbf{k}_{a} \cdot \mathbf{r}_{a}-\Delta \mathbf{k}_{b} \cdot \mathbf{r}_{b}+\boldsymbol{\kappa} \cdot \mathbf{r}_{a}\right)} A(\boldsymbol{\kappa}, \boldsymbol{\rho}) d^{2} \mathbf{r}_{b} d^{2} \mathbf{r}_{a} d^{2} \boldsymbol{\kappa} d^{2} \boldsymbol{\rho} .
\end{gathered}
$$

We can now substitute the definition of $C$ in Eq. (3.2) to arrive at

$$
C^{e x}\left(\Delta \mathbf{r}_{b}, \Delta \mathbf{k}_{b} ; \Delta \mathbf{r}_{a}, \Delta \mathbf{k}_{a}\right)=\frac{1}{(2 \pi)^{2}} \iint C\left(\Delta \mathbf{r}_{b}, \Delta \mathbf{k}_{b} ; \Delta \mathbf{r}_{a}+\boldsymbol{\rho}, \Delta \mathbf{k}_{a}+\boldsymbol{\kappa}\right) A(\boldsymbol{\kappa}, \boldsymbol{\rho}) d^{2} \boldsymbol{\kappa} d^{2} \boldsymbol{\rho}
$$

In words, the measured correlation function is the actual correlation function of the sample, convolved with the ambiguity function of the incident field mirrored along $\boldsymbol{\kappa}$ and $\boldsymbol{\rho}$.

Let us evaluate Eq. (3.43) for a correlation function for a shift/tilt invariant medium, as we found with the Fokker-Planck model. Substituting Eq. (3.37) into Eq. (3.43) gives

$$
\begin{aligned}
C^{e x}\left(\Delta \mathbf{r}_{b}, \Delta \mathbf{k}_{b} ; \Delta \mathbf{r}_{a}, \Delta \mathbf{k}_{a}\right)= & \iint C^{F P}\left(\Delta \mathbf{r}_{b}, \Delta \mathbf{k}_{b}\right) \delta\left(\Delta \mathbf{r}_{b}-\Delta \mathbf{r}_{a}-\boldsymbol{\rho}-\left(\Delta \mathbf{k}_{a}+\boldsymbol{\kappa}\right) L / k_{0}\right) \\
& \delta\left(\Delta \mathbf{k}_{b}-\Delta \mathbf{k}_{a}-\boldsymbol{\kappa}\right) A(\boldsymbol{\kappa}, \boldsymbol{\rho}) d^{2} \boldsymbol{\rho} d^{2} \boldsymbol{\kappa} \\
= & C^{F P}\left(\Delta \mathbf{r}_{b}, \Delta \mathbf{k}_{b}\right) A\left(\Delta \mathbf{k}_{b}-\Delta \mathbf{k}_{a}, \Delta \mathbf{r}_{b}-\Delta \mathbf{r}_{a}-\Delta \mathbf{k}_{b} L / k_{0}\right) .
\end{aligned}
$$


Let us now consider some typical experimental scenarios. First of all, for any incident beam with unit power, the ambiguity function will have a maximum at $A(0,0)=1$. If the incident beam approximates a plane wave, then $A$ will be sharp in the direction of $\boldsymbol{\kappa}$, and almost constant in the direction of $\boldsymbol{\rho}$. As a result, Eq. (3.44) predicts that we can vary $\Delta \mathbf{r}_{b}$ without observing an effect on the correlation. This result is to be expected since the incident beam is basically translational invariant, shifting it has no effect. This symmetry does not hold for the scattered wave, and thus $C^{e x}$ decreases with $\Delta \mathbf{r}_{b}$ in the same way as $C^{F P}$ decreases with $\Delta \mathbf{r}_{b}$. Finally, the fact that $A$ is sharp in the direction of $\kappa$ makes that tilt/tilt correlations are only observed when $\Delta \mathbf{k}_{b} \approx \Delta \mathbf{k}_{a}$, as is well-known from experimental observations of the tilt/tilt optical memory effect [6].

Second, if we focus the incident light onto the sample with a high numerical aperture, $A$ will be sharp in the direction of $\boldsymbol{\rho}$, and almost constant in the direction of $\boldsymbol{\kappa}$. In this case, the incident beam is tilt invariant, so we expect $C^{e x}$ not to remain constant when the incident beam is tilted (within the numerical aperture).

To summarize, we found that the angular correlations for an incident plane wave are only determined by the sample, whereas for a finite-size beam the correlations in the incident beam give rise to observed correlations over a larger angular range. This result is in line with observations by Li and Genack [7].

By creating a highly randomized input field for our correlation measurements, we were able to create a field with an ambiguity function that is sharp both along $\boldsymbol{\kappa}$ and $\boldsymbol{\rho}$, thus minimizing the impact of $A$ in this convolution and obtaining an accurate measurement of $C$. Additionally, in our experiment, we normalize the measured correlation coefficient and take the average over all pairs of measurement with the same spacing $\Delta \mathbf{r}_{a}$ and $\Delta \mathbf{k}_{a}$ to average over disorder.

\section{Acknowledgments}

This work was supported by the European Research Council (ERC-2016-StG-678919 and ERC-2016-StG-714560), NWO Vidi grant 14879, Einstein Foundation Berlin; DFG (EXC 257 NeuroCure), Human Frontier Science Program (RGP0027/2016) and the Krupp Foundation.

\section{Bibliography}

[1] J. Bertolotti, E. G. van Putten, C. Blum, A. Lagendijk, W. L. Vos, and A. P. Mosk, "Noninvasive imaging through opaque scattering layers", Nature 491, 232-234 (2012).

[2] O. Katz, E. Small, and Y. Silberberg, "Looking around corners and through thin turbid layers in real time with scattered incoherent light", Nat. Photonics 6, 549-553 (2012).

[3] O. Katz, P. Heidmann, M. Fink, and S. Gigan, "Non-invasive single-shot imaging through scattering layers and around corners via speckle correlations", Nat. Photonics 8, 784-790 (2014).

[4] X. Yang, Y. Pu, and D. Psaltis, "Imaging blood cells through scattering biological tissue using speckle scanning microscopy”, Opt. Express 22, 3405-3413 (2014). 
[5] I. Freund, M. Rosenbluh, and S. Feng, "Memory effects in propagation of optical waves through disordered media”, Phys. Rev. Lett. 61, 2328-2331 (1988).

[6] S. Feng, C. Kane, P. A. Lee, and A. D. Stone, "Correlations and fluctuations of coherent wave transmission through disordered media”, Phys. Rev. Lett. 61, 834-837 (1988).

[7] J. Li and A. Genack, “Correlation in laser speckle”, Phys. Rev. E 49, 4530-4533 (1994).

[8] S. Schott, J. Bertolotti, J.-F. Léger, L. Bourdieu, and S. Gigan, "Characterization of the angular memory effect of scattered light in biological tissues", Opt. Express 23, 13505-13516 (2015).

[9] B. Judkewitz, R. Horstmeyer, I. M. Vellekoop, I. N. Papadopoulos, and C. Yang, “Translation correlations in anisotropically scattering media", Nat. Phys. 11, 684-689 (2015).

[10] J. Mertz, H. Paudel, and T. G. Bifano, "Field of view advantage of conjugate adaptive optics in microscopy applications", Appl. Opt. 54, 3498-3506 (2015).

[11] M. J. Bastiaans, "Wigner distribution function and its application to first-order optics", J. Opt. Soc. Am. 69, 1710-1716 (1979).

[12] M. Testorf, B. Hennelly, and J. Ojeda-Castaneda, Phase-Space Optics: Fundamentals and Applications (McGraw-Hill Education) (2009).

[13] Z. Zhang and M. Levoy, "Wigner distributions and how they relate to the light field", in Computational Photography (ICCP), 2009 IEEE International Conference on, 1-10 (IEEE) (2009).

[14] H.-Y. Liu, E. Jonas, L. Tian, J. Zhong, B. Recht, and L. Waller, “3d imaging in volumetric scattering media using phase-space measurements”, Opt. Express 23, 14461-14471 (2015).

[15] J.-H. Park, W. Sun, and M. Cui, "High-resolution in vivo imaging of mouse brain through the intact skull”, Proc. Natl. Acad. Sci. U.S.A. 12, 9236-9241 (2015).

[16] A. Wax and E. Thomas, "Measurement of smoothed wigner phase-space distributions for small-angle scattering in a turbid medium", J. Opt. Soc. Am. A 15, 1896-1908 (1998).

[17] C.-C. Cheng and M. Raymer, "Propagation of transverse optical coherence in random multiple-scattering media", Phys. Rev. A 62, 023811 (2000).

[18] I. Papadopoulos, J. Jouhanneau, J. Poulet, and B. Judkewitz, "Scattering compensation by focus scanning holographic aberration probing (F-SHARP)", Nat. Photonics 11, 116-123 (2017).

[19] C. W. Hsu, S. F. Liew, A. Goetschy, H. Cao, and A. D. Stone, "Correlation-enhanced control of wave focusing in disordered media", Nat. Phys. 13, 497-502 (2017).

[20] S. Chandrasekhar, "Stochastic problems in physics and astronomy", Rev. Mod. Phys. 15, 1-89 (1943). 
[21] H. Risken, The Fokker-Planck Equation. Methods of Solution and Applications (Springer Series in Synergetics) (1989).

[22] I. A. Tanski, "Fundamental solution of fokker-planck equation", arXiv: nlin/0407007 (2004). 


\section{4}

\section{Shaping light to enhance the imaging performance of two-photon microscopy}

In this chapter, we bring together concepts learned from the previous two chapters to enhance the imaging performance of a two-photon excitation microscope (TPM). First we will go over the basic principles of two-photon microscopy. Afterwards, we present the implementation of a wavefront shaping algorithm in our custom-built TPM setup, using the detected fluorescence signal as a source of feedback. Finally, we introduce a new imaging scheme called digital conjugate adaptive optics (DCAO), which is derived from the generalized optical memory effect. With DCAO, the wavefront correction can be digitally projected to any plane in the sample. Using this method, we will demonstrate an 8-fold increase of the isoplanatic patch size through a scattering layer.

\subsection{Two-photon excitation microscopy}

Modern fluorescence microscopes allow the observation of specific types of tissue and intracellular structures, sometimes up to single molecule resolutions [1]. However, due to sample induced scattering and out-of-focus light, standard microscopes require samples to be sliced up into thin layers of only 10 to $20 \mu \mathrm{m}$ [2]. To overcome these sample restrictions, Denk et al. introduced two-photon excitation microscopy (TPM) [3]. In TPM, an image is formed by collecting the emitted fluorescent light using a detector while scanning a focus over the fluorescent structures inside a sample. The basic principles of TPM are illustrated in Fig. 4.1.

As the name describes, TPM makes use of two-photon excitation which was first predicted by Maria Göppert-Mayer [4] and later first observed by Kaiser et al. [5] after the invention of the laser. In two-photon excitation, the energy of two lower-energy (longer wavelength) photons is combined to excite the fluorescent markers, rather than the energy of 

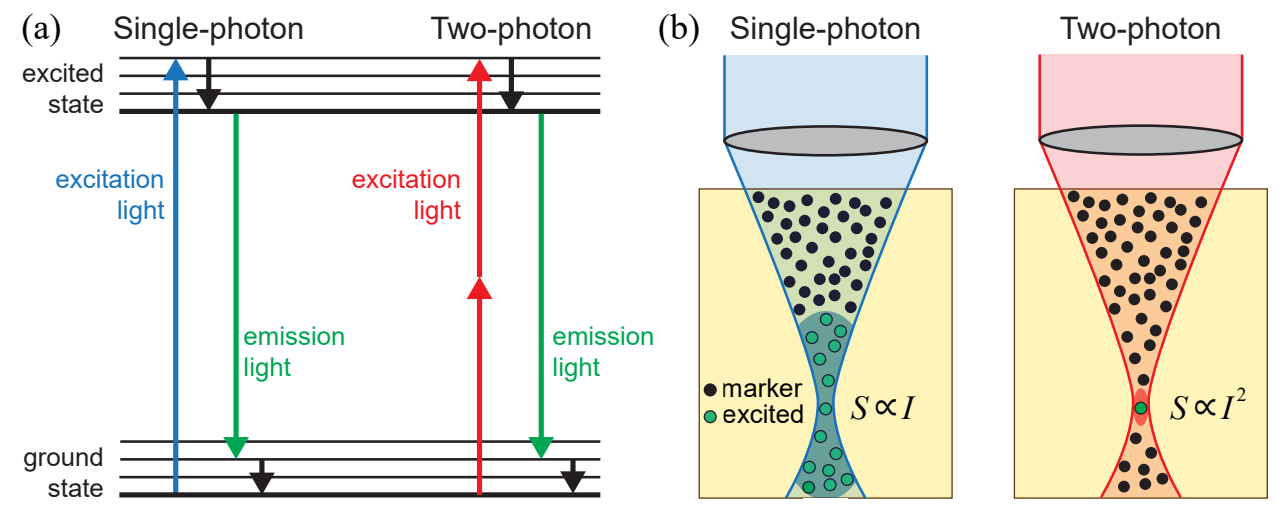

Figure 4.1: Basic of principles of two-photon excitation microscopy. (a) A Jablonski diagram illustrating the electronic states of a fluorescent molecule. The colored arrows represent the transitions between the ground state and the excited state, as a result of light absorption or emission. The black arrows represent non-radiant internal energy conversion. (b) Excitation of fluorescent markers inside a three-dimensional sample. A large volume with markers is excited with single-photon excitation, as the generated signal $S$ is proportional to the laser intensity $I$. In the two-photon excitation, $S \propto I^{2}$, and therefore only the fluorescent markers at the focus location are excited.

a single photon (see Fig. 4.1a). Therefore, in this second-order process, the amount of emitted light is proportional to the squared intensity of the excitation light. As shown in Fig. 4.1b, the excitation beam will mostly excite the fluorescent markers at the focus location with twophoton excitation, whereas with single-photon excitation markers in a much larger volume are excited. This localized excitation volume is used as an optical sectioning mechanism to image inside intact tissue volumes [6]. Two-photon microscopy revolutionized biological research by enabling high-resolution imaging of thicker unperturbed environments [2].

However, the signal generated by two-photon excitation is many orders of magnitude weaker than single-photon excitation [7]. For this reason, TPM requires a sharp and intense excitation focus. The amount of ballistic light, forming this focus, decreases exponentially with increasing imaging depth. As a result, the maximum achievable imaging depth of a TPM is typically limited to 3 scattering mean free path lengths [8]. This depth limit can be extended by using high peak power lasers [9], or by using longer excitation wavelengths [10]. However, these solutions still fail to acquire images at depths where light no longer forms a focus.

In the next section, we will explore how the fundamental depth limit of two-photon excitation microscopy can be broken by means of feedback-based wavefront shaping [11]. The potential of this technique is tested in our custom-built TPM setup (see Appendix 4.5.1). In this setup, we included a phase-only spatial light modulator (SLM), allowing us to shape the wavefront of the excitation light. The SLM can correct for the light scattering, which enhances the focus intensity and enables TPM images to be acquired at larger depths inside the sample. 


\subsection{Focus optimization using fluorescence feedback}

\subsubsection{Localized source of feedback}

Feedback-based wavefront shaping relies on the detection of the light intensity at a location of interest. This intensity signal can be provided by a small probe inside the scattering sample, commonly referred to as a guide star [12]. In TPM, the feedback signal can be acquired from the fluorescent structures embedded in the sample. When attempting to focus in biological tissue, the light intensity will be mostly forward scattered, and will end up in a small volume around the intended focus location. From Chapter 2 we learned that only the strongly illuminated parts of the fluorescent structure will contribute to the feedback signal. Assuming the sample is sparsely labeled and the scattered light is sufficiently localized, we can select a single guide star which is used for feedback. Therefore, the laser beam is parked on the guide star of interest prior to the optimization procedure.

\subsubsection{Two-step optimization algorithm}

Now that a localized form of feedback is established, we form a focus by maximizing the amount of fluorescent light emitted by the guide star. For the focus optimization, we use a two-step optimization algorithm introduced by Tao et al. [13]. In the two-step algorithm, the SLM is subdivided into two equally sized groups of pixels. During the first measurement step, the first group acts as the active group, while the second group is used as a static reference. The active group is used to construct a set of $N / 2$ orthogonal Hadamard patterns. For each pattern, the feedback signal is measured as the phase of the active group is varied from 0 to $2 \pi$ in $P$ steps. From these phase stepping measurements, we can obtain the amplitude and phase of the input mode with respect to the reference field (see Chapter 2 for details of the analysis). Then, the ideal wavefront for group 1 is found by taking the superposition of the Hadamard modes with their corresponding complex amplitudes. In step 2, the same procedure is repeated with group 2 as the active group, and the optimized wavefront on group 1 as the reference field. Similarly to the algorithm described by Yilmaz et al. [14], the partially optimized focus from group 1 acts as a pre-optimization step here. As a result, the second step in the optimization procedure benefits from an increased signal-to-noise ratio. Finally, after NP measurements, the optimal wavefront for the full incident field is found and a single sharp focus is formed at the desired location.

\subsubsection{Focusing experiment}

The two-step optimization algorithm is tested using our custom-built two-photon microscope, which is described in Appendix 4.5.1. In this setup, the wavefront of the light is shaped by an SLM, which is conjugated to the pupil-plane. The algorithm is tested on a polydimethylsiloxane (PDMS) diffuser sample. The sample preparation protocol is described in Appendix 4.5.2. The sample has a rough surface which scatters the light and prevents a sharp focus from being formed. Inside the PDMS sample we dispersed $0.5 \mu \mathrm{m}$ fluorescent beads, which act as guide stars for the feedback-based wavefront shaping algorithm. To prevent the guide star from drifting out of focus, we collect feedback by scanning the beam over a small area of $2 \times 2 \mu \mathrm{m}^{2}$ around the guide star. We then maximize the mean intensity within this area by performing the optimization algorithm with $N=256$ Hadamard patterns and $P=8$ 

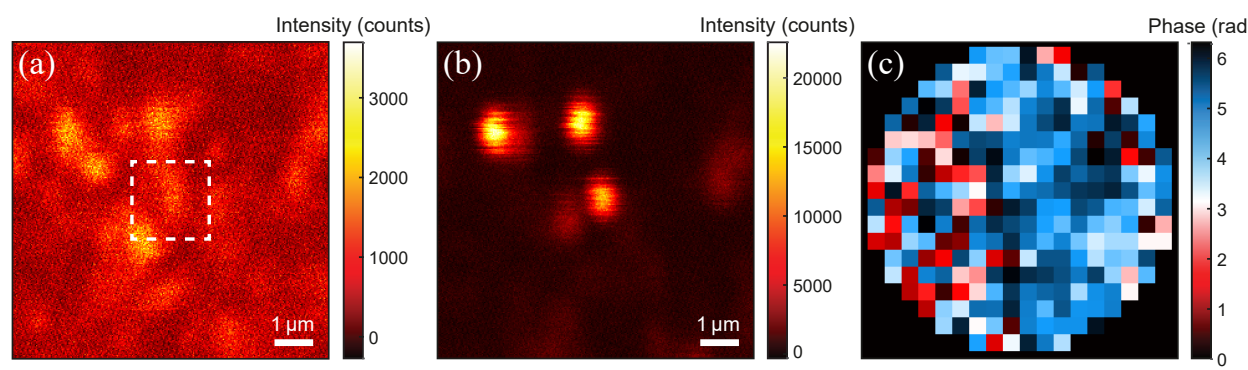

Figure 4.2: Wavefront shaping experiment at $150 \mu \mathrm{m}$ depth inside the PDMS diffuser sample. (a) TPM image without a wavefront correction. The dashed white square indicates the area used for feedback for the optimization algorithm. (b) TPM image with wavefront correction. (c) Wavefront correction obtained with the two-step optimization algorithm.

steps. The wavefront shaping experiments were performed at a depth of $150 \mu \mathrm{m}$ inside the PDMS sample

Fig. 4.2a and b show the TPM images acquired without and with the wavefront correction, respectively. The sample area used for feedback is indicated by the dashed white square in Fig. 4.2a. The corresponding phase pattern constructed with the SLM is presented in Fig. 4.2c. The uncorrected image suffers from a low contrast between the beads and the background intensity. After the optimization, the beads can be clearly discerned from the background, as the image intensity of the beads is enhanced by an average factor of 9.5.

\subsection{Enhanced imaging through a scattering layer}

Focusing light on a guide star inside a scattering sample is not the only requirement for imaging. The optimized focus also has to be scanned to probe the fluorescent structures surrounding the guide star. However, as the focus is scanned, the light will experience scattering of a different part of the sample. Therefore, the effective imaging field-of-view is limited to an area known as the isoplanatic patch. When imaging outside this area, the focus is lost and has to be re-optimized.

\subsubsection{Conjugate adaptive optics}

The size of the isoplanatic patch is governed by the optical memory effect [15-18] of the sample. However, the applied imaging scheme also strongly affects the isoplanatic patch size. This is particularly the case in samples where the scattering can mostly be attributed to a single layer, i.e. a sample with a rough surface. Using a technique known as conjugate adaptive optics [19], the SLM is projected onto the scattering layer, canceling the wavefront distortions introduced by that layer. The wavefront correction will remain static at the scattering layer as the focus is being laterally scanned to form an image. As a result, the isoplanatic patch through this scattering layer can be greatly extended [13, 19, 20]. For example, in an in vivo experiment, Park et al. [21] were able to image neurons through the intact skull of a mouse, by conjugating the SLM to the plane of the skull. 

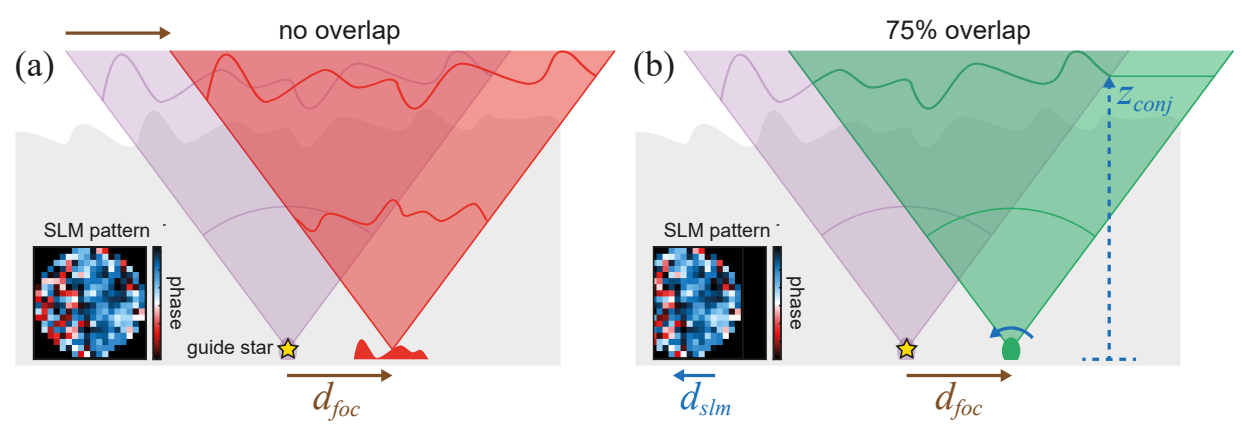

Figure 4.3: Concept of digital conjugate adaptive optics using a pupil-conjugated SLM. (a) Conventional telecentric focus scanning, shifting the wavefront correction at the sample surface and resulting in a distorted focus. (b) Digital conjugate adaptive optics imaging scheme, where the SLM pattern is shifted in the direction opposite to the focus scan direction. As a result, the wavefront correction remains stationary at the sample surface and a sharp focus is maintained after scanning. The insets show the phase patterns displayed on the SLM.

The implementation of conjugate adaptive optics, however, greatly increases the complexity of the microscope setup. The SLM has to be physically moved to the correct conjugation plane while leaving the setup alignment intact. Therefore, this added complexity generally leads to additional system aberrations affecting the imaging performance of the microscope. Moreover, finding the optimal conjugation plane might be a time-consuming process as the exact depth of the strongest scattering layer might not always be known. In the next section, we will introduce a new method that allows us to benefit from the extended isoplanatic patch from conjugate adaptive optics while using a simple TPM setup with a static SLM conjugated to the pupil-plane of the microscope objective lens.

\subsubsection{Digital conjugate adaptive optics}

In this section, we will introduce a method called digital conjugate adaptive optics (DCAO) based on the generalized optical memory effect [18]. This new imaging scheme allows us to digitally project our pupil-conjugated SLM to any plane within the sample. The basic concept of DCAO is illustrated in Fig. 4.3.

After optimizing the focus with an SLM, the optimized focus is conventionally scanned using scanning mirrors. In this telecentric scanning scheme the wavefront correction is translated with respect to the sample. As shown in Fig. 4.3a, the scanned focus will be distorted due to the mismatch between the wavefront correction and the scattering layer. In Fig. 4.3b, we employ DCAO, where the SLM pattern is shifted as the focus is scanned. In the paraxial limit, a translation of the pupil-conjugated SLM of $d_{s l m}$ leads to a tilt of $d_{s l m} / f_{o b j}$ radians at the focal plane. Here, $f_{o b j}$ is the effective focal length of the objective lens. This tilt results in a lateral displacement of $\left(d_{s l m} / f_{o b j}\right) z$ at a plane at a distance $z$ from the focal plane. By simultaneously scanning the focus over a distance $d_{f o c}$, while shifting the SLM in the opposite direction, the wavefront correction will remain stationary at a single plane inside the sample. 

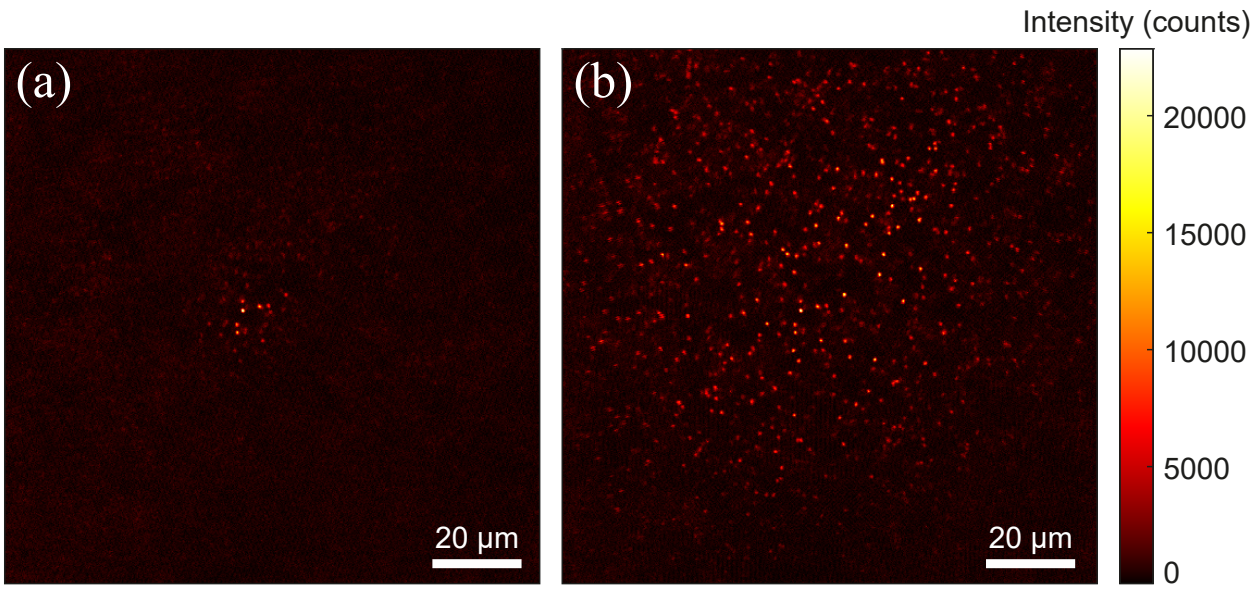

Figure 4.4: DCAO imaging experiment. (a) Image acquired by scanning the focus with the SLM conjugated to the pupil plane. (b) DCAO image (combination of 121 sub-images) with an extended isoplanatic patch, obtained by digitally conjugating the SLM to the surface of the PDMS sample. The same optimized wavefront was used for both images.

Given that the focal plane is at $\mathrm{z}=0$, this conjugation plane will be located at

$$
z_{c o n j}=\frac{d_{f o c}}{d_{s l m} / f_{o b j}} .
$$

In DCAO, an image is formed by combining multiple sub-images. We first scan focus using the scanning mirrors to capture a sub-image of the close surroundings of the guide star. Afterwards, the center of the isoplanatic patch is moved by shifting the pattern on the pupilconjugated SLM. The following sub-image is then captured at the shifted isoplanatic patch, where a different part of the image is enhanced. This process is repeated until finally all the sub-images are stitched together to form the full DCAO image.

DCAO allows us to scan the focus over a larger area without the need to repeat the wavefront shaping algorithm. Here, the acquisition speed is limited to the number of sub-images and the refresh rate of the SLM. This method is in principle equivalent to conjugate adaptive optics, yet it can be implemented without physically moving the SLM to the correct conjugation plane. The application of DCAO is not limited to single scattering layers only. In Chapter 3, we found that an optimal conjugation also exists in volumetric scattering media. Conjugating the SLM to this optimal plane should in that case double the size of the isoplanatic patch.

\subsubsection{Proof-of-principle imaging experiment}

In a proof-of-principle imaging experiment, we will demonstrate an improvement of isoplanatic patch size using DCAO. As a scattering, we use the PDMS diffuser discussed in the previous section. We use the same wavefront correction obtained during the wavefront shaping experiments (see Fig. 4.2c). Using this correction, we now acquire a TPM image with a total field-of-view $132 \times 132 \mu \mathrm{m}^{2}$, at a depth of $150 \mu \mathrm{m}$. 
Fig. 4.4a shows the TPM image acquired by conventional focus scanning using scanning mirrors. Here, the pattern on the pupil-conjugated SLM is not altered during the image acquisition. In this image, the fluorescent beads close to the center of the image (the guide star location) again show an enhancement of a factor of 9.5. However, outside this area, the beads can no longer be observed, as the wavefront correction no longer cancels the distortions introduced by the sample surface. The isoplanatic patch size is estimated to be $12 \mu \mathrm{m}$ wide.

Using the same wavefront correction, we acquired a DCAO image by stitching together 121 sub-images. In between the acquisition of each sub-image, we shift the SLM pattern over a distance of $0.92 \mathrm{~mm}$. As a result, the center of the isoplanatic patch is displaced by $12 \mu \mathrm{m}$, correcting for a different part of the image plane. Finally, by combining the 121 sub-images, we obtain the composite DCAO image, which is presented in Fig. 4.4b. In this image, the fluorescent beads can be observed at a larger distance from the image center than in Fig. 4.4a. In this DCAO image, the isoplanatic patch was estimated to be $95 \mu \mathrm{m}$ wide. Here, the size of this area is proportional to the size of the illumination spot on the sample surface, which is also the limit for conventional conjugate adaptive optics [19].

\subsection{Summary}

In this chapter, we first described the basic principles of two-photon excitation microscopy. Then, using our custom-built TPM setup, we demonstrated enhanced imaging through a scattering layer using a feedback-based wavefront shaping algorithm. We were able to improve the intensity of the fluorescent beads inside a PDMS test sample by a factor of 9.5. However, the full optimization procedure took roughly 5 minutes to be completed. Therefore, we introduced a new method we call digital conjugate adaptive optics to extend the isoplanatic patch without the need to reoptimize the focus. This method allows us to benefit from the advantages of conjugate adaptive optics [19] without physically moving the SLM. Using DCAO, we extended the size of the isoplanatic patch by a factor of 8 at a depth of $150 \mu \mathrm{m}$ inside a PDMS sample with a rough surface.

\subsection{Appendices}

\subsubsection{Microscope setup}

The two-photon fluorescence excitation microscopy (TPM) setup is illustrated in Fig. 4.5. A titanium-sapphire laser (Spectra-Physics, Mai Tai) is used as the light source for two-photon excitation at a wavelength of $804 \mathrm{~nm}$. The power and the polarization of the laser beam are controlled using a half-wave plate (HWP) and a polarizing beam splitter (PBS). After a $10 \times$ beam expander, the laser light is sent to two galvo mirrors (GM1 and GM2) which are used to vertically and horizontally scan the beam. A spatial light modulator (SLM, LC, Meadowlark Optics, $1920 \times 1152$ pixels) is conjugated to the pupil plane of the objective lens (Nikon, CFI75 LWD 16x, numerical aperture of 0.8), which focuses the laser light onto the sample. The sample is placed on a 3D stage to facilitate initial alignment. A piezo scanning stage (PI, PD72Z2x/4x) allows the objective lens to be moved for depth scanning. To separate the emitted fluorescent light from the laser light, a dichroic mirror (DM, Semrock, FF685-Di02-25×36) and a short pass transmission filter (SPF, Semrock, FF01-680/SP-25) 


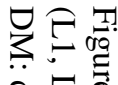

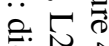

实

는

ㅋ. 음

5

的冚

T)

它

平

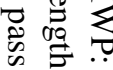

를

鱼实

38 춘

ज. 80

$\exists$.

둥

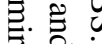

훙

क 8

送

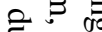

言.

ㅋ.․ 을

.

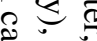

可

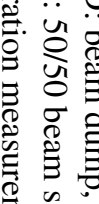

急

莺

응

3

年

苛.

추

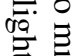

쿵

$\stackrel{5}{\circ}$

产

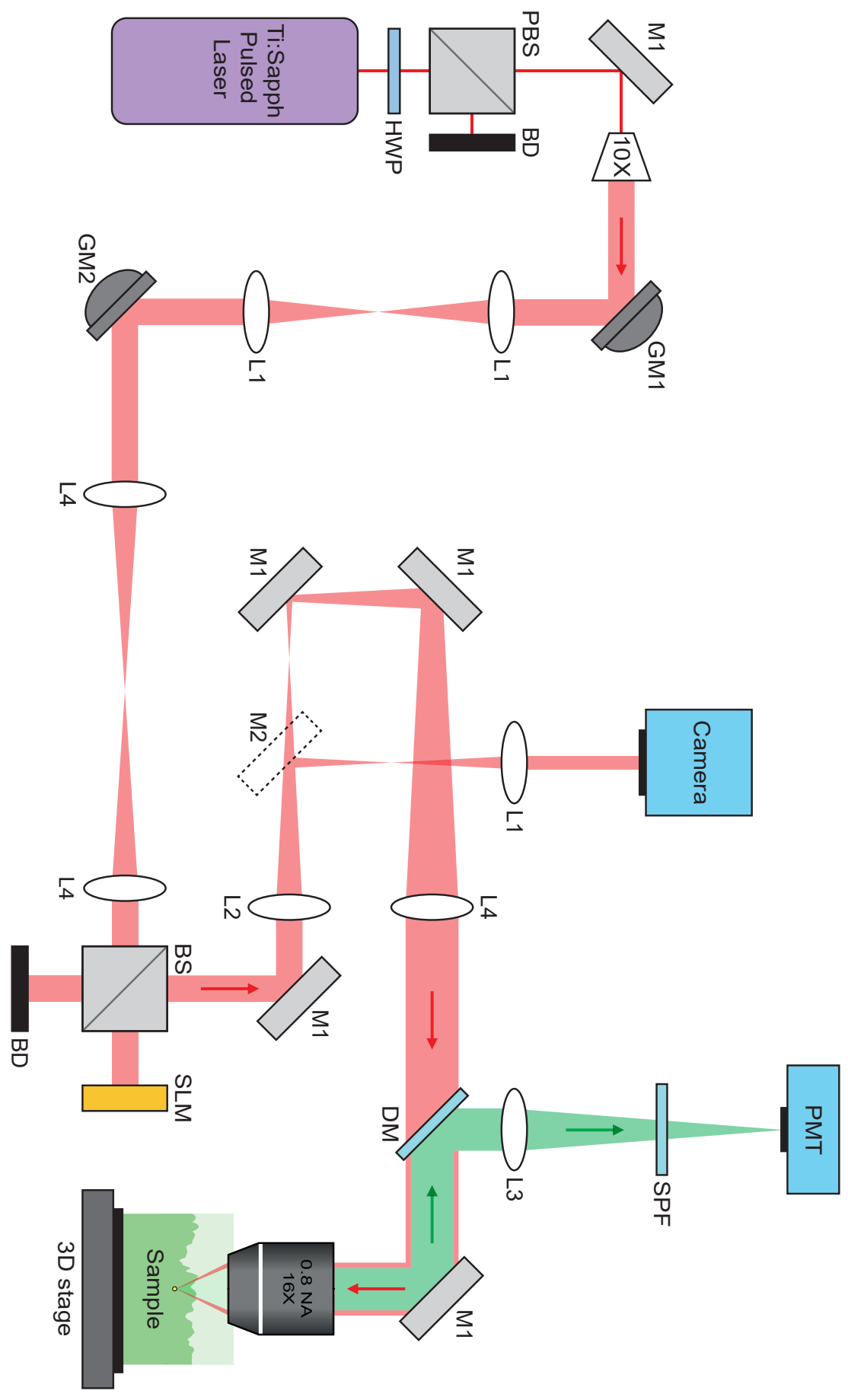




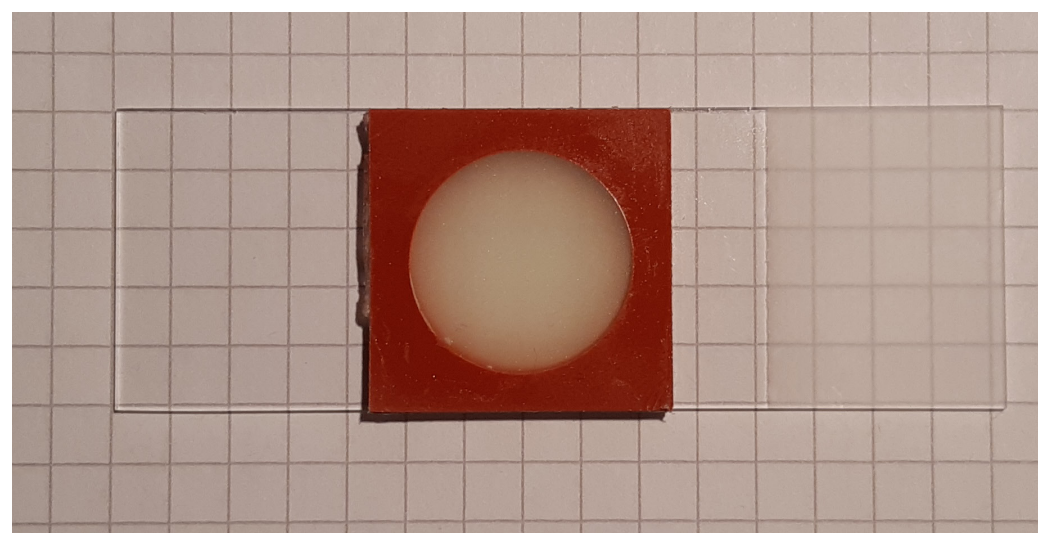

Figure 4.6: PDMS diffuser dispersed with $500 \mathrm{~nm}$ fluorescent beads.

are used. Finally, the fluorescent light is detected by a photomultiplier tube (PMT, Hamamatsu, H10770(P)A-40/-50). Mirror M2 is mounted on a flip stage and can be flipped to redirect the laser light to a camera (Basler, acA2000-165umNIR). This camera is used to image the light distribution on the SLM for initial calibration measurements. The TPM images are acquired using ScanImage [22], an open-source software (https://scanimage.org).

\subsubsection{Sample preparation}

We used the following protocol to make a polydimethylsiloxane (PDMS) diffuser dispersed with fluorescent beads. First, we mixed the fluorescent beads (Fluoresbrite, plain YG, 500 $\mathrm{nm}$ microspheres) with a Triton X-100 + Water + Ethanol (1:1:1) solution in a 1:2 ratio to avoid cluster formation. The resulting solution was mixed with PDMS (DMS base + curing agent in 10:1 ratio, Sylgrad 184, Dow Silicones) in a 1:66 ratio. In order to disperse the microspheres uniformly, we grinded this mix for 20 minutes. Afterwards, we put the solution in a vacuum chamber and removed the air bubbles. The resulting solution was centrifuged at 2000 RPM for 5 minutes. The single diffusive layer of PDMS was formed by pouring the mix into a $1.5 \mathrm{~mm}$ silicon spacer placed on the surface of a ground glass diffuser (120 grit, custom-made). The PDMS was cured at $50{ }^{\circ} \mathrm{C}$ for 2 hours and finally glued to a microscope slide. Fig. 4.6 shows an image of the sample. A characterization of the scattering properties of the sample can be found in Chapter 5 .

\section{Bibliography}

[1] E. Betzig, G. H. Patterson, R. Sougrat, O. W. Lindwasser, J. S. Olenych, S. Bonifacino, M. W. Davidson, J. Lippincott-Schwartz, and H. F. Hess, "Imaging intracellular fluorescent proteins at nanometer resolution”, Science 313, 1642-1645 (2006).

[2] V. Ntziachristos, "Going deeper than microscopy: the optical imaging frontier in biology”, Nat. Methods 7, 603-614 (2010). 
[3] W. Denk, J. H. Strickler, and W. W. Webb, “Two-photon laser scanning fluorescence microscopy", Science 248, 73-76 (1990).

[4] M. Göppert-Mayer, “Über Elementarakte mit zwei Quantensprüngen”, Ann. Phys. (Berl.) 401, 273-294 (1931).

[5] W. Kaiser and C. G. B. Garrett, “Two-photon excitation in caf 2 : eu ${ }^{2+}$, Phys. Rev. Lett. 7, 229-231 (1961).

[6] F. Helmchen and W. Denk, "Deep tissue two-photon microscopy", Nat. Methods 2, 932-940 (2005).

[7] C. Xu, R. M. Williams, W. Zipfel, and W. W. Webb, "Multiphoton excitation crosssections of molecular fluorophores", Bioimaging 4, 198-207 (1996).

[8] P. Theer and W. Denk, "On the fundamental imaging-depth limit in two-photon microscopy”, J. Opt. Soc. Am. A 23, 3139-3149 (2006).

[9] R. Kawakami, K. Sawada, Y. Kusama, Y.-C. Fang, S. Kanazawa, Y. Kozawa, S. Sato, H. Yokoyama, and T. Nemoto, "In vivo two-photon imaging of mouse hippocampal neurons in dentate gyrus using a light source based on a high-peak power gain-switched laser diode”, Biomed. Opt. Express 6, 891 (2015).

[10] D. Kobat, M. E. Durst, N. Nishimura, A. W. Wong, S. C. B., and C. Xu, "Deep tissue multiphoton microscopy using longer wavelength excitation”, Opt. Express 17, 1335413364 (2009).

[11] I. M. Vellekoop, "Feedback-based wavefront shaping", Opt. Express 23, 12189-12206 (2015).

[12] R. Horstmeyer, H. R., and C. Yang, "Guidestar-assisted wavefront-shaping methods for focusing light into biological tissue”, Nat. Photonics 9, 563-571 (2015).

[13] X. Tao, T. Lam, B. Zhu, Q. Li, M. R. Reinig, and J. Kubby, "Three-dimensional focusing through scattering media using conjugate adaptive optics with remote focusing (CAORF)", Opt. Express 25, 10368 - 10383 (2017).

[14] H. Yilmaz, W. L. Vos, and A. P. Mosk, "Optimal control of light propagation through multiple-scattering media in the presence of noise", Biomed. Opt. Express 4, 17591768 (2013).

[15] S. Feng, C. Kane, P. A. Lee, and A. D. Stone, "Correlations and fluctuations of coherent wave transmission through disordered media", Phys. Rev. Lett. 61, 834-837 (1988).

[16] I. Freund, M. Rosenbluh, and S. Feng, "Memory effects in propagation of optical waves through disordered media”, Phys. Rev. Lett. 61, 2328-2331 (1988).

[17] B. Judkewitz, R. Horstmeyer, I. M. Vellekoop, I. N. Papadopoulos, and C. Yang, "Translation correlations in anisotropically scattering media", Nat. Phys. 11, 684-689 (2015).

[18] G. Osnabrugge, R. Horstmeyer, I. N. Papadopoulos, B. Judkewitz, and I. M. Vellekoop, "Generalized optical memory effect", Optica 4, 886-892 (2017). 
[19] J. Mertz, H. Paudel, and T. G. Bifano, "Field of view advantage of conjugate adaptive optics in microscopy applications”, Appl. Opt. 54, 3498-3506 (2015).

[20] H. P. Paudel, J. Taranto, J. Mertz, and T. Bifano, “Axial range of conjugate adaptive optics in two-photon microscopy", Opt. Express 23, 20849-20857 (2015).

[21] J.-H. Park, W. Sun, and M. Cui, "High-resolution in vivo imaging of mouse brain through the intact skull”, Proc. Natl. Acad. Sci. U.S.A. 112, 9236-9241 (2015).

[22] T. A. Pologruto, B. L. Sabatini, and K. Svoboda, "Scanimage: Flexible software for operating laser scanning microscopes”, Biomed. Eng. Online 2, 13 (2003). 


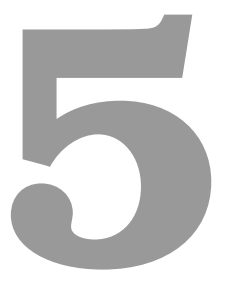

\section{Model-based wavefront shaping microscopy}

Wavefront shaping is increasingly being used in modern microscopy to obtain high-resolution images deep inside inhomogeneous media. Wavefront shaping methods typically rely on the presence of a 'guide star' to find the optimal wavefront to mitigate the scattering of light. However, the use of guide stars poses severe limitations. Notably, only objects in the close vicinity of the guide star can be imaged. Here, we introduce a guide-star-free wavefront shaping method in which the optimal wavefront is computed using a digital model of the sample. The refractive index model of the sample, that serves as the input for the computation, is constructed in-situ by the microscope itself. In a proof of principle imaging experiment, we demonstrate a large improvement in the two-photon fluorescence signal through a diffuse medium, outperforming state-of-the-art wavefront shaping by a factor of two in imaging depth.

\subsection{Introduction}

Imaging deep inside biological tissues at a high resolution is a long sought-after goal in microscopy. This task is extremely challenging as inhomogeneities in the refractive index scatter light, preventing the formation of a sharp focus. This problem can be overcome by shaping the wavefront of the incident light to counteract the scattering. Recent progress in wavefront shaping has enabled sub-wavelength resolution imaging through turbid media in several proof-of-concept experiments [1].

This chapter has been published as: [A. Thendiyammal, G. Osnabrugge, T. Knop and I.M. Vellekoop, arXiv:2002.05279 (2020)]. 


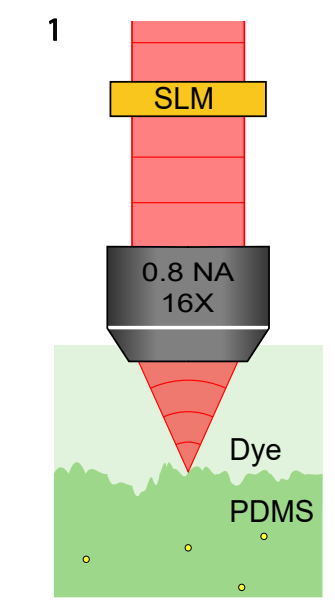

Refractive index mapping
2

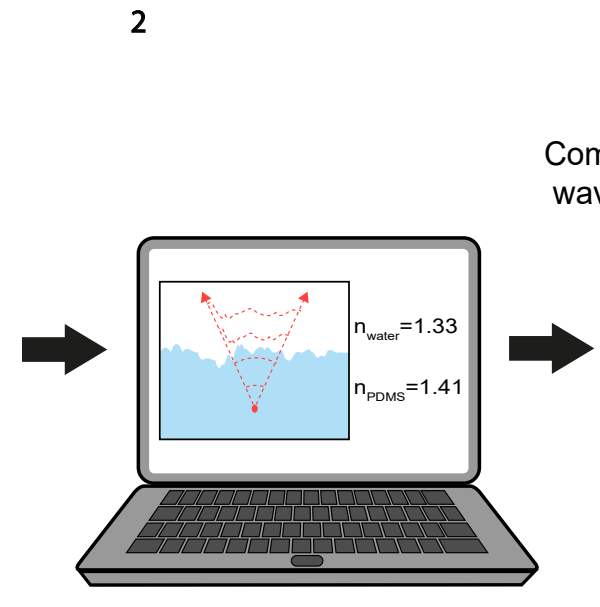

Modeling
3

3

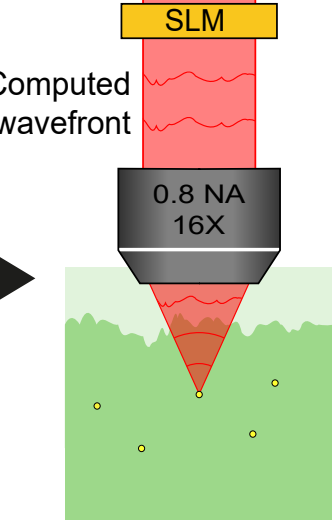

Phase conjugation

Figure 5.1: Principle of model-based wavefront shaping microscopy. Step 1: the superficial region of the sample is imaged and a $3 \mathrm{D}$ model of the refractive index is generated from the image data. Step 2: the model is used to compute the wavefront required to focus at the desired location. Step 3: the computed wavefront is constructed with a spatial light modulator (SLM), resulting in a sharp focus.

In wavefront shaping, two main classes of approaches can be distinguished: feedbackbased wavefront shaping [2] and optical phase conjugation [3]. Feedback-based methods depend on the detection of the intensity at a desired focus location, either directly [4] or indirectly through the use of a guide star [5]. By iteratively maximizing this feedback signal a sharp focus is formed. An alternative approach to focus light inside turbid media is optical phase conjugation. Here, the optimal wavefront is obtained by measuring the scattered field coming from a guide star inside the medium. Subsequently, a focus is formed by playing back the conjugate of this field using a phase conjugating mirror [5]. Many types of guide stars have been used, including second harmonic generation [6], fluorescence [7], and moving particles [8, 9].

The use of guide stars, however, poses severe limitations. First of all, it is not always feasible to embed guide stars, or to obtain a signal with a sufficient signal to noise ratio (SNR) to form a high-quality focus. Also, when multiple guide stars are too close together, special care needs to be taken to focus on exactly one of them [10-12]. Even more importantly, once a correction is found, it is only valid for a small region around the guide star [13], so only objects in its close vicinity can be imaged. A notable exception here is the use of ultrasound tagging to generate a virtual guide star at any desired location [14, 15]. Unfortunately, here the resolution is limited by the size of the ultrasound focus, which is of the order of tens of micrometers. To summarize, the problem of tightly focusing light at an arbitrary location inside a scattering medium is still unsolved.

Here, we introduce a third class of wavefront shaping methods, which we call modelbased wavefront shaping. In model-based wavefront shaping, the optimal wavefront is computed numerically using a digital model of the sample. The microscopic refractive index 
model of the sample, that serves as the input for the calculations, is obtained from the image data itself. With this method, no guide stars are required and the light can be focused anywhere; provided that an accurate refractive index model can be constructed.

The concept of model-based wavefront shaping is illustrated in Fig. 5.1. First, we generate a refractive index distribution model by imaging the superficial region of the scattering sample and applying a priori knowledge about the materials in the sample. Once the refractive index model is generated, it is possible to compute the wavefronts required to focus light anywhere inside the sample by performing a virtual (digital) phase conjugation experiment: we place a 'virtual guide star' in our model and simulate the propagation of light from that point to outside the sample. This calculated field is phase conjugated and then constructed experimentally with a spatial light modulator (SLM). As with ordinary phase conjugation, the conjugated field will propagate through the scattering sample and form a sharp focus.

\subsection{Proof of concept experiment}

As a proof of concept of our method, we demonstrate enhanced imaging of $500 \mathrm{~nm}$ fluorescent beads dispersed in polydimethylsiloxane (PDMS). The sample has a rough surface that acts as a light diffuser, severely degrading the quality of the image of the beads. The sample was submersed in a watery dye solution to aid in visualizing the surface. The sample was placed in a two-photon fluorescence excitation microscope (TPM) with an SLM conjugated to the back-pupil plane of the microscope objective (see Appendix 4.5.1 for a description of the microscope setup). We performed three imaging experiments to demonstrate the feasibility and robustness of our technique. In the first experiment, we used conventional TPM (with no correction for scattering) to image the fluorescent beads. In the second experiment, we used current state-of-the-art feedback-based wavefront shaping to suppress the scattering introduced by the sample. We imaged the beads after applying the correction obtained from feedback-based wavefornt shaping. In the third experiment, the beads were imaged after applying correction obtained from our new model-based wavefront shaping method.

Figure 5.2a shows the maximum intensity projection of the 3D stack acquired using conventional TPM. We have combined thirteen 3D sub-stacks to cover the depth ( $z$-axis) range from $42 \mu \mathrm{m}$ to $325 \mu \mathrm{m}$ through the scattering layer. Each 3D sub-stack consists of 41 frames with a total volume of $26.2 \times 25.6 \times 21.7 \mu \mathrm{m}^{3}$. It is clear that the intensity of the image decreases rapidly as a function of distance from the scattering layer (located at $z=0$ ). Figure $5.2 \mathrm{~b}$ shows the maximum intensity projection after feedback-based wavefront shaping using a state-of-the-art Hadamard algorithm [16] with 256 input modes. For every 3D substack, only a single optimal wavefront was used, which was found by optimizing the feedback signal from a single fluorescent bead located at the center. It is clear from Fig. 5.2b that the intensities of the beads are higher than that in Fig. 5.2a. However, the method fails to optimize the focus after about a depth of $175 \mu \mathrm{m}$ where the SNR of the feedback signal is too low to find a correction wavefront..

For our new method, we first used the microscope to acquire a 3D intensity image of the sample surface, using the fluorescent dye in the water to visualize the rough interface. From this image, we constructed a 3D refractive index model of the PDMS-water interface. Next, we used a beam propagation method [17] for simulating light propagation from a point source located at the center of each 3D sub-stack (see Appendix 5.4 for technical details). 
(a) no correction

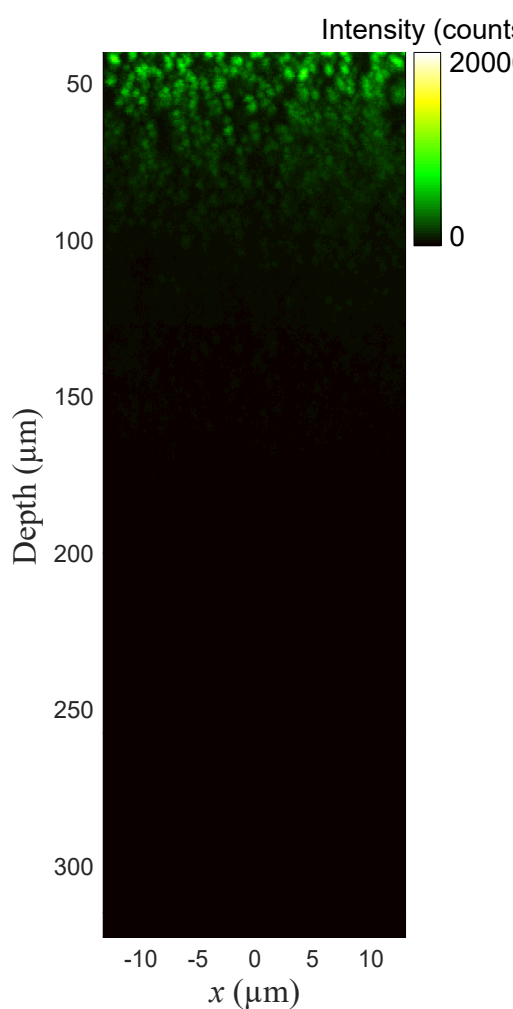

(b) feedback-based wavefront shaping
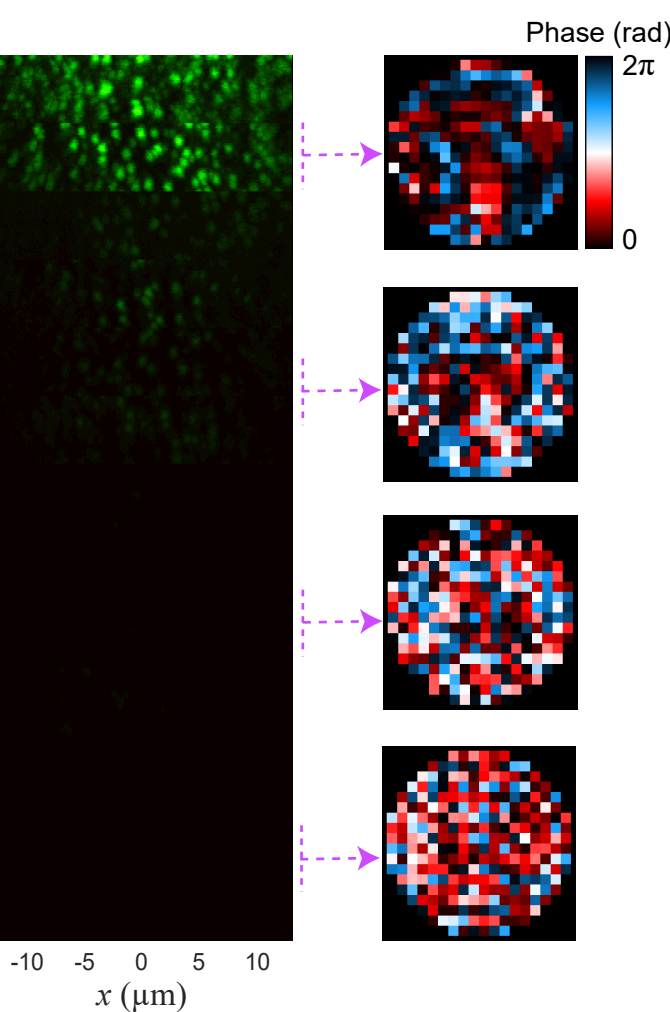

(c) model-based wavefront shaping

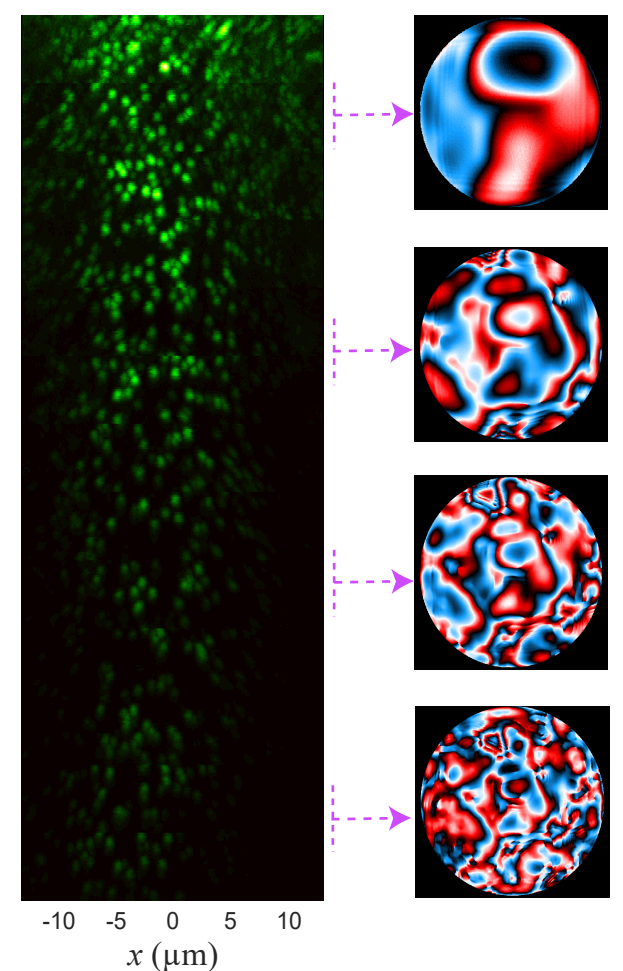

Figure 5.2: Scattering compensation using model-based wavefront shaping. Maximum intensity projection of the 3D image data acquired, (a) using conventional TPM without correction, (b) using feedback-based wavefront shaping and (c) using model-based wavefront shaping. The scattering surface is located at a depth of $z=0$. The wavefront corrections associated with four sub-stacks are also displayed. It is clear that model-based wavefront shaping works over the entire depth of interest, whereas the feedback-based method fails when noise dominates the feedback signal. 


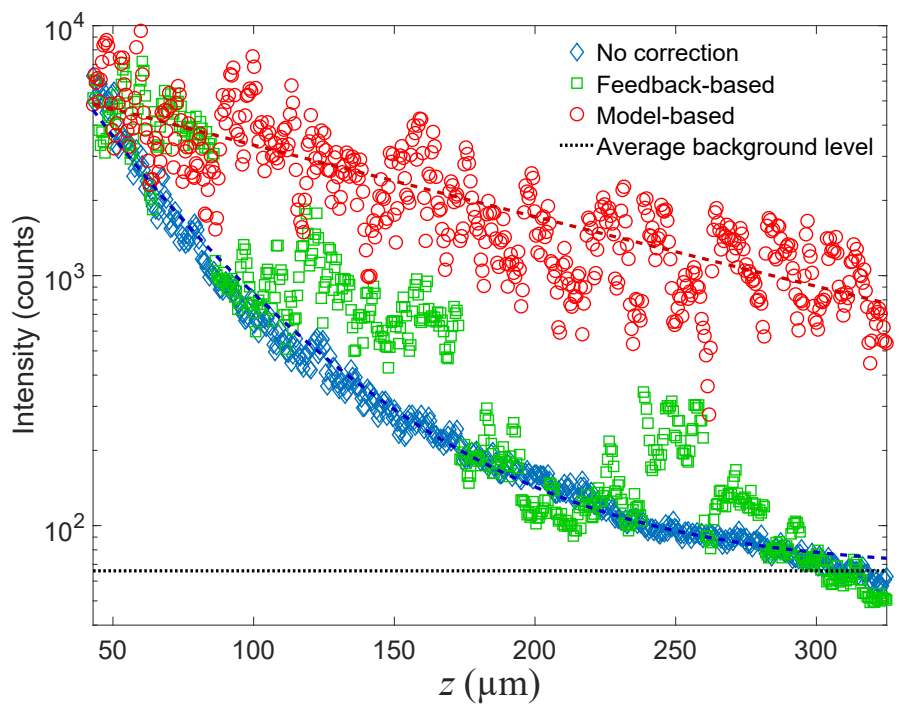

Figure 5.3: 2-photon fluorescence signal as a function of depth inside the sample. Blue diamonds, signal without correction; green squares, signal after feedback-based wavefront shaping; red circles, signal after model-based wavefront shaping. The dotted black line shows the average background level of the images. The dashed blue and red lines correspond to the theoretical expectations.

Figure 5.2c shows the results of model-based wavefront shaping. The fluorescent beads are visible all the way to the maximum depth of $325 \mu \mathrm{m}$, which is approximately twice the depth reached by feedback-based wavefront shaping. It is clear from the image that model-based wavefront shaping is consistently successful over the full depth range. Moreover, we can freely pick the position where we want the correction to be optimal (the center of each 3D sub-stack in our case), without relying on the presence of a guide star.

The optimized and computed wavefronts corresponding to four different sub-stacks are shown in Fig. 5.2b and c. It can be seen that model-based wavefront shaping finds an accurate representation of the correction wavefront, which becomes more complex with increasing depth. This result is a clear improvement over feedback-based wavefront shaping, where the resolution of the correction wavefront is limited by the SNR and the optimization time. Furthermore, it can be seen that deep inside the sample the intensity of the image degrades towards the edges in Fig. 5.2c. This is because, the scanning range of the optimized focus is limited to the range of memory effect [13]. The scanning range may be improved further by conjugating the SLM to the PDMS-water interface [18], or by finding different wavefront corrections for different positions in the sample.

Figure 5.3 depicts the two-photon signal as a function of depth before and after compensating for the scattering. Without compensation, the two-photon signal decreases rapidly as a function of depth. With increasing depth, the area of the scattering surface that is illuminated by the focusing beam increases quadratically, causing the excitation intensity in the focus to decrease as $z^{-2}$ [19]. When we also take into account an exponential attenuation, we find that the 2-photon signal $S$ decreases as $S \propto z^{-4} \exp -2 z / \ell$. The exponential decay can be attributed 
to the scattering and absorption by fluorescent beads and scattering by small imperfections (i.e. microscopic bubbles) in the bulk of the sample. A good fit to the experimental data is obtained with $\ell=295 \mu \mathrm{m}$ (blue dashed line in Fig. 5.3).

The feedback-based method successfully enhances the image intensity until about a depth of $175 \mu \mathrm{m}$ but fails to improve the focus at larger depths because of the drop in SNR. The maximum signal enhancement over the uncorrected case is less than a factor of five. On the other hand, model-based wavefront shaping works over the entire depth of the 3D image and shows a 21-fold increase in intensity at the deepest optimized point. It is to be noted that the signal slowly decreases with depth even after correction. As before, this decay is attributed to the absorption and scattering of light by the fluorescent beads in the bulk of the sample. The red dashed line in Fig. 5.3 shows that the remaining decay follows $S \propto \exp -2 z / \ell$, with $\ell=295 \mu \mathrm{m}$, as before. Therefore, we can conclude that $1 / z^{4}$ decay factor is compensated completely, and only the slow exponential decay due to scattering and absorption in the bulk remains.

\subsection{Conclusion}

This work introduces a new class of wavefront shaping methods combining TPM imaging and light propagation modeling to mitigate scattering in a robust way. We demonstrate the feasibility of our method in a proof of principle imaging experiment through a light diffusing layer. The main advantage of our technique over other methods is that it does not require any guide star for finding the optimal wavefront. Therefore, practical limitations associated with guide stars (like limited field of view, low SNR, number of optimized modes, photo-bleaching of the guide star, etc.) do not play a role at all.

The primary step in our method is the generation of a refractive index model. Whereas we used a single light-diffusing layer in this proof-of-concept experiment, in principle this technique can be extended to arbitrary turbid samples, as long as a refractive index model can be found. This model can be acquired by selectively labeling the fluorescence image data with known refractive index information, as we did here. We envision that other techniques, such as optical diffraction tomography, optical coherence tomography, ptychography, and structured illumination microscopy [20-23] may be used in order to relax the requirement for a priori knowledge about the sample.

To summarize, model-based wavefront shaping can perform near-perfect correction for scattering introduced by the diffusing layers in microscopy. It opens up a promising platform for integrating with aforementioned techniques, eliminating the need for guide stars altogether.

\subsection{Appendices}

\subsubsection{D Refractive index modeling}

We used TPM to image the interface between the PDMS diffuser and water. In order to visualize the surface, a dye of fluorescein was added to the water ( $1 \mathrm{mg} / \mathrm{mL}$, Sigma-Aldrich). We have acquired 60 TPM frames covering a volume of $500 \times 500 \times 60 \mu \mathrm{m}^{3}$. The voxel resolution of the $3 \mathrm{D}$ image has been chosen as $1.5 \times 1.5 \times 1 \mu^{3}$, such that the spatial variation of the 
(a)

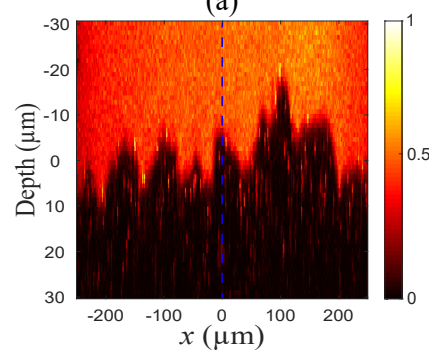

(b)

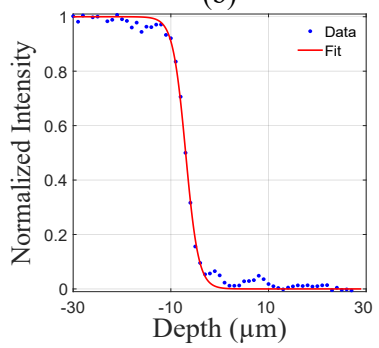

(c)

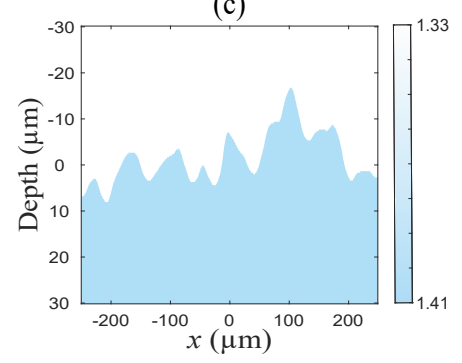

Figure 5.4: (a) A 2D cross-section of the acquired 3D TPM image of the PDMS-water interface. (b) An example intensity fit along the depth using a sigmoid function. (c) A 2D cross-section of the refractive index distribution model.

diffuser surface can be clearly imaged. Fig. 5.4a shows a 2D cross-section of the 3D image at $y=0$. Two separate regions can be identified in the figure. The bright region corresponds to fluorescein dye, whereas the dark region with localized high intensity spots corresponds to PDMS dispersed with fluorescent beads. A nonlinear fitting procedure has been implemented to automatically detect the PDMS-water interface. We first applied a low pass filter to the frames to suppress the high intensity spots. Next, for every $x, y$ position, sigmoid functions were fitted to the intensity data along the depth. For every fit, we computed the point of inflection, which was taken as the position of the PDMS-water interface. An example fit, of data taken along the blue dashed line in Fig. 5.4a, is shown in Fig. 5.4b. Finally, we assigned the refractive index values 1.33 and 1.41 to the regions of water and PDMS, respectively. A $2 \mathrm{D}$ cross-section of the refractive index model is shown in the Fig. 5.4c.

\subsubsection{Light propagation simulations}

We use a beam propagation method (BPM) adopting the angular spectrum method [17] to simulate the light propagation from a point source at a desired location inside the scattering sample. In principle, we simulate the recording step of a phase conjugation experiment. As the SLM is conjugated to the pupil plane of the objective lens, ideally what is required is a light propagation simulation through the sample, objective lens and other components of the microscope setup to the position of the SLM. For simplicity, we treat the microscope objective as a perfect lens, so that the field in the focal plane and the field at the pupil plane are Fourier pairs. The mapping from the pupil plane to the SLM plane is discussed in Appendix 5.4.3. We follow a simple procedure consisting of two steps to find the correction wavefront. The two steps consist of four sub-steps which are indicated by the blue-dashed rectangular regions in the Fig. 5.5.

1. As a first step, we propagate light from a point source inside the PDMS sample through scattering surface. In step 1a, we generate a spherical wavefront from a point source located at a distance $d$ inside the PDMS. A function of the form $\exp \left\{i \frac{2 \pi n_{\mathrm{PDMS}}}{\lambda}\left[\sqrt{d^{2}+x^{2}+y^{2}}\right]\right\}$, is used to model the diverging beam. $n_{\mathrm{PDMS}}$ is the refractive index of PDMS, $x$ and $y$ are the spatial coordinates of the electric field and $\lambda$ is the wavelength of the light. The electric field at plane $\mathrm{P} 1, E_{\mathrm{P} 1}(x, y)$, is apodized to match the numerical aperture of the microscope objective. 

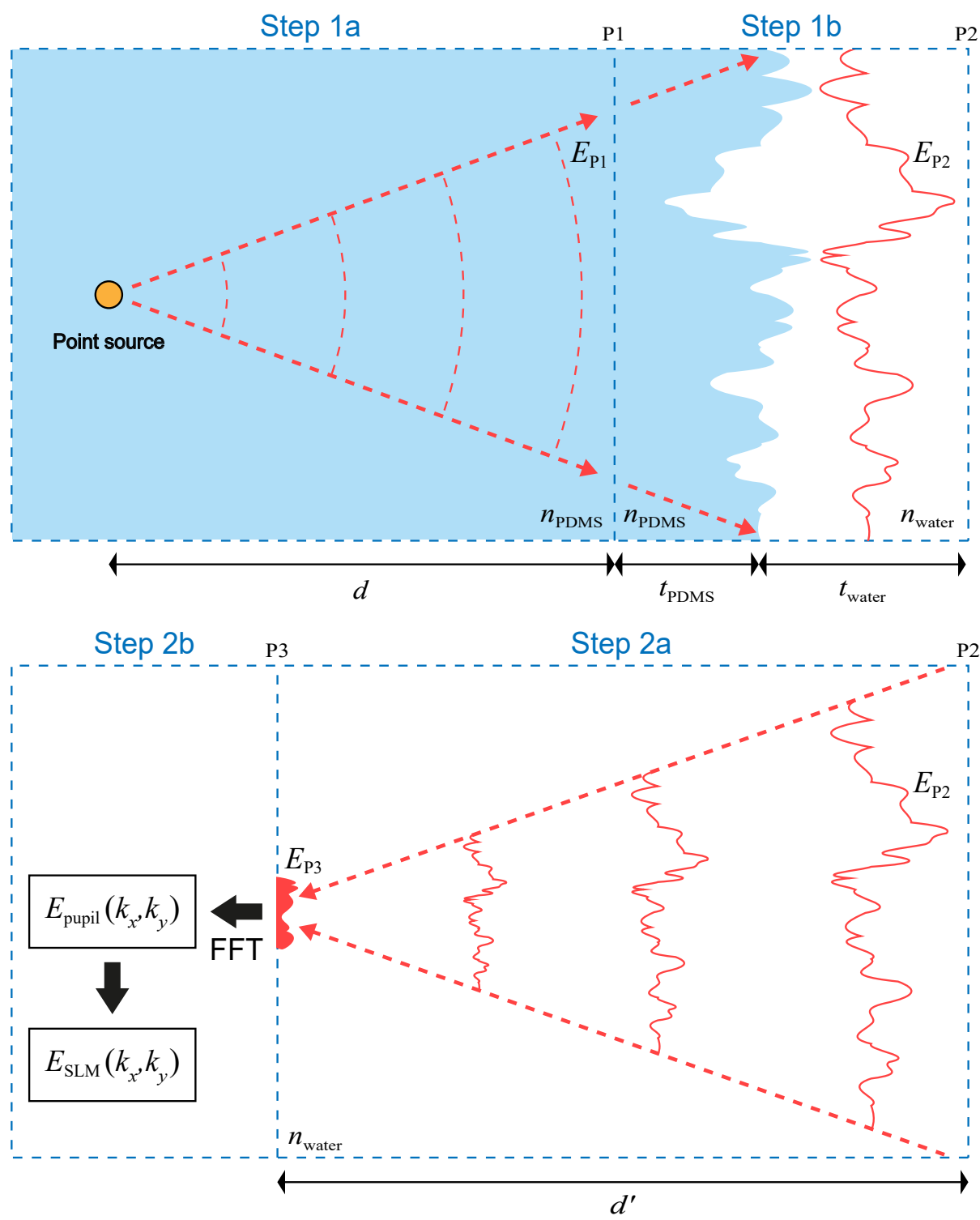

Figure 5.5: Simulation steps. Step 1a, Generation of a spherical wavefront (at plane P1) from a point source located at a distance $d$ inside the PDMS medium. Step 1b, BPM is used to propagate this wavefront to plane $\mathrm{P} 2$ through the reconstructed refractive index of the PDMSwater interface. Step 2a, The scattered field at P2 is digitally back propagated through water to the nominal focal plane $\mathrm{P} 3$. Step $2 \mathrm{~b}$, The field at $\mathrm{P} 3$ is propagated to the pupil plane of the microscope objective and finally mapped to the plane of the SLM. 
In step $1 \mathrm{~b}$, we use BPM to propagate the field $E_{\mathrm{P} 1}(x, y)$ through the reconstructed refractive index distribution of the interface between the PDMS and water. For BPM, we first convert the refractive index distribution with a total thickness of $60 \mu \mathrm{m}$ into 180 layers of equally-spaced, infinitely-thin phase plates providing an approximation to the $3 \mathrm{D}$ distribution [24]. The resulting computed field is $E_{\mathrm{P} 2}(x, y)$, which is located outside the sample at plane P2.

2. In the second step, we propagate the scattered field $E_{\mathrm{P} 2}(x, y)$ to the plane of the SLM. As our objective lens is designed to be immersed in water, we first propagate the scattered field $E_{\mathrm{P} 2}(x, y)$ back through water over a distance $d^{\prime}=\left(d+t_{\mathrm{PDMS}}\right) \frac{n_{\mathrm{water}}}{n_{\mathrm{PDMS}}}+t_{\text {water }}$ to the plane P3 (step 2a). $n_{\text {water }}$ is the refractive index of water, $t_{\text {PDMS }}$ and $t_{\text {water }}$ are mean thicknesses of PDMS and water media calculated from the constructed refractive index distribution of the interface. Next, the computed field $E_{\mathrm{P} 3}(x, y)$ is propagated to the pupil plane with a twodimensional Fourier transform to get $E_{\text {pupil }}\left(k_{x}, k_{y}\right)$ (step $2 b$ ). Finally, we obtain $E_{\mathrm{SLM}}\left(k_{x}, k_{y}\right)$ by mapping $E_{\text {pupil }}\left(k_{x}, k_{y}\right)$ to the plane of the SLM (see Appendix 5.4.3).

In the experiment, $E_{\mathrm{SLM}}\left(k_{x}, k_{y}\right)$ is conjugated, and its phase is displayed on the SLM, resulting in a sharp focus at the position of the point source. Using a standard desktop PC, these simulations took less than 30 seconds to find the optimal wavefront.

\subsubsection{Calibration measurements for mapping SLM pixels to pupil plane}

In order to successfully perform model-based wavefront shaping, the SLM pixels must be accurately mapped to the coordinates of the computer simulation. This is done by a sequence of calibration measurements. To go through the calibration procedure, let us first define the coordinates in the sample space as $(x, y)$ in $\mu \mathrm{m}$, the coordinates in the TPM image space as $(X, Y)$ in frame pixels, the coordinates in SLM space as $(u, v)$ in SLM pixels and the spatial frequencies in the pupil plane as $\left(k_{x}, k_{y}\right)$ in $\mathrm{rad} / \mu \mathrm{m}$. Fig. 5.6 shows a block diagram with the different geometrical spaces and the transformation matrices connecting them. TPM imaging is carried out by scanning the angle of the incident beam using galvo mirrors. It is important to note that, as the galvo mirrors are scanning, the beam is standing still on the SLM and only the angle of incidence is changing. The steps in the calibration procedure are as follows:

Step 1: We image the beam profile on the SLM with the camera (see Appendix 4.5.1). From the camera image, we determine the center coordinates of the laser beam on the SLM. In the alignment process, we centered the beam with respect to the pupil plane of the objective lens.

Step 2: We calculate the field of view and the resolution of the TPM frame. We first calibrated the sample 2D stage (Zaber, ASR series microscope stage) with a resolution target (Thorlabs R1L3S6P). The two lateral stages in the Zaber are assumed to move orthogonally. In order to find the transformation matrix, which converts pixel coordinates in the TPM frame to the coordinates in sample space, we displaced the sample with the Zaber stage. As the calibration sample, we used a 2D planar distribution of fluorescent beads made on top of a microscope slide. Initially an image of the sample is captured and saved as a reference frame. Then we captured two TPM frames with horizontal and vertical displacements using the lateral stages. We calculated the cross-correlations of the displaced frames with the initial reference frame. The peak positions in the cross-correlations are used to calculate the shifts in the frame pixels. In mathematical form, a transformation matrix $M$ can be obtained by 


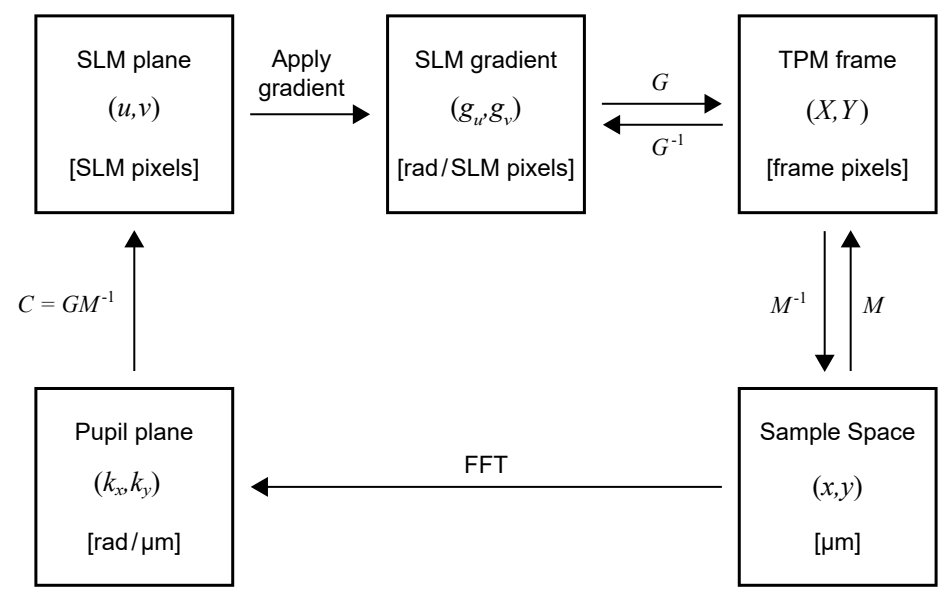

Figure 5.6: Block diagram showing the different geometrical spaces and the corresponding transformation matrices.

inverting the following relation:

$$
\left(\begin{array}{cc}
\Delta X_{1} & \Delta X_{2} \\
\Delta Y_{1} & \Delta Y_{2}
\end{array}\right)=M\left(\begin{array}{ll}
\Delta x & 0 \\
0 & \Delta y
\end{array}\right),
$$

where $\Delta X_{1}, \Delta Y_{1}, \Delta X_{2}$ and $\Delta Y_{2}$ are image shifts (in frame pixels) observed in the TPM frame. $\Delta x$ and $\Delta y$ are the horizontal and vertical displacements (in $\mu \mathrm{m}$ ) applied on the Zaber stage. The unit of the transformation matrix $M$ is [frame pixels $/ \mu \mathrm{m}$ ].

Step 3: The next step is to find the transformation matrix that relates the SLM coordinates to the sample space coordinates. Initially, we captured the reference frame of the calibration sample. Then we captured two TPM frames after applying gradients along the $u$-axis and the $v$-axis on the SLM. Since the SLM is conjugated to the pupil of the microscope objective, these gradients respectively cause horizontal and vertical shifts of the recorded image. We calculated the cross-correlations of the displaced frames with the initial reference frame. The peak positions in the cross-correlations are used to calculate the shifts in the frame pixels. The transformation matrix $G$ can be obtained from the following relation:

$$
\left(\begin{array}{ll}
\Delta X_{1} & \Delta X_{2} \\
\Delta Y_{1} & \Delta Y_{2}
\end{array}\right)=G\left(\begin{array}{ll}
g_{u} & 0 \\
0 & g_{v}
\end{array}\right) .
$$

$g_{u}$ and $g_{v}$ are the gradients (in rad/SLM pixels) applied on the SLM. The unit of $G$ is [frame pixels $\times$ SLM pixels/rad].

Step 4: Finally, we need to map the Fourier-space coordinates $k_{x}, k_{y}$ of the simulation to SLM pixels. This is done by combining the two transformation matrices $M$ and $G$ as follows,

$$
C=G M^{-1} \text {. }
$$

The unit of conversion matrix, $C$ is [SLM pixels $\times \mu \mathrm{m} / \mathrm{rad}]$. By applying this conversion matrix on the $k$-space coordinates $\left(k_{x}, k_{y}\right)$ in $\mathrm{rad} / \mu \mathrm{m}$ of the simulation space, we find the SLM coordinates, 


$$
\left(\begin{array}{l}
u \\
v
\end{array}\right)=C\left(\begin{array}{l}
k_{x} \\
k_{y}
\end{array}\right) .
$$

\section{Acknowledgements}

This work was supported by the European Research Council (ERC-2016-StG-678919). We would like to thank Tzu Lun Ohn for providing the protocol for the sample preparation.

\section{Bibliography}

[1] J. Kubby, S. Gigan, and M. Cui, Wavefront Shaping for Biomedical Imaging, Advances in Microscopy and Microanalysis (Cambridge University Press) (2019).

[2] A. P. Mosk, A. Lagendijk, G. Lerosey, and M. Fink, "Controlling waves in space and time for imaging and focusing in complex media", Nat. Photonics 6, 283-292 (2012).

[3] Z. Yaqoob, D. Psaltis, M. S. Feld, and C. Yang, "Optical phase conjugation for turbidity suppression in biological samples”, Nat. Photonics 2, 110-115 (2008).

[4] I. M. Vellekoop and A. P. Mosk, "Focusing coherent light through opaque strongly scattering media", Opt. Lett. 32, 2309-2311 (2007).

[5] R. Horstmeyer, H. Ruan, and C. Yang, "Guidestar-assisted wavefront-shaping methods for focusing light into biological tissue”, Nat. Photonics 9, 563-571 (2015).

[6] C.-L. Hsieh, Y. Pu, R. Grange, and D. Psaltis, "Digital phase conjugation of second harmonic radiation emitted by nanoparticles in turbid media", Opt. Express 18, 1228312290 (2010).

[7] I. M. Vellekoop, M. Cui, and C. Yang, "Digital optical phase conjugation of fluorescence in turbid tissue", Appl. Phys. Lett. 101, 081108 (2012).

[8] C. Ma, X. Xu, Y. Liu, and L. V. Wang, “Time-reversed adapted-perturbation (TRAP) optical focusing onto dynamic objects inside scattering media”, Nat. Photonics 8, 931936 (2014).

[9] E. H. Zhou, H. Ruan, C. Yang, and B. Judkewitz, "Focusing on moving targets through scattering samples", Optica 1, 227-232 (2014).

[10] O. Katz, E. Small, Y. Guan, and Y. Silberberg, "Noninvasive nonlinear focusing and imaging through strongly scattering turbid layers", Optica 1, 170-174 (2014).

[11] C. Moretti and S. Gigan, "Readout of fluorescence functional signals through highly scattering tissue", Nat. Photonics 14, 361-364 (2020).

[12] G. Osnabrugge, L. V. Amitonova, and I. M. Vellekoop, "Blind focusing through strongly scattering media using wavefront shaping with nonlinear feedback", Opt. Express 27, 11673-11688 (2019). 
[13] G. Osnabrugge, R. Horstmeyer, I. Papadopoulos, B. Judkewitz, and I. Vellekoop, "Generalized optical memory effect”, Optica 4, 886-892 (2017).

[14] X. Xu, H. Liu, and L. V. Wang, “Time-reversed ultrasonically encoded optical focusing into scattering media”, Nat. Photonics 5, 154-157 (2011).

[15] Y. M. Wang, B. Judkewitz, C. A. DiMarzio, and C. Yang, "Deep-tissue focal fluorescence imaging with digitally time-reversed ultrasound-encoded light", Nat. Commun. 3, 928 (2012).

[16] X. Tao, T. Lam, B. Zhu, Q. Li, M. R. Reinig, and J. Kubby, “Three-dimensional focusing through scattering media using conjugate adaptive optics with remote focusing (caorf)", Opt. Express 25, 10368-10383 (2017).

[17] J. Goodman, Introduction to Fourier Optics, McGraw-Hill physical and quantum electronics series (W. H. Freeman) (2005).

[18] J. Mertz, H. Paudel, and T. G. Bifano, "Field of view advantage of conjugate adaptive optics in microscopy applications", Appl. Opt. 54, 3498-3506 (2015).

[19] H. G. Booker, J. A. Ratcliffe, D. H. Shinn, and W. L. Bragg, "Diffraction from an irregular screen with applications to ionospheric problems", Philos. Trans. R. Soc. A 242, 579-607 (1950).

[20] W. Choi, C. Fang-Yen, K. Badizadegan, S. Oh, N. Lue, R. R. Dasari, and M. S. Feld, "Tomographic phase microscopy", Nat. Methods 4, 717-719 (2007).

[21] T. Callewaert, J. Dik, and J. Kalkman, "Segmentation of thin corrugated layers in highresolution oct images”, Opt. Express 25, 32816-32828 (2017).

[22] S. Chowdhury, M. Chen, R. Eckert, D. Ren, F. Wu, N. Repina, and L. Waller, "Highresolution $3 \mathrm{~d}$ refractive index microscopy of multiple-scattering samples from intensity images", Optica 6, 1211-1219 (2019).

[23] S. Chowdhury, W. J. Eldridge, A. Wax, and J. Izatt, "Refractive index tomography with structured illumination”, Optica 4, 537-545 (2017).

[24] J. Yang, J. Li, S. He, and L. V. Wang, "Angular-spectrum modeling of focusing light inside scattering media by optical phase conjugation”, Optica 6, 250-256 (2019). 


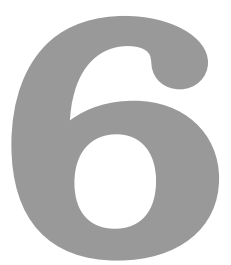

\section{A convergent Born series for solving the inhomogeneous Helmholtz equation in arbitrarily large media}

We present a fast method for numerically solving the inhomogeneous Helmholtz equation. Our iterative method is based on the Born series, which we modified to achieve convergence for scattering media of arbitrary size and scattering strength. Compared to pseudospectral time-domain simulations, our modified Born approach is two orders of magnitude faster and nine orders of magnitude more accurate in benchmark tests in 1, 2, and 3-dimensional systems.

\subsection{Introduction}

The Helmholtz equation, the time-independent form of the scalar wave equation, appears in many fields of physics ranging from electron scattering to seismology. Additionally, this equation describes electromagnetic wave propagation in 2-dimensional systems, and it is often used as a scalar approximation to light propagation in 3-dimensional scattering media [1].

A great variety of approaches are available for solving the Helmholtz equation in inhomogeneous media [2]. For most numerical methods, the equation is discretized using the finite difference [3] or the finite element $[4,5]$ approximation. The corresponding system of linear equations can be solved using direct matrix inversion methods $[6,7]$, but these methods are ineffective for solving large inhomogeneous systems. More efficient methods exist, such as the category of Krylov subspace methods [8] extended with multigrid [9, 10] or domain

This chapter has been published as: [G. Osnabrugge, S. Leedumrongwatthanakun and I.M. Vellekoop, J. Comput. Phys. 322, 113-124 (2016)]. 
decomposition methods $[11,12]$. However, solving the inhomogeneous Helmholtz equation for large systems remains a computationally challenging task [13].

For the simulation of light propagation, finite-difference time-domain methods (FDTD) $[14,15]$ and pseudospectral time domain methods (PSTD) [16-18] are used the most [19]. Using these time-domain methods, the steady-state solution is found by replacing the source term with a periodically oscillating source and running the simulations until steady state is reached. However, the finite difference approximations required for these methods are only exact in the limit of an infinitely small step size, resulting in a trade-off between accuracy and speed.

A completely different class of methods is formed by volume integral methods utilizing the Green's function [20-23]. From Green's function theorem, the well-known Born series can be derived [1]. The Born series has long been used to solve the scattering problem for small particles with a low scattering potential. However, the series diverges for larger structures, or structures with a high scattering contrast [24].

Here we present a modified version of the Born series, that unconditionally converges to the exact solution of the Helmholtz equation for arbitrarily large structures with an arbitrarily high scattering potential. Our method converges rapidly and monotonically to the exact solution, and is several orders of magnitude faster than PSTD.

\subsection{Traditional Born series}

First, we will introduce our modified Born series, and discuss the convergence criteria and other characteristics. Then, we compare the accuracy and run-time of our method to that of PSTD for 1, 2, and 3-dimensional homogeneous and inhomogeneous media. A proof of convergence is given in Appendix 6.7.1 and the boundary conditions for our experiments are presented in Appendix 6.7.2.

First, the inhomogeneous Helmholtz equation is considered

$$
\nabla^{2} \psi(\mathbf{r})+k(\mathbf{r})^{2} \psi(\mathbf{r})=-S(\mathbf{r})
$$

where $\psi(\mathbf{r})$ represents the field at position $\mathbf{r} \in \mathbb{R}^{D}$, with $D$ representing the dimensionality of the problem. $S(\mathbf{r})$ is the source term and $k(\mathbf{r})$ is the wavenumber. The scattering potential is defined as $V(\mathbf{r}) \equiv k(\mathbf{r})^{2}-k_{0}^{2}-i \varepsilon$ to find

$$
\nabla^{2} \psi(\mathbf{r})+\left(k_{0}^{2}+i \varepsilon\right) \psi(\mathbf{r})=-V(\mathbf{r}) \psi(\mathbf{r})-S(\mathbf{r}) .
$$

Typically, one chooses $k_{0}$ as the background potential and $\varepsilon$ infinitesimally small [20]. However, the solution of Eq. (6.2) does not depend on the choice for $k_{0}$ and $\varepsilon$ at all. Using the Green's function theorem, the solution can be written as

$$
\psi(\mathbf{r})=\int g_{0}\left(\mathbf{r}-\mathbf{r}^{\prime}\right)\left[V\left(\mathbf{r}^{\prime}\right) \psi\left(\mathbf{r}^{\prime}\right)+S\left(\mathbf{r}^{\prime}\right)\right] d \mathbf{r}^{\prime},
$$

where the Green's function $g_{0}$ is defined as the solution to

$$
\nabla^{2} g_{0}(\mathbf{r})+\left(k_{0}^{2}+i \varepsilon\right) g_{0}(\mathbf{r})=-\delta(\mathbf{r}) .
$$


The Green's function can easily be found in Fourier transformed coordinates $\mathbf{p}$, which is given by $\tilde{g_{0}}(\mathbf{p})=1 /\left(|\mathbf{p}|^{2}-k_{0}^{2}-i \varepsilon\right)$. Eq. (6.3) can now be simplified to the matrix form

$$
\psi=G V \psi+G S
$$

where the convolution with $g_{0}$ is replaced by a single operator $G \equiv F^{-1} \tilde{g_{0}}(\mathbf{p}) F$, with $F$ and $F^{-1}$ the forward and inverse Fourier transforms, respectively. $V$ is a diagonal matrix, and $S$ and $\psi$ are vectors. Eq. (6.5) can be expanded recursively to arrive at the traditional Born series

$$
\psi_{\text {Born }}=[1+G V+G V G V+\ldots] G S .
$$

Note that this series only converges to $\psi$ if the spectral radius $\rho$ of operator $G V$ is less than unity. The Born series has proven to be successful for solving the Helmholtz equation for small scattering structures with weak scattering potential [25]. However, it is well known that there are serious constraints on the convergence of the series for larger and stronger scattering structures, limiting the usefulness of this approach [24].

\subsection{Modified Born series}

\subsubsection{Convergent Born series}

Here we show that the series can be made convergent for any arbitrary scattering potential by choosing a suitable preconditioner $\gamma$. Applying this preconditioner to Eq. (6.5) gives

$$
\begin{gathered}
\gamma \psi=\gamma G V \psi+\gamma G S \\
\psi=M \psi+\gamma G S
\end{gathered}
$$

with

$$
M \equiv \gamma G V-\gamma+1
$$

We now choose

$$
\begin{aligned}
\gamma(\mathbf{r}) & =\frac{i}{\varepsilon} V(\mathbf{r}), \\
\varepsilon & \geq \max _{\mathbf{r}}\left|k(\mathbf{r})^{2}-k_{0}^{2}\right| .
\end{aligned}
$$

As we show in Appendix 6.7.1, this combination of $\gamma$ and $\varepsilon$ ensures that $\rho(M)<1$, meaning that Eq. (6.8) can be expanded recursively to arrive at our modified Born series

$$
\psi=\left[1+M+M^{2}+M^{3}+\ldots\right] \gamma G S,
$$

or, in iterative form

$$
\psi_{k+1}=M \psi_{k}+\gamma G S
$$

with $\psi_{0}=\gamma G S$. This iteration converges to the exact solution of the Helmholtz equation for arbitrarily large media. 


\subsubsection{Interpretation}

By introducing $\varepsilon$ and the preconditioner $\gamma$, we are able to ensure the convergence of the Born series. In order to gain an intuitive understanding of our algorithm, we first analyze the role of $\varepsilon$. As can be seen from Eq. (6.4), $\varepsilon$ introduces an imaginary component to the wavevector of the background medium. As a result, the Green's function in a homogeneous medium decays exponentially with distance. For instance, in three dimensions the solution to Eq. (6.4) is

$$
g_{0}(\mathbf{r})=\frac{e^{i|\mathbf{r}| \sqrt{k_{0}^{2}+i \varepsilon}}}{4 \pi|\mathbf{r}|} .
$$

The exponential decay makes that the total amount of energy represented by the Green's function is finite and localized.

The term $i \varepsilon$ in the background potential is compensated exactly by an imaginary term in the scattering potential $V$, such that the final solution of the Helmholtz equation remains the same. A physical interpretation of this construct is that the background medium is lossy, and the scattering potential compensates this loss by an equal amount of gain. As a result, even a homogeneous medium has a non-zero scattering potential.

Fig. 6.1a-c show the solution for a homogeneous medium after 20, 40, and 60 iterations respectively. It can be seen that the solution expands with each iteration. This expansion should not be confused with time-resolved propagation, since the algorithm is only solving a time-independent wave equation. Still, it is useful to analyze this 'pseudo-propagation' effect since it gives an indication of how many iterations are required to cover a homogeneous medium of a given size. We define the pseudo-propagation distance as the distance traveled by a 1-dimensional wave packet $\Psi_{0}(x) \equiv A(x) \exp \left(i k_{0} x\right)$, with $x$ as the spatial dimension and $A$ as a positive-valued envelope function. The center of the wave packet is defined as

$$
\left\langle x_{0}\right\rangle \equiv \frac{\int x A(x) d x}{\int A(x) d x}=\frac{\int x \Psi_{0}(x) e^{-i k_{0} x} d x}{\int \Psi_{0}(x) e^{-i k_{0} x} d x}=\left.\frac{d \tilde{\Psi}_{0}\left(p_{x}\right)}{\tilde{\Psi}_{0}\left(p_{x}\right) i d p_{x}}\right|_{p_{x}=k_{0}}
$$

with $\tilde{\Psi}_{0}$ denoting the Fourier transform of $\Psi_{0}$. After a single iteration the packet has transformed into

$$
\Psi_{1}(x)=[\gamma G V-\gamma+1] \Psi_{0}(x)
$$

For a homogeneous medium $(V=-i \varepsilon$, and $\gamma=i V / \varepsilon=1)$

$$
\tilde{\Psi}_{1}\left(p_{x}\right)=\left[\frac{-i \varepsilon}{p_{x}^{2}-k_{0}^{2}-i \varepsilon}\right] \tilde{\Psi}_{0}\left(p_{x}\right) .
$$

The center of the shifted wave packet $\Psi_{1}$ is at

$$
\left\langle x_{1}\right\rangle=\left.\frac{d \tilde{\Psi}_{1}\left(p_{x}\right)}{\tilde{\Psi}_{1}\left(p_{x}\right) i d p_{x}}\right|_{p_{x}=k_{0}}=2 k_{0} / \varepsilon+\left\langle x_{0}\right\rangle,
$$

with $\left\langle x_{0}\right\rangle$ the center of the original wave packet. In words, each iteration moves the wave packet over a distance $2 k_{0} / \varepsilon$. This behavior is illustrated in Fig. 6.1d. The plot shows the 

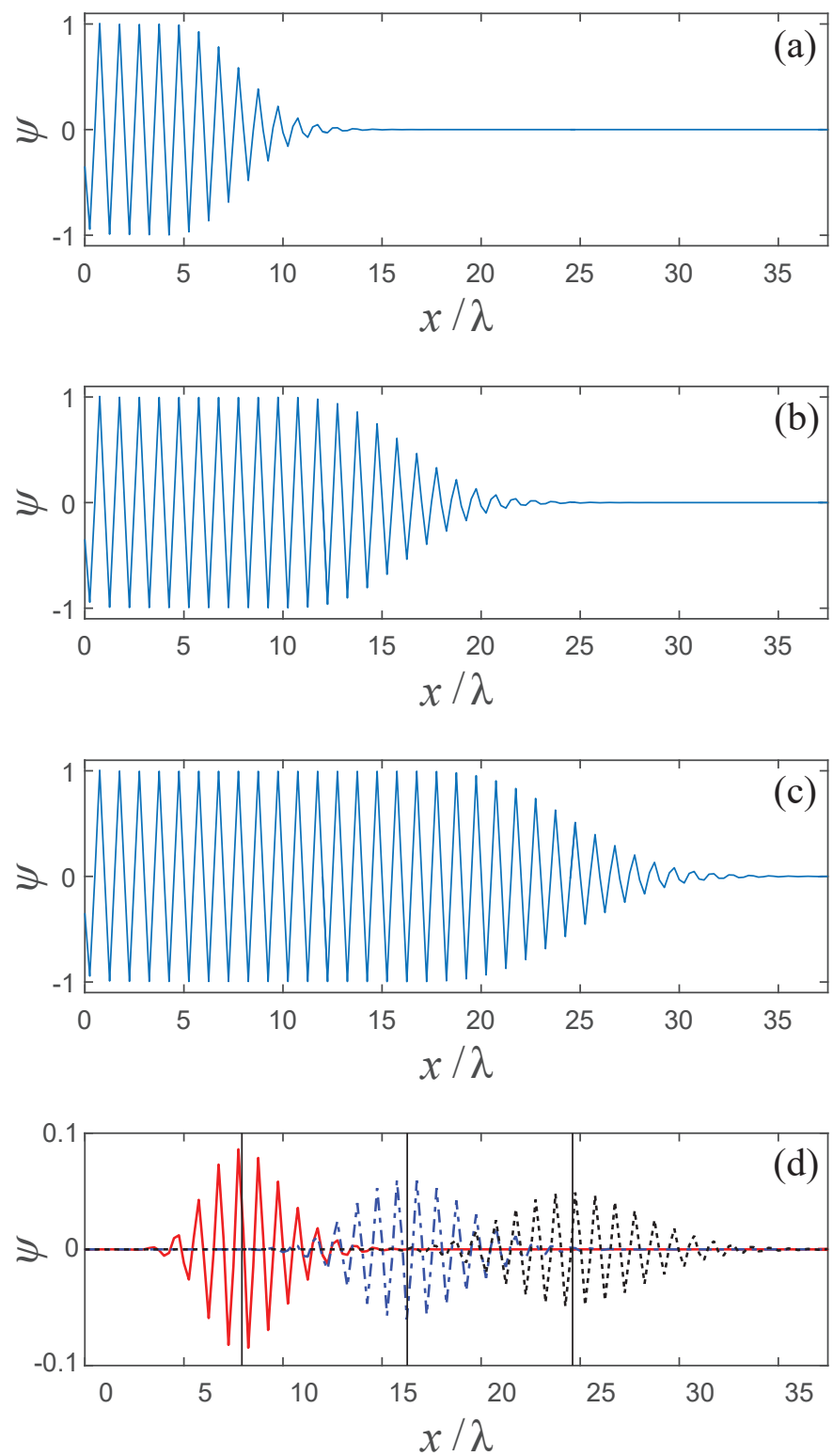

Figure 6.1: Solution for wave propagation in a homogeneous medium after (a) 20, (b) 40, and (c) 60 iterations respectively. (d) The field added during iteration 20 (solid red), iteration 40 (dashed blue), and iteration 60 (dotted black). The vertical lines show the theoretical positions of the differential contributions, calculated using Eq. (6.18). In all simulations $\varepsilon=0.8 k_{0}^{2}$. 


\begin{tabular}{l|c|c|c|c} 
& symbol & Modified Born & PSTD [16] & FDTD [14] \\
\hline grid spacing & $\Delta x$ & $\leq \lambda_{m} / 2$ & $\leq \lambda_{m} / 2$ & $\leq \lambda_{m} / 8$ \\
accuracy & & machine precision & $O\left(\Delta t^{2}\right)$ & $O\left(\Delta t^{2} \Delta x^{2}\right)$ \\
total grid points & $N_{\text {tot }}$ & $\geq\left(k_{m} L / \pi\right)^{D}$ & $\geq\left(k_{m} L / \pi\right)^{D}$ & $\geq\left(4 k_{m} L / \pi\right)^{D}$ \\
FLOPs / iterations & $\mathrm{F}$ & $O\left(N_{t o t} \log N_{t o t}\right)$ & $O\left(N_{t o t} \log N_{t o t}\right)$ & $O\left(N_{t o t}\right)$ \\
& & $O\left(k_{m}^{D} \log k_{m}^{D}\right)$ & $O\left(k_{m}^{D} \log k_{m}^{D}\right)$ & $O\left(k_{m}^{D}\right)$ \\
time step & $\Delta t$ & $\mathrm{n} / \mathrm{a}$ & $\leq \frac{2 \Delta x}{\pi c \sqrt{D}}$ & $\leq \frac{\Delta x}{c \sqrt{D}}$ \\
propagation & $\Delta r$ & $2 k_{0} / \varepsilon \leq \frac{2}{k_{m} v}$ & $c \Delta t \leq \frac{2}{k_{m} \sqrt{D}}$ & $c \Delta t \leq \frac{\pi}{4 k_{m} \sqrt{D}}$ \\
total FLOPs & & $O\left(v k_{m}^{D+1} \log k_{m}^{D}\right)$ & $O\left(\sqrt{D} k_{m}^{D+1} \log k_{m}^{D}\right)$ & $O\left(\sqrt{D} k_{m}^{D+1}\right)$
\end{tabular}

Table 6.1: Key parameters for our modified Born approach, the PSTD and FDTD numerical methods. Here the largest wavenumber $k_{m} \equiv \max _{\mathbf{r}}|k(\mathbf{r})|$, the shortest wavelength $\lambda_{m} \equiv 2 \pi / k_{m}$ and $\mathrm{L}$ is the length of the system. The scattering contrast $v \equiv\left(\frac{k_{m}}{k_{0}}-\frac{k_{0}}{k_{m}}\right)$.

differential contributions of the 20th, 40th, and 60th iteration. Clearly, each iteration only modifies the field in a finite volume, and the solution expands at a speed of $2 k_{0} / \varepsilon$ per iteration. Eq. (6.18) shows that $\varepsilon$ should be chosen as low as possible to ensure rapid convergence. Since the minimum value of $\varepsilon$ is given by Eq. (6.11), our method will be faster for structures with a lower contrast. Therefore, we let $k_{0}^{2}=\left(\min _{\mathbf{r}} \operatorname{Re}\left\{k^{2}(\mathbf{r})\right\}+\max _{\mathbf{r}} \operatorname{Re}\left\{k^{2}(\mathbf{r})\right\}\right) / 2$ in order to minimize $\varepsilon$.

The pseudo-propagation speed can be compared to the actual propagation speed in other methods such as PSTD and FDTD. Table 6.1 summarizes the key characteristics of our method compared to PSTD and FDTD. Because of the required finite difference approximations, the accuracy of FDTD is proportional to both $\Delta t^{2}$ and $\Delta x^{2}$, and the accuracy of PSTD is proportional to $\Delta t^{2}$, whereas the modified Born method is only restricted by machine precision. By comparing the (pseudo-)propagation speed, it can be seen that if $2 k_{0} / \varepsilon>c \Delta t$ or

$$
\left(\frac{k_{m}}{k_{0}}-\frac{k_{0}}{k_{m}}\right)<\sqrt{D},
$$

our method is always faster than PSTD. For $D=3$, this condition corresponds with $k_{m} / k_{0}<$ 2.2. For example, if the scalar wave approximation is used to simulate light propagation in biological tissue, with a refractive index between 1.33 and 1.45 , our exact method 'propagates' at a speed of 2.7 wavelengths per iteration, which is a factor 21 faster than a PSTD simulation ran at the lowest accuracy possible.

\subsection{Implementation}

We implemented the modified Born algorithm in Matlab. The field $\psi$, and potential map $V$ are discretized on a regular 2-dimensional grid with a grid spacing of $\Delta x=\lambda / 4$, with $\lambda$ an arbitrarily chosen wavelength of 1 distance unit. The field $\psi$, the potential $V$, and the 
pre-computed $\tilde{g}_{0}$ are stored in 2-dimensional arrays. Our iterative algorithm is implemented as

$$
\psi_{k+1}(\mathbf{r})=\psi_{k}(\mathbf{r})-\frac{i}{\varepsilon} V(\mathbf{r})\left(\psi_{k}(\mathbf{r})-\operatorname{ifft}_{2}\left[\tilde{g_{0}}(\mathbf{p}) \mathrm{fft}_{2}\left[V(\mathbf{r}) \psi_{k}(\mathbf{r})+S(\mathbf{r})\right]\right]\right),
$$

where $\mathrm{fft}_{2}$ and ifft 2 are the forward and inverse 2-dimensional fast Fourier transform operators, index $k$ is the iteration number, and $S$ is the source. All multiplications are point-wise multiplications.

In order to compare the accuracy and efficiency of our approach with that of PSTD, we used a PSTD algorithm to solve the time-dependent scalar wave equation for a time-harmonic source [26]

$$
\nabla^{2} \psi(\mathbf{r}, t)-\frac{1}{c^{2}(\mathbf{r})} \frac{\partial^{2} \psi(\mathbf{r}, t)}{\partial t^{2}}-\frac{\sigma(\mathbf{r})}{c^{2}(\mathbf{r})} \frac{\partial \psi(\mathbf{r}, t)}{\partial t}=-S(\mathbf{r}) e^{-i \omega t},
$$

with $\omega$ the angular frequency of the source, $c(\mathbf{r})$ the wave velocity, and $\sigma(\mathbf{r})$ the damping rate. We choose the time units to have unit wave velocity, such that $\omega=2 \pi / \lambda$. By Fourier transforming Eq. (6.21) with respect to time, we find back the Helmholtz equation

$$
\nabla^{2} \psi(\mathbf{r})+\frac{\omega^{2}}{c^{2}(\mathbf{r})} \psi(\mathbf{r})+\frac{i \omega \sigma(\mathbf{r})}{c^{2}(\mathbf{r})} \psi(\mathbf{r})=-S(\mathbf{r}) .
$$

This result shows that we can solve the Helmholtz equation using PSTD by choosing $c^{2}(\mathbf{r})=$ $\omega^{2} / \operatorname{Re}\left\{k^{2}(\mathbf{r})\right\}$ and $\sigma(\mathbf{r})=\omega \operatorname{Im}\left\{k^{2}(\mathbf{r})\right\} / \operatorname{Re}\left\{k^{2}(\mathbf{r})\right\}$, and propagating the simulated wave until a steady state is reached.

We use the following PSTD algorithm [26] to solve the time-dependent scalar wave equation in presence of attenuation

$$
\psi_{k+1}(\mathbf{r})=C^{(1)}(\mathbf{r}) \psi_{k-1}(\mathbf{r})+C^{(2)}(\mathbf{r}) \psi_{k}(\mathbf{r})+C^{(3)}(\mathbf{r})\left(\operatorname{ifft}_{2}\left[-|\mathbf{p}|^{2} \mathrm{fft}_{2}\left[\psi_{k}(\mathbf{r})\right]\right]+S(\mathbf{r})\right),
$$

where coefficient matrices $C^{(1)}, C^{(2)}$ and $C^{(3)}$ are pre-calculated:

$$
C^{(1)}(\mathbf{r})=\frac{\sigma(\mathbf{r}) \Delta t-2}{\sigma(\mathbf{r}) \Delta t+2}, \quad C^{(2)}(\mathbf{r})=\frac{4}{\sigma(\mathbf{r}) \Delta t+2}, \quad C^{(3)}(\mathbf{r})=\frac{2 c^{2}(\mathbf{r}) \Delta t^{2}}{\sigma(\mathbf{r}) \Delta t+2} .
$$

Since PSTD uses a finite-difference scheme to approximate the time derivative, the accuracy of the method will depend on $\Delta t$. In the limit of $\Delta t \rightarrow 0$, PSTD should converge to the exact solution that is found with our modified Born approach.

We have the option to either use absorbing boundaries or periodic boundary conditions. Choosing a proper absorbing boundary condition is not trivial as any residual reflection or transmission will affect the accuracy of the simulation. We found that for our high-accuracy simulations a good trade-off between layer thickness and accuracy was achieved with the boundaries described in Appendix 6.7.2.

\subsection{Results}

\subsubsection{Homogeneous medium}

In the first numerical experiment, we simulated wave propagation through a homogeneous medium with $k(\mathbf{r})=2 \pi / \lambda$. The simulation grid had a size of 1 by 200 pixels, with a resolu- 
tion of $\Delta x=\lambda / 4$. A source with unit amplitude was placed at the first pixel of the homogeneous medium. We used periodic boundary conditions in the vertical direction, and appended a 25- $\lambda$-thick absorbing layer (of type $N=4$, see Appendix 6.7.2) on both horizontal sides of the medium. The resulting structure of 1 by 400 pixels was zero-padded to a 1 by 512 pixel grid to increase the performance of the fast Fourier transforms. We simulated wave propagation through this medium. First using our modified Born approach, and then using PSTD with various values for $\Delta t$. For both methods, we chose the total number of iterations such that a distance of 100 wavelengths was traversed, i. e. the complete medium twice.

For each simulation, we calculated the relative error $E$ with respect to the analytic solution

$$
E \equiv \frac{\left\langle\left|\psi-\psi_{a}\right|^{2}\right\rangle}{\left\langle\left|\psi_{a}\right|^{2}\right\rangle},
$$

where $\psi_{a}$ is the exact analytic solution and $\langle\cdot\rangle$ denotes averaging over all pixels in the medium, excluding the boundaries. To calculate the $\psi_{a}$, we represent the single-pixel source as a sinc function, and convolve this source with the Green's function for a homogeneous medium. For simplicity, we only evaluate the convolution for $x>0$

$$
\begin{aligned}
\psi_{a}(x) & =\frac{\sin (\pi x / \Delta x)}{\pi x / \Delta x} * \frac{e^{i k|x|}}{-2 i k} \\
& =\frac{\Delta x}{4 k \pi} e^{-i k x}\left[2 i \pi+\operatorname{Ei}\left(i k^{-} x\right)-\operatorname{Ei}\left(i k^{+} x\right)\right]+\frac{\Delta x}{4 k \pi} e^{i k x}\left[\operatorname{Ei}\left(-i k^{-} x\right)-\operatorname{Ei}\left(-i k^{+} x\right)\right]
\end{aligned}
$$

with $k^{-} \equiv k-\pi / \Delta x, k^{+} \equiv k+\pi / \Delta x$, and Ei as the exponential integral function. The solution rapidly converges to $\Delta x \exp (i k x) /(-2 i k)$ with increasing distance from the source. Still, with the accuracy that our method achieves, the contribution of the exponential integrals $(0.06 \%$ of the total energy in the simulation) cannot be neglected.

The result of the simulations are shown in Fig. 6.2. The figure shows that our method achieves a relative error $E$ of less than $10^{-11}$ at an exceptionally low computational effort of only 0.5 iteration per propagated wavelength. For PSTD, we varied $\Delta t$ from the largest possible value allowed by the convergence criterion (see Table 6.1), decreasing the time step until the relative error stabilized. The results of PSTD for large time steps are completely off ( $>100 \%$ relative error). To achieve an accuracy of $1 \%$ over the full medium, at least 55 iterations per propagated wavelength are needed. The execution speed of both our method and PSTD are limited by the two $\mathrm{fft}_{2}$ 's per iteration, so the $x$-axis of Fig. 6.2 is directly proportional to simulation time.

This simple test shows that our method is two orders of magnitude faster, and at least nine orders of magnitude more accurate than a PSTD simulation that is designed to achieve a $1 \%$ accuracy. As can be seen in the figure, the accuracy of PSTD can be increased by increasing the number of steps per wave cycle. Even though the accuracy is proportional to $\Delta t^{4}$, it takes an extreme value of well over $10^{4}$ iterations per wave cycle before the accuracy of PSTD matches that of our method. The last data point in Fig. 6.2 took 1.5 days to calculate, compared to 0.3 seconds for our modified Born series.

The main source of error in PSTD simulations is an accumulating error in the phase of the propagating wave due to an error in the calculated wavevector [16]. Under some circumstances (e.g. near-paraxial wave propagation) this error may not be a cause of concern. 


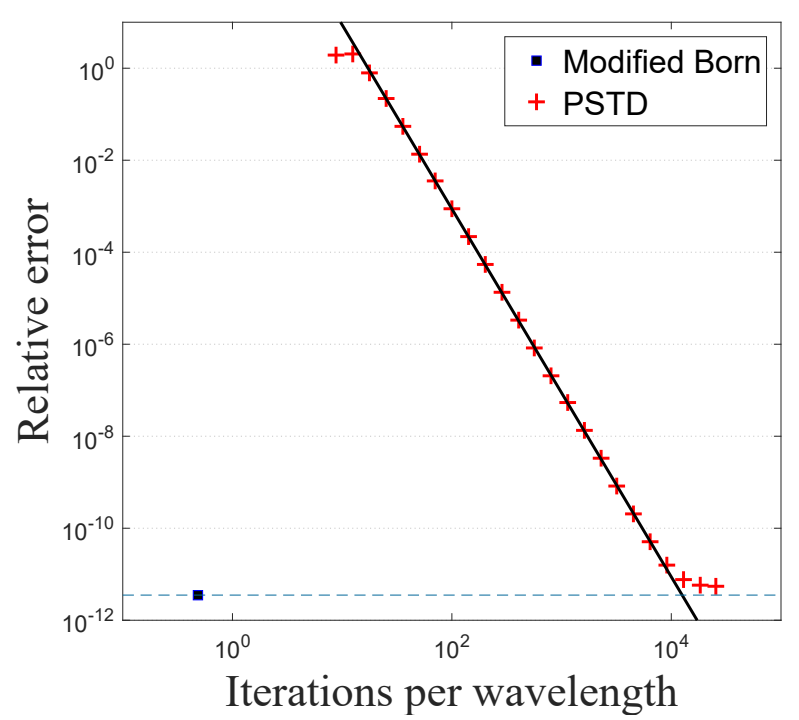

Figure 6.2: Relative error for wave propagation in a 1-D homogeneous medium as function of the number of iterations per wave cycle. Plus signs denote the errors for PSTD with varying time step $\Delta t$. The square denotes the error for our modified Born approach. The solid line represents the expected error of PSTD, which is proportional to $\Delta t^{4}$. The dashed line at $3.5 \cdot 10^{-12}$ corresponds to the accuracy achieved with our modified Born method.

However, in most interesting scenarios (such as resonators, scattering media, integrated optics devices, etc.) the phase needs to be calculated with a high accuracy in order to correctly simulate multi-path wave interference. Therefore, this simulation, where only 50 wave cycles were simulated, may even give an optimistic picture of the accuracy of PSTD. The relative error of $10^{-11}$ that is found for our method is caused by residual reflection and leakage of the absorbing boundaries, not by the algorithm itself. By choosing boundaries that are thicker and smoother, an error of less than $10^{-17}$ was reached. However, since such extreme accuracies are rarely required, we used thinner boundaries, resulting in a smaller medium and shorter run times.

\subsubsection{Disordered inhomogeneous medium}

In order to demonstrate the convergence of the modified Born approach for inhomogeneous media, we simulated wave propagation through a 2-dimensional inhomogeneous medium with a random complex potential. The relative scattering potential $k^{2}(\mathbf{r}) / k_{0}^{2}-1$ is normally distributed with mean $(1.30+0.05 i)^{2}$ and variance $(0.10+0.02 i)^{2}$. The distribution is lowpass filtered with a cut-off at $1.0 \lambda^{-1}$. The simulated medium has a size of 256 by 256 pixels with a resolution of $\Delta x=\min \{\lambda / 4\}$ in both spatial dimensions $x$ and $y$. Periodic boundaries were used and as a source term we used a point source in the center of medium at $x=y=0$.

First, we simulated wave propagation in the inhomogeneous medium using our method. The solution is presented in Fig. 6.3. Then, the numerical experiment was performed using PSTD with various numbers of iterations per wave cycle. Similar to in Eq. (6.25), we define 


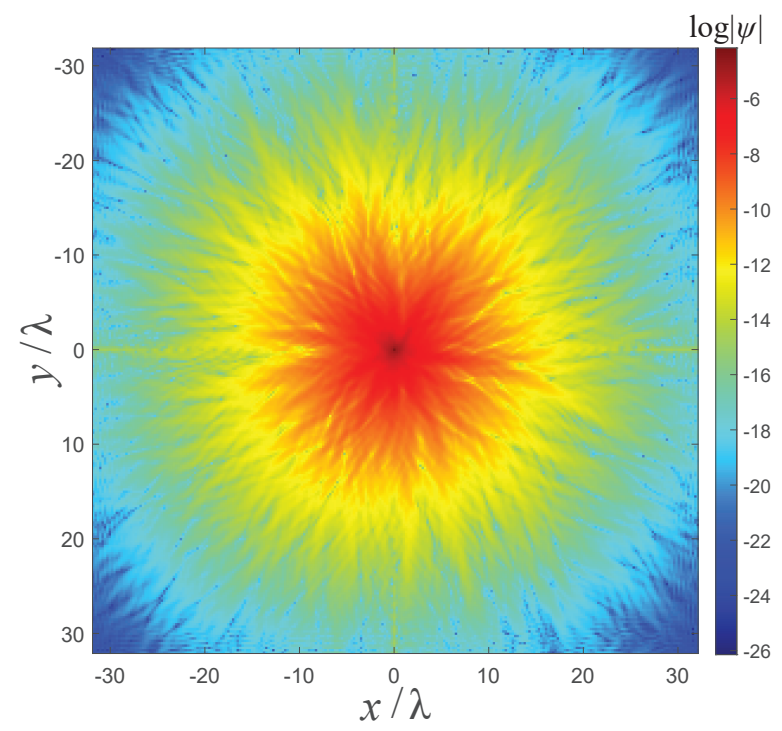

Figure 6.3: Numerical experiment simulating an oscillating point source at the center of an inhomogeneous medium with a random potential distribution. The steady-state solution is found using our modified Born approach.

a relative difference:

$$
E_{\text {diff }} \equiv \frac{\left\langle\left|\psi_{\text {PSTD }}-\psi_{\text {Born }}\right|^{2}\right\rangle}{\left\langle\left|\psi_{\text {Born }}\right|^{2}\right\rangle},
$$

where $\psi_{\mathrm{PSTD}}$ and $\psi_{\mathrm{B} \text { Brn }}$ are the solutions found using PSTD and our method respectively.

The resulting PSTD solution found using a large number of iterations per wave cycle indicates that the solution found by our method was identical to the solution found by PSTD in the limit $\Delta t \rightarrow 0$, to an accuracy of at least $10^{-13}$. One should note that the numerical experiment was completed in 8 seconds by the modified Born series method, whereas the PSTD method with $10^{4}$ iterations per wavelength required in order to reach $E_{\text {diff }}<10^{-13}$ took 20 hours to finish.

\subsubsection{Phase conjugation numerical experiments in adipose tissue}

In the third numerical experiment, we simulate a phase conjugation experiment inside a twodimensional adipose tissue sample of 725 by $725 \mu \mathrm{m}$ in size. Previously, media of such sizes were too time-consuming to simulate. For instance, simulating phase conjugation in a $240 \mu \mathrm{m}$ thick medium took one week [28], whereas our method took 15 minutes to simulate a $725 \mu \mathrm{m}$ thick medium.

We rewrite the wavenumber $k(\mathbf{r})=n(\mathbf{r}) 2 \pi / \lambda_{0}$, with $n(\mathbf{r})$ being the refractive index of the medium and $\lambda_{0}$ being the wavelength in vacuum. The refractive index distribution of the adipose tissue model is created using a gray scale microscopy image (Young et al. [27]). Based on the optical properties presented in the paper by Jacques [29], the refractive index of fatty cells and the extracellular fluids were estimated at 1.44 and 1.36 respectively. Given this range of refractive indices, the gray values in the microscopy image are linearly converted 


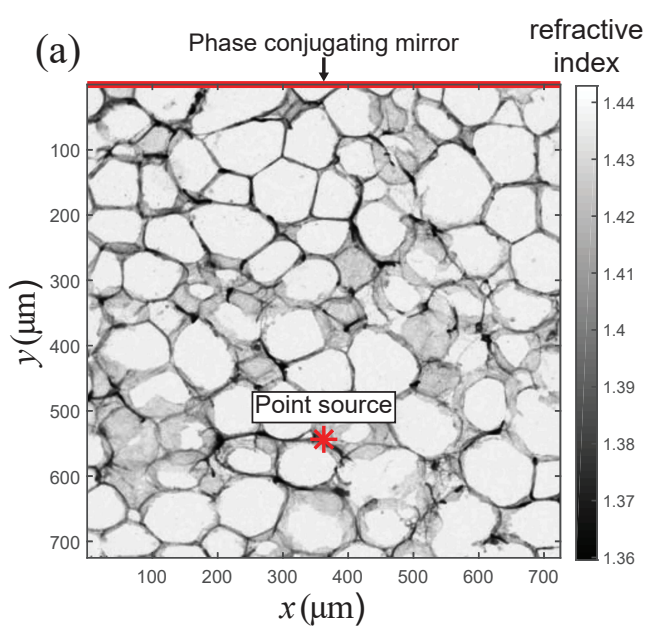

(b)

$\log |\psi|$

(c)

$\log |\psi|$
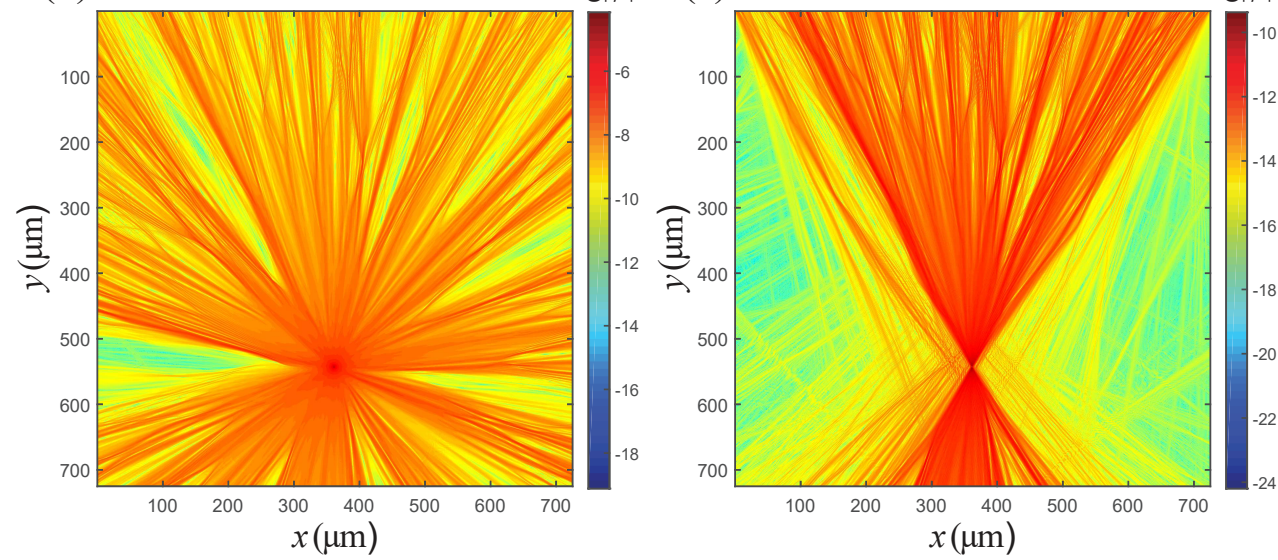

Figure 6.4: Numerical experiment simulating phase conjugation of light coming from a point source inside a two-dimensional adipose tissue model. (a) The refractive index distribution is based on a gray scale microscopy image of adipose tissue [27]. The red star marks the location of the point source and the line at the top boundary marks the location of the phase conjugating mirror. (b) The steady state solution of the recording phase, where light is propagated from a point source through the adipose tissue medium. (c) The steady state solution of the playback phase, where the field at the phase conjugating mirror is conjugated and propagated back into the medium. The simulation demonstrates that the light is being focused back at the original location of the point source. 


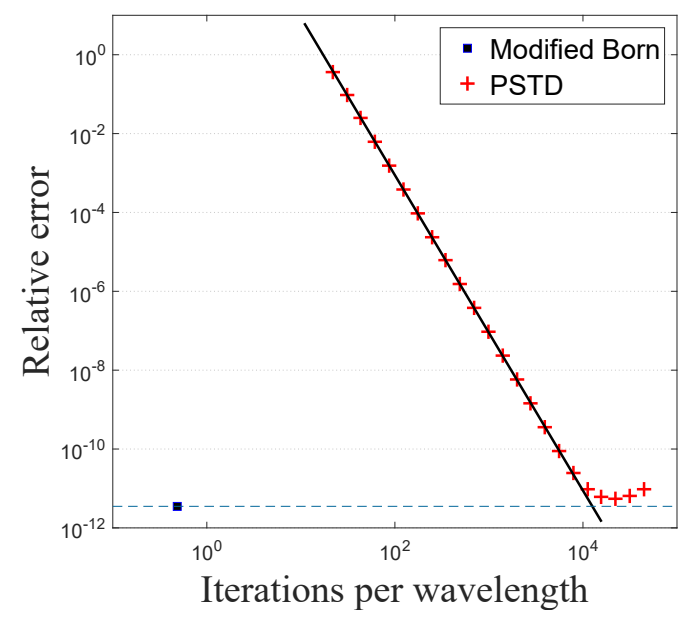

Figure 6.5: Relative error for a wave propagating in a 3-D homogeneous medium as a function of a number of iterations per wave cycle for our modified Born approach and PSTD with varying time-step. The solid line is proportional to $\Delta t^{4}$, with $\Delta t$ the PSTD time step. The dashed line at $3.5 \cdot 10^{-12}$ corresponds to the accuracy achieved with our modified Born method.

to a refractive index map shown in Fig. 6.4a. We chose $\lambda_{0}=1.0 \mu \mathrm{m}$ and we scaled the microscope image such that the pixel size $\Delta x=\lambda_{0} / 4$. The resulting medium size is 2900 by 2900 pixels, to which we added a $25-\lambda$-thick absorbing boundary layer and zero padded the medium to a size of 4096 by 4096 pixels. A point source is defined at at a depth of $y=48 \lambda$ inside the tissue model and a phase conjugating mirror is placed at the top boundary of the simulation grid.

A phase conjugation experiment consist of two phases: the recording phase and the digital playback phase. In the recording phase, we simulate the light propagation from a point source through the adipose tissue medium, which yields the steady-state solution as shown in Fig. 6.4b. Afterwards, we take the phase conjugate of the resulting field at the phase conjugating mirror at the top boundary of the simulation grid. This conjugated field is used as the source in the playback phase, which results in the steady-state solution shown in Fig. 6.4c. As can be seen in this figure, the light is still scattered as it propagates deeper inside the tissue, however, now it focuses at the location of the point source of the recording phase. Phase conjugation is an important technique for high-resolution imaging inside scattering media [30] and is one the many wave phenomena that can now be studied in large scattering structures using our modified Born series approach.

\subsubsection{Three-dimensional media}

Our method can easily be extended to solve the 3-dimensional Helmholtz equation. We simply replaced $\mathrm{fft}_{2}$ and ifft $_{2}$ in Eq. (6.20) by 3-dimensional fast Fourier transforms. To demonstrate the efficiency and accuracy for a 3-dimensional problem, we first simulated wave propagation in a homogeneous medium of $8 \times 8 \times 200$ pixels. This medium is equivalent to the medium used in Section 6.5.1, except for the extended size in the lateral dimensions. As 


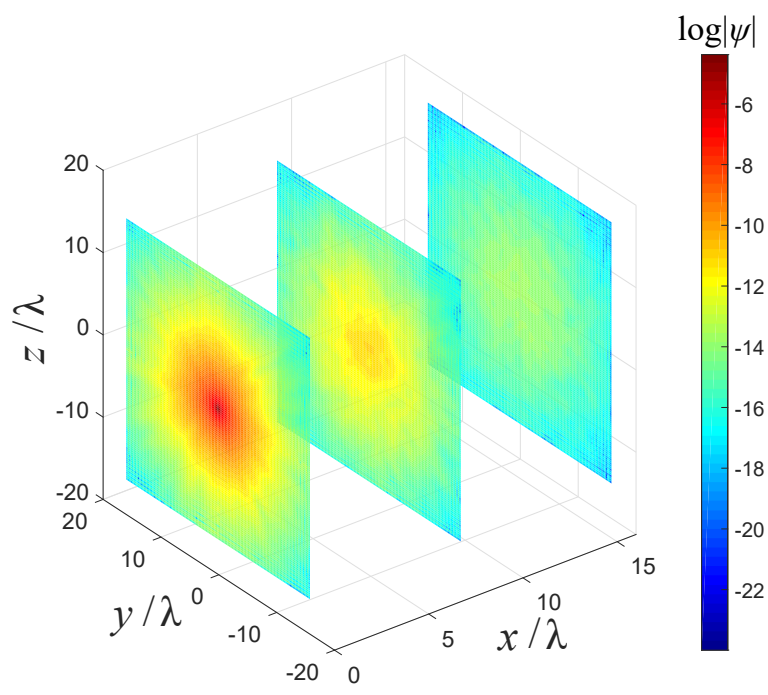

Figure 6.6: Simulation results for a point source at $x=y=z=0 \lambda$ in a 3-D disordered medium of $128 \times 128 \times 128$ pixels. The $3 \mathrm{D}$-solution is represented by three cross-sectional slices at $x=0 \lambda, x=8 \lambda$, and $x=16 \lambda$. The interference fringes at the boundaries of the slices are the result of the periodic boundary conditions.

can be seen in Fig. 6.5, the increase in speed and accuracy for the 3-D problem are identical to that of the 1-D problem (Fig. 6.2). In addition, it can be seen that the PSTD simulations become less accurate at very small time steps $\left(>10^{4}\right.$ iterations per wave cycle). When so many steps are used the rounding errors from the double precision floating point numbers start to accumulate, thereby limiting the accuracy of the result. Since the rounding errors accumulate with every iteration, further decreasing the time step only reduces the accuracy of PSTD.

Finally, we repeat the simulations of a disordered medium, this time in three spatial dimensions $x, y$ and $z$. We used the same parameters as in Section 6.5.2, with a medium size of $128 \times 128 \times 128$ pixels (equivalent to $32 \times 32 \times 32$ times the wavelength in vacuum), and placed the source at $x=y=z=0 \lambda$. Figure 6.6a-c show cross sections of the simulation results at $x=0 \lambda, x=8 \lambda$, and $x=16 \lambda$, respectively. Again, we compared the results of the modified Born series with PSTD and find that PSTD converges to the solution of our method in the limit $\Delta t \rightarrow 0$ to an accuracy of at least $10^{-9}$. The simulations took 14.6 seconds for our method, whereas the most accurate PSTD simulation using $10^{3}$ iterations per propagated wavelength took 19 hours.

\subsection{Conclusion}

In conclusion, we presented a fast iterative method for solving the Helmholtz equation. Our method is a modified version of the Born series. In contrast to the original Born series, our method converges for arbitrarily large structures with an arbitrarily high scattering potential. This guaranteed convergence is achieved by introducing the preconditioner $\gamma$ and the non- 
vanishing $\varepsilon$, which localizes the energy of the Green's function without affecting the solution of the Helmholtz equation.

Our method is several orders of magnitude faster in finding a steady-state solution to the inhomogeneous wave equation than the commonly used PSTD and FDTD methods, especially for media with a low scattering contrast. In addition, our method converges to the exact solution, whereas finite difference methods are, by definition, limited in accuracy.

We demonstrated our method for 1, 2, and 3-dimensional media. Extensions to scalar waves in higher dimensions are trivial. If a time-dependent solution is required, our modified Born series method could be applied once for each frequency of interest, and the timedependent solution could be calculated simply with a Fourier transform. Such an approach would still be more accurate, and potentially faster than PSTD, depending on the simulated bandwidth.

As with all grid-based numerical methods, the scattering potential has to be discretized, which imposes a limit on the spatial frequencies of the solution. This limitation will reduce the accuracy for media with very sharp interfaces. Furthermore, the speed of the modified Born series depends on the scattering contrast. Since most optical systems have refractive index typically in the range of 1 and 2, scattering contrast in optical systems is relatively small, which makes our method suitable for simulations of light propagation. However, in acoustic wave simulations, for instance, the scattering contrast can become much larger, drastically reducing the speed of our numerical method.

Future research may be directed towards finding combination of $\gamma$ and $\varepsilon$ for which the iterations converge more rapidly, or towards using more advanced iterative methods to speed up convergence. Additionally, due to the localized nature of our Green's function, a large medium may be split into separate domains to accommodate parallel processing or multidomain calculations.

\subsection{Appendices}

\subsubsection{Proof of convergence}

In this appendix, we will show that the series in Eq. (6.12) always converges for the choice of $\gamma$ and $\varepsilon$ as given by Eqs. (6.10) and (6.11). A sufficient condition for convergence is $\rho(M)<1$. We will first prove that $\rho(M) \leq 1$ and consider the eigenvalues with value 1 later. Recognizing that

$$
\frac{1}{|\mathbf{p}|^{2}-k_{0}^{2}-i \varepsilon}=\frac{i}{2 \varepsilon}\left(1-\frac{|\mathbf{p}|^{2}-k_{0}^{2}+i \varepsilon}{|\mathbf{p}|^{2}-k_{0}^{2}-i \varepsilon}\right),
$$

and substituting Eq. (6.10) we can write operator $M$ as

$$
\begin{aligned}
M & =\frac{-V}{2 \varepsilon^{2}}\left[1-F^{-1} \frac{|\mathbf{p}|^{2}-k_{0}^{2}+i \varepsilon}{|\mathbf{p}|^{2}-k_{0}^{2}-i \varepsilon} F\right] V-\frac{i V}{\varepsilon}+1, \\
& =\frac{1}{2 \varepsilon^{2}}\left[-V^{2}+V U V-2 i \varepsilon V+2 \varepsilon^{2}\right],
\end{aligned}
$$

where

$$
U \equiv F^{-1} \frac{|\mathbf{p}|^{2}-k_{0}^{2}+i \varepsilon}{|\mathbf{p}|^{2}-k_{0}^{2}-i \varepsilon} F
$$


is a unitary operator. In order to prove that $\rho(M) \leq 1$, it suffices to show that $|\langle x, M x\rangle| \leq\langle x, x\rangle$, for all $x$, where $\langle\cdot, \cdot\rangle$ is the inner product. We now use the Cauchy-Schwartz inequality to write $|\langle x, V U V x\rangle|=\left|\left\langle V^{\dagger} x, U V x\right\rangle\right| \leq \sqrt{\langle U V x, U V x\rangle} \sqrt{\left\langle V^{\dagger} x, V^{\dagger} x\right\rangle}=\langle V x, V x\rangle$, where we used the fact that $V$ is diagonal (and, hence $V^{\dagger} V=V V^{\dagger}$ ) in the final step. With this result, we can eliminate $U$, resulting in

$$
|\langle x, M x\rangle| \leq \frac{1}{2 \varepsilon^{2}}\left|\left\langle x,\left[2 \varepsilon^{2}-2 i \varepsilon V-V^{2}\right] x\right\rangle\right|+\frac{1}{2 \varepsilon^{2}}\langle V x, V x\rangle,
$$

To complete the proof, we now need to demonstrate that the right hand side of this equation is never larger than 1 . Since $V=V(\mathbf{r})$, we require

$$
\left|2 \varepsilon^{2}-2 i \varepsilon V(\mathbf{r})-V^{2}(\mathbf{r})\right|+|V(\mathbf{r})|^{2} \leq 2 \varepsilon^{2},
$$

for all $\mathbf{r}$. To show that this condition is always fulfilled, we define $\Delta \equiv V+i \varepsilon=k^{2}(\mathbf{r})-k_{0}^{2}$ and rewrite Eq. (6.34) as

$$
\left|\varepsilon^{2}-\Delta(\mathbf{r})^{2}\right|+|\Delta(\mathbf{r})-i \varepsilon|^{2} \leq 2 \varepsilon^{2}
$$

which can be written as

$$
\left.\left|\varepsilon^{2}-\right| \Delta(\mathbf{r})\right|^{2}-\left.2 i \Delta(\mathbf{r}) \operatorname{Im}\{\Delta(\mathbf{r})\}|+| \Delta(\mathbf{r})\right|^{2}+\varepsilon^{2}-2 \varepsilon \operatorname{Im}\{\Delta(\mathbf{r})\} \leq 2 \varepsilon^{2} .
$$

A slightly stricter criterion follows from triangle inequality

$$
\left.\left.\left|\varepsilon^{2}-\right| \Delta(\mathbf{r})\right|^{2}|+2| \Delta(\mathbf{r})|\operatorname{Im}\{\Delta(\mathbf{r})\}+| \Delta(\mathbf{r})\right|^{2}+\varepsilon^{2}-2 \varepsilon \operatorname{Im}\{\Delta(\mathbf{r})\} \leq 2 \varepsilon^{2},
$$

where we require that $\operatorname{Im}\{\Delta(\mathbf{r})\} \geq 0$, which means that the medium cannot have any gain. Since we have $\varepsilon \geq|\Delta(\mathbf{r})|$ from Eq. (6.11), the condition in Eq.(6.37) is always fulfilled, and therefore $\rho(M) \leq 1$.

Eigenvalues of 1 are only possible for an infinite non-absorbing medium. In this case, the solutions to the Helmholtz equation carry infinite total energy, which means that the solution cannot be found using our method. However, if there are absorbing boundaries, or even if there is a single a finite-size volume with non-zero absorption, there will be some points where the wave is absorbed. In this case the left hand side of Eq. (6.37) is strictly less than the right hand side, and convergence of our method is guaranteed.

\subsubsection{Boundary conditions}

In the implementation of our method, we use a fast Fourier transform to evaluate the convolution with the Green's function. As a result, the system has periodic boundary conditions by default. In order to prevent waves to 'wrap around' the boundaries, we implement absorbing boundary layers. A wide choice of boundary conditions is available for simulating scalar wave propagation in finite size systems (e.g. [31,32]). We choose to use a type of absorbing boundaries with the following properties: firstly, the layer is designed to have zero reflectivity for normal incidence. Secondly, the scattering potential $\left|k^{2}(\mathbf{r})-k_{0}^{2}\right|$ of the layer is bounded to a specified maximum value.

We design the absorbing layers by requiring that the wave has the following form

$$
\psi(x) \propto P_{N}(x) e^{i k_{0} x-\alpha x},
$$


with $P_{N}$ an N'th order polynomial inside the absorbing layer $(x>0)$ and equal to 1 for $x \leq 0$. Substituting this desired solution into Eq. (6.1) gives

$$
P_{N}^{\prime \prime}(x)+2\left(i k_{0}-\alpha\right) P_{N}^{\prime}(x)+\left[k^{2}(x)-k_{0}^{2}-2 i k_{0} \alpha+\alpha^{2}\right] P_{N}(x)=0 .
$$

The simplest solution is found for $N=1$ :

$$
\begin{gathered}
P_{1}(x>0)=1+\alpha x, \\
k^{2}(x)-k_{0}^{2}=\frac{\alpha^{2}\left(1-\alpha x+2 i k_{0} x\right)}{1+\alpha x} .
\end{gathered}
$$

Theoretically, this absorbing boundary has zero reflectivity. However, due to the discretization of the field, waves with a high spatial frequency cannot be represented. These frequency components are required to truthfully represent the waves at the transition from the medium to the absorbing boundary. When these components are missing, some residual reflectivity results. We found that this reflectivity can be reduced by smoothing the boundary. This can be achieved by imposing the constraint that the function $k^{2}(x)-k_{0}^{2}$ and its first $N-2$ derivatives vanish at $x=0$. A general solution for a polynomial of degree $N$ is given by

$$
\begin{gathered}
P_{N}(x>0)=\sum_{n=0}^{N} \frac{(\alpha x)^{n}}{n !} \\
k^{2}(x)-k_{0}^{2}=\frac{\alpha^{2}\left(N-\alpha x+2 i k_{0} x\right)(\alpha x)^{N-1}}{P_{N}(x) N !} .
\end{gathered}
$$

Choosing a higher value for $N$ results in a smoother boundary with a lower residual reflection. However, it also takes longer for the boundary to reach its maximum absorption coefficient of $\alpha$. In Eq. (6.42) it can be seen that $P_{N}(x)$ equals the first $N$ terms of the Taylor expansion of $\exp (\alpha x)$, thereby partially canceling the decay term $\exp (-\alpha x)$ close to the boundary.

The boundary potential saturates at a value of $k^{2}(x)-k_{0}^{2}=-\alpha^{2}+2 i k_{0} \alpha$ for $x \rightarrow \infty$, irrespective of $N$. Higher values of $\alpha$ result in stronger absorption, at the cost of increasing the scattering potential. Because of condition Eq. (6.11), a higher scattering potential results in a higher value of $\varepsilon$, which gives a lower pseudo-propagation speed (and hence, slower convergence of the method). Throughout this manuscript used layers with a thickness of 25 wavelengths, with $N=4$ and $\max \left|k^{2}(\mathbf{r})-k_{0}^{2}\right|=0.2$. These parameters gave a good balance between scattering potential, residual reflectivity, and residual transmission for our high-accuracy simulations.

\section{Acknowledgments}

This work was supported by the European Research Council (ERC-2016-StG-678919).

\section{Bibliography}

[1] M. C. W. van Rossum and T. M. Nieuwenhuizen, "Multiple scattering of classical waves: Microscopy, mesoscopy and diffusion”, Rev. Mod. Phys. 71, 313-371 (1999). 
[2] Y. A. Erlangga, "Advances in iterative methods and preconditioners for the Helmholtz equation”, Arch. Comput. Methods Eng. 15, 37-66 (2008).

[3] M. Nabavi, M. H. K. Siddiqui, and J. Dargahi, "A new 9-point sixth-order accurate compact finite-difference method for the Helmholtz equation”, J. Sound Vib. 307, 972982 (2007).

[4] J. M. Melenk and S. Sauter, "Convergence analysis for finite element discretizations of the Helmholtz equation with Dirichlet-to-Neumann boundary conditions”, Math. Comput. 79, 1871-1914 (2010).

[5] L. L. Thompson, “A review of finite-element methods for time-harmonic acoustics”, J. Acoust. Soc. Am. 119, 1315-1330 (2006).

[6] P. G. Martinsson, “A fast direct solver for a class of elliptic partial differential equations”, SIAM J. Sci. Comput. 38, 316-330 (2009).

[7] P. G. Schmitz and L. Ying, "A fast direct solver for elliptic problems on general meshes in 2d”, J. Comput. Phys. 231, 1314 - 1338 (2012).

[8] M. J. Gander, I. G. Graham, and E. A. Spence, "Applying GMRES to the Helmholtz equation with shifted Laplacian preconditioning: what is the largest shift for which wavenumber-independent convergence is guaranteed?", Numer. Math. 131, 567-614 (2015).

[9] C. C. Stolk, M. Ahmed, and S. K. Bhowmik, "A multigrid method for the Helmholtz equation with optimized coarse grid corrections", SIAM J. Sci. Comput. 36, 2819-2841 (2014).

[10] Y. A. Erlangga, C. W. Oosterlee, and C. Vuik, "A novel multigrid based preconditioner for heterogeneous Helmholtz problems”, SIAM J. Sci. Comput. 27, 1471-1492 (2006).

[11] C. C. Stolk, "A rapidly converging domain decomposition method for the Helmholtz equation”, J. Comput. Phys. 241, 240-252 (2013).

[12] D. Gordon and R. Gordon, "Robust and highly scalable parallel solution of the Helmholtz equation with large wave numbers", J. Comput. Appl. Math. 237, 182 196 (2013).

[13] O. G. Ernst and M. J. Gander, "Why it is difficult to solve Helmholtz problems with classical iterative methods", in Numerical analysis of multiscale problems, volume 83, 325-363 (Springer Berlin Heidelberg) (2012).

[14] A. Taflove and S. C. Hagness, Computational electrodynamics: The finite-difference time-domain method, Antennas and Propagation Library (Artech House) (1995).

[15] K. Yee, "Numerical solution of initial boundary value problems involving Maxwell's equations in isotropic media", IEEE Trans. Antennas Propag. 14, 302-307 (1966).

[16] Q. H. Liu, "The PSTD algorithm: A time-domain method requiring only two cells per wavelength", Microw. Opt. Technol. Lett. 15, 158-165 (1997). 
[17] J. L. Vay, I. Haber, and B. B. Godfrey, "A domain decomposition method for pseudospectral electromagnetic simulations of plasmas", J. Comput. Phys. 243, 260-268 (2013).

[18] S. H. Tseng, A. Taflove, D. Maitland, and V. Backman, "Pseudospectral time domain simulations of multiple light scattering in three-dimensional macroscopic random media”, Radio Sci. 41 (2006).

[19] B. Gallinet, J. Butet, and O. J. F. Martin, "Numerical methods for nanophotonics: standard problems and future challenges", Laser Photonics Rev. 9, 577-603 (2015).

[20] G. Beylkin, C. Kurcz, and L. Monzón, "Fast convolution with the free space Helmholtz green's function”, J. Comput. Phys. 228, 2770 - 2791 (2009).

[21] M. Wubs and A. Lagendijk, "Local optical density of states in finite crystals of plane scatterers", Phys. Rev. E 65 (2002).

[22] P. T. Kristensen, P. Lodahl, and J. Mørk, "Light propagation in finite-sized photonic crystals: multiple scattering using an electric field integral equation", J. Opt. Soc. Am. B 27, 228-237 (2010).

[23] O. J. F. Martin, A. Dereux, and C. Girard, "Iterative scheme for computing exactly the total field propagating in dielectric structures of arbitrary shape", J. Opt. Soc. Am. A 11, 1073-1080 (1994).

[24] R. E. Kleinman, G. F. Roach, and P. M. van den Berg, "Convergent Born series for large refractive indices”, J. Opt. Soc. Am. A 7, 890-897 (1990).

[25] S. Trattner, M. Feigin, H. Greenspan, and N. Sochen, "Validity criterion for the born approximation convergence in microscopy imaging”, J. Opt. Soc. Am. A 28, 665-666 (2011).

[26] C. Spa, P. Reche-López, and E. Hernández, "Numerical absorbing boundary conditions based on a damped wave equation for pseudospectral time-domain acoustic simulations", Sci. World J. 2014, 343-358 (2014).

[27] D. A. Young and K. L. Christman, "Injectable biomaterials for adipose tissue engineering”, Biomed. Mater. 29, 997-1003 (2012).

[28] S. H. Tseng, "PSTD simulation of optical phase conjugation of light propagating long optical paths", Opt. express 17, 5490-5495 (2009).

[29] S. L. Jacques, “Optical properties of biological tissues: A review”, Phys. Med. Biol. 58, R37-R61 (2013).

[30] R. Horstmeyer, H. Ruan, and C. Yang, "Guidestar-assisted wavefront-shaping methods for focusing light into biological tissue”, Nat. Photonics 9, 564-571 (2015).

[31] J. P. Berenger, "A perfectly matched layer for the absorption of electromagnetic waves", J. Comput. Phys. 114, 185-200 (1994). 
[32] Q. H. Liu, "The pseudospectral time-domain (PSTD) algorithm for acoustic waves in absorptive media", IEEE Trans. Ultrason. Ferroelectr. Freq. Control 45, 1044-1055 (1998). 


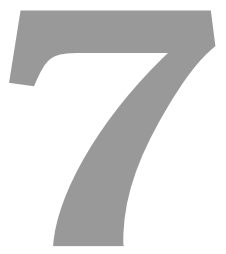

\section{Efficient boundary conditions for high-accuracy simulations of light propagation}

The modified Born series method is currently one of the most efficient methods available for simulating light scattering in large inhomogeneous media. However, to achieve high accuracy, the method requires thick gradually absorbing layers around the simulation domain. Here, we introduce new boundary conditions, combining an anti-cyclic convolution with an anti-reflection boundary layer. Our new boundary conditions minimize the wrap-around and reflection artefacts originating from the edges of the simulation domain, while also greatly reducing the memory requirements of the method. Our Matlab implementation is available on GitHub.

\subsection{Introduction}

Understanding how light propagates in structured media is a fundamental problem in many fields of optics. However, the analytical solution to the Maxwell equations can often only be found for simple systems, e.g. a Mie sphere [1]. For more complex problems, the solution has to be computed using numerical methods such as finite difference time domain [2-4], finite element [5] and psuedospectral time domain [6]. Although widely used in nanophotonics [7], these methods are too computationally demanding for large (three-dimensional) structures. In forward-scattering media, light propagation can be simulated using the beam propagation method $[8,9]$, where a scattering structure is modeled as a stack of infinitely thin phase plates. This method is commonly used to study light scattering in deep tissue microscopy [10-13]. While efficient and easy to implement, the beam propagation method is 
limited to forward scattering media, as paraxial light propagation is assumed and backscattered light is neglected.

A completely different category of numerical methods involve computing the Born series [14-16]. Recently, we developed a new modified Born series (MBS) method to solve the scalar wave equation [17]. We proved that our modifications guarantee convergence to the analytical solution for any inhomogeneous medium. We demonstrated that the MBS method is orders of magnitude faster and more accurate than state-of-the-art numerical techniques. Our method was later generalized for vector fields [18] and anisotropic media [19].

Ideally when simulating an open system, a light wave should vanish as it exits the simulation domain. To mimic such an open system, MBS, like many other numerical methods [20], requires padding of the domain with an absorbing boundary layer. For computational efficiency, this layer should be as thin as possible. However, if the layer does not absorb enough energy, a wave leaking through the absorbing boundary layer will re-enter the simulation domain from the opposite side (see Fig. 7.1a). This wrap-around effect is caused by the fast convolutions at the heart of the MBS algorithm. On the other hand, if the absorbing layer cuts off the electric field too abruptly, reflection artefacts arise, as shown in Fig. 7.1b. Until now, a thick gradually absorbing boundary layer was needed to achieve the maximum accuracy, increasing the computational costs and memory requirements of the algorithm. This problem becomes even more pressing when simulating light propagation in three-dimensional structures.

Here, we introduce a new class of boundary conditions for the MBS method, greatly reducing the memory requirements of the method, while suppressing the wrap-around errors and reflection artefacts (see Fig. 7.1c). The key novelty of our approach is to use left and right circular convolutions (first introduced by Radhakrishnan et al. [21]), and alternate them in a pipelined fashion. This way, wrap-around artefacts are cancelled exactly without affecting the computation time. The anti-cyclic convolution method is combined with a thin antireflection boundary layer. The performance of our new boundary conditions is evaluated in a three-dimensional test simulation.

\subsection{Theory}

\subsubsection{Modified Born series}

Here, we use the modified Born series method to solve the Maxwell equations for a given source $S$ with a wavenumber $k_{0}$ in a medium with refractive index distribution $n(\mathbf{r})$ [18]. The standard Born series is known to diverge for large inhomogeneous structures [14]. Therefore, in previous work, we made modifications to the Born series to guarantee convergence without affecting the solution [17]. The electric field $E$ can be obtained from the modified Born series, which is given by:

$$
\psi=[1+M+M M+M M M+\ldots] \gamma G S,
$$

with $M \equiv-i \varepsilon \gamma G \gamma-\gamma+1$. Here, $\varepsilon$ is a damping factor and $\gamma \equiv i\left[n(\mathbf{r})^{2}-1\right] k_{0}^{2} / \varepsilon$ is the medium operator. The operator $G$ represents the convolution with the Green's function. In the MBS algorithm, we iteratively compute the terms in Eq. (7.1) until the desired accuracy is reached. Starting with $\hat{\psi}_{1}=\gamma G S$, the difference field computed in $(j+1)$ th iteration is given by

$$
\hat{\psi}_{j+1}=M \hat{\psi}_{j} .
$$



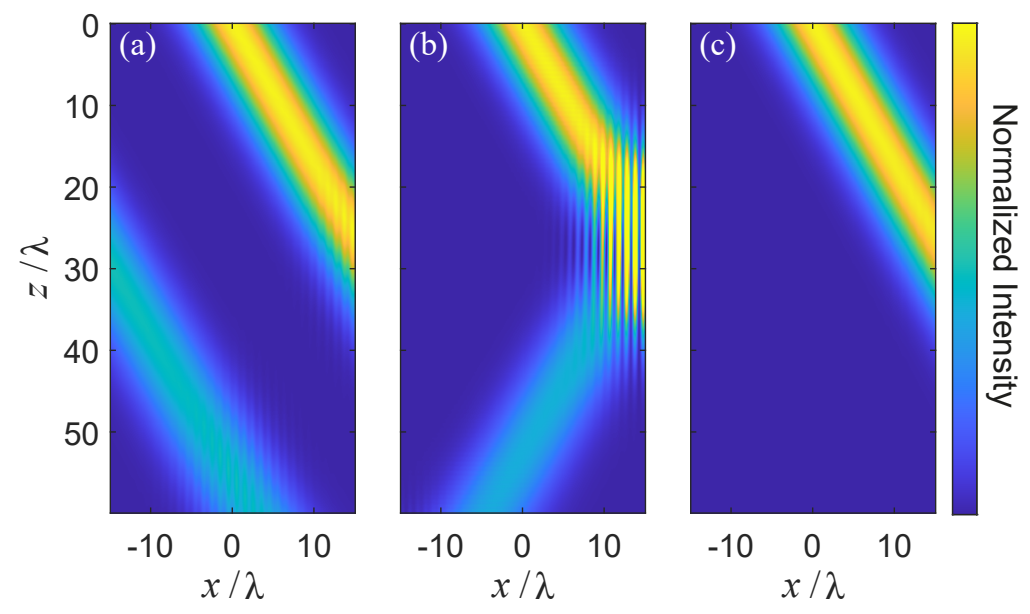

Figure 7.1: Potential errors in light simulations introduced by the boundary layers. (a) A simulation using thin weakly absorbing boundaries, where the light leaks through and re-enters through the opposite side. (b) A simulation using thin strongly absorbing boundaries, introducing strong reflection artefacts. (c) Simulation results obtained using the new boundary conditions without the wrap-around and reflection artefacts.

The solution, $E$, is obtained from accumulation of all computed difference fields $\hat{\psi}$.

With every iteration, the operator $M$ is applied to the difference field of the previous iteration. In $M$, the operator $\gamma$ applies the scattering introduced by the medium, whereas the operator $G$ 'propagates' the field through the medium.

For performance reasons, the convolution with the Green's function is implemented as a fast-convolution, with $G \equiv \mathscr{F}^{-1} \tilde{g}(\mathbf{k}) \mathscr{F}$. Here, $\mathscr{F}$ and $\mathscr{F}^{-1}$ represent the forward and inverse Fourier transforms and $\tilde{g}(\mathbf{k})$ is the Green's function in Fourier coordinates k. Since the simulation domain is finite, $G$ is a cyclic convolution, resulting in the wrap-around artefacts as can be seen in Fig. 7.1a. In the next section, we will discuss how these wrap-around errors can be removed.

\subsubsection{Anti-cyclic convolution}

Here, we describe an anti-cyclic convolution method based on the work of Radhakrishnan et al. [21]. This method is used to eliminate the wrap-around errors introduced by applying operator $G$ to a field $\psi$. To simplify the notation, we start by considering a one dimensional problem with spatial coordinates $x \in(-L / 2, L / 2)$.

First, the modified Fourier transforms

$$
\overleftarrow{\mathscr{F}} E \equiv \int_{-L / 2}^{L / 2} E(x) e^{-i(\mathrm{k}+\Delta \mathrm{k} / 4) x} d x
$$

and

$$
\overrightarrow{\mathscr{F}} E \equiv \int_{-L / 2}^{L / 2} E(x) e^{-i(\mathrm{k}-\Delta \mathrm{k} / 4) x} d x
$$



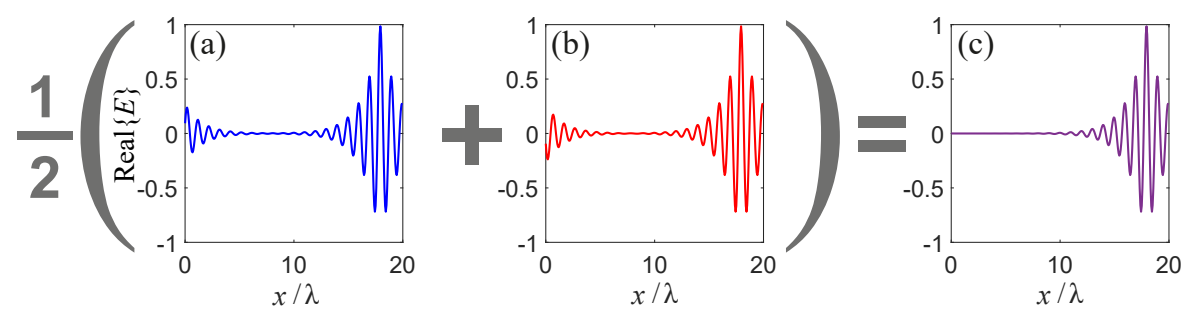

Figure 7.2: One-dimensional demonstration of the anti-cyclic convolution. The Green's function is convolved with a point source (located at $x=18 \lambda$ ). The results obtained using (a) the left circular convolution and (b) the right circular convolution. Wrap-around artefacts can be observed at the left side of the simulation grid in both these figures. (c) Results obtained from the anti-cyclic convolution, completely canceling the wrap-around artefacts.

are introduced, where $\Delta \mathrm{k} \equiv 2 \pi / L$ is the grid spacing in Fourier space. Compared to a standard Fourier transform, the modified operators $\overleftarrow{\mathscr{F}}$ and $\overrightarrow{\mathscr{F}}$ modulate the field with a linear phase ramp from $-\pi / 4$ to $\pi / 4$, or vice versa, before transforming the field to Fourier coordinates. Applying these phase ramps in real-space is equivalent to a translation of the field in k-space of $\pm \Delta \mathrm{k} / 4$. These operations are reversed by the modified inverse modified Fourier transform operators $\overleftarrow{\mathscr{F}}^{-1}$ and $\overrightarrow{\mathscr{F}}^{-1}$, that first perform an inverse Fourier transform and then remove the phase ramp.

Next, we define the modified versions of the Green's function operator using the modified Fourier transforms. The operator $G$ is replaced with the left-circular and right-circular Green's function operators, which are given by

$$
\overleftarrow{G} \equiv \overleftarrow{\mathscr{F}}^{-1} \tilde{g}(\mathrm{k}+\Delta \mathrm{k} / 4) \overleftarrow{\mathscr{F}} \text { and } \vec{G} \equiv \overrightarrow{\mathscr{F}}^{-1} \tilde{g}(\mathrm{k}-\Delta \mathrm{k} / 4) \overrightarrow{\mathscr{F}}
$$

respectively. In words, $\psi$ is transformed to a shifted k-space grid, and then multiplied with the shifted Green's function in k-space. Afterwards, the field is transformed back to real-space and the phase ramp is reversed.

Note, that the phase ramp $\pm \Delta k / 4$ has a discontinuity $\mp \pi / 2$ when wrapping around the edge of the domain. For the field that remains inside the simulation domain, this discontinuity has no effect: adding and later removing the phase ramp has no net effect and the operation is exactly equivalent to performing a fast convolution. For the part of the convolved field that leaked through the boundary, however, this discontinuity makes that the wrapped component has a phase shift $\pm \pi / 2$ compared to the non-wrapped component. Since the wrap-around errors introduced by the left-circular and right-circular convolutions have opposite signs, we can remove these errors by averaging the results of $\overleftarrow{G}$ and $\vec{G}$. Now we can substitute the Green's function operator $G \rightarrow(\overleftarrow{G}+\vec{G}) / 2$, arriving at the convolution method as described in Ref. [21]. We will refer to this method as the anti-cyclic convolution (ACC).

In Fig. 7.2, the anti-cyclic convolution is demonstrated in a simple example, where the Green's function is convolved with a point source. The results of $\overleftarrow{G}$ (see Fig. 7.2a) and $\vec{G}$ (see Fig. 7.2b) are averaged to obtain the anti-cyclic convolution results. As can be seen in Fig. 7.2c, the wrap-around errors are completely canceled by the ACC.

We only considered a one-dimensional problem here, but the anti-cyclic convolution can easily be generalized to higher dimensional problems [22]. However, all combinations of 
the left and right circular convolutions have to be considered in all dimensions, increasing the computation complexity by a factor of 4 and 8 for two and three-dimensional problems, respectively. Because of these extra computational costs, the applicability of the anti-cyclic convolution has been limited. In the next section, we show that in an iterative algorithm, such as the MBS algorithm, the different circular convolutions can be efficiently computed in a pipelined fashion.

\subsection{Implementation}

\subsubsection{Pipelined MBS algorithm}

We will now discuss how the anti-cyclic convolution is efficiently incorporated into the MBS method. We replace the operator $M$ with the left-circular and right-circular operators: $\overleftarrow{M} \equiv-i \varepsilon \gamma \overleftarrow{G} \gamma-\gamma+1$ and $\vec{M} \equiv-i \varepsilon \gamma \vec{G} \gamma-\gamma+1$. Directly implementing the anti-cyclic convolution into the MBS method would require us to apply both $\overleftarrow{M}$ and $\vec{M}$ in every iteration, which would double the computation time.

Instead, in our approach we alternate between the left-circular and right-circular operators.

$$
\hat{\psi}_{j+1}= \begin{cases}\overleftarrow{M} \hat{\psi}_{j} & \text { if } \mathrm{j} \text { is odd } \\ \vec{M} \hat{\psi}_{j} & \text { if } \mathrm{j} \text { is even }\end{cases}
$$

Every odd iteration uses $\overleftarrow{M}$, causing wrap-around artefacts to occur with a pre-factor $+i$. And every even iteration uses $\overleftarrow{M}$, causing the wrap-around artefacts to occur with a pre-factor $-i$. The idea is now to add the source term both in the first iteration and in the second iteration: $\hat{\psi}_{1}=\gamma \vec{G} S / 2$, and $\hat{\psi}_{2}=\overleftarrow{M} \hat{\psi}_{1}+\gamma \overleftarrow{G} S / 2$. This way we effectively compute two sequences simultaneously

$$
\begin{aligned}
\psi= & \frac{1}{2}(1+\overleftarrow{M}+\vec{M} \overleftarrow{M}+\overleftarrow{M} \vec{M} \overleftarrow{M}+\ldots) \gamma \vec{G} S+ \\
& \frac{1}{2}(1+\vec{M}+\overleftarrow{M} \vec{M}+\vec{M} \overleftarrow{M} \vec{M}+\ldots) \gamma \overleftarrow{G} S
\end{aligned}
$$

The two sequences differ only in that the left- and right-circular operators are swapped. Consequently, they give identical results except that the sign of the wrap-around artefacts is reversed. Therefore, when summing these sequences, the artefacts cancel exactly.

The second sequence in Eq. (7.8) is one iteration behind. This means that each wrap around artefacts is compensated for directly in the next iteration. Because of this 'pipelining", we can perform the anti-cyclic convolutions without having to perform any extra evaluations of the operator $M$. A similar algorithm is used when performing three-dimensional simulations, where we instead alternate between 8 different circular versions of the $M$ operator.

Our efficient algorithm can only correct for waves that wrap around the boundaries an odd number of times. Terms that correspond to passing the boundary twice (or any other even number of times) are not cancelled and return to the simulation domain with a $\pi$ or 0 phase shift depending on number of iterations between the passes. Fortunately, as becomes clear from the simulation results, these terms have a negligible contribution to the error. This is because they pass the absorbing boundary twice, and because the terms do not add constructively (since the phase is 0 for some terms and $\pi$ for the other). 


\subsubsection{Anti-reflection boundary layer}

The new algorithm suppresses the wrap-around artefacts that originate from the fast convolutions. However, for an accurate simulation, thin boundary layers are still required. Our pipelined anti-cyclic method introduces sharp transitions in the field exactly at the boundary causing reflections. The absorbing anti-reflection layer serves to minimize these reflections.

We design the boundary layers to have the maximum amount of absorption that can stably be simulated without increasing the damping factor $\varepsilon$. This maximum amount of absorption corresponds to $\gamma=0$ exactly. For simplicity, we implement the anti-reflection layer as a window function $B$ with which we multiply $\gamma$. B should be unity inside the simulation domain, as we do not want to affect the simulated structure, and it should vanish at the edge. Furthermore, $B$ should be smooth to minimize the amount of reflection caused by the boundary layer. Finally, $B$ should be real-valued with values between 0 and 1 to guarantee convergence of the MBS under all circumstances.

We design $B$ for a three-dimensional simulation of an open system, where $\gamma$ is padded in all directions with boundary layers, each with a width of $N_{B}$ grid points. Since the boundaries are identical in all direction, we will only consider the window function along the $z$-axis.

First we realize that the multiplication with the electric field in real-space is equivalent to a convolution in k-space. For an incident wave $E \propto \exp (i \mathbf{k} \cdot \mathbf{r})$, the reflection coefficient is determined by strongly this convolution couples the incident $\left(+k_{z}\right)$ and reflected $\left(-k_{z}\right)$ fields. In other words, the reflection coefficient is given by $\tilde{B}\left(k_{z}=-2 k_{0}\right)$, with $\tilde{B}\left(k_{z}\right)$ the Fourier transform of $B$.

To find the optimal window function that minimizes the reflection artefacts, we define a cost function

$$
C=\int_{-k_{0}}^{k_{0}} w\left(k_{z}\right)\left|\tilde{B}\left(2 k_{z}\right)\right|^{2} d k_{z},
$$

where $w$ is a weighting function. We take $w\left(k_{z}\right)=k_{z}^{2}$, meaning that reflections at an angle close to normal incidence are weighted more than the reflections at grazing incidence. Under this constraint, we find that $C$ is minimized by

$$
B\left(z_{b}\right)=\frac{\left|z_{b}\right|-0.21}{N_{b}+0.66}
$$

Here, $z_{b}$ are the grid indices at the boundary layer ranging from $-N_{b}$ to -1 and from 1 to $N_{b}$ at the top and bottom boundaries, respectively. The same window function is used along the $x$ and $y$ axes. Finally, the optimized boundary layer is then obtained by multiplying the padded $\gamma$ with this window function. We will refer to this new boundary type as the anti-reflection boundary layer (ARL). Here, we mainly considered small angle scattering from a structure, but the weighting function $w$ can be adjusted for other simulation geometries. The Matlab code used for the filter optimization can be found on GitHub [23].

\subsection{Performance test}

In this section, we compare the performance of MBS with the anti-cyclic convolution method (see Eq. (7.8)), and MBS with the conventional fast convolution method. These methods were tested using the new anti-reflection boundary layer and the polynomial boundary layer (PBL) 

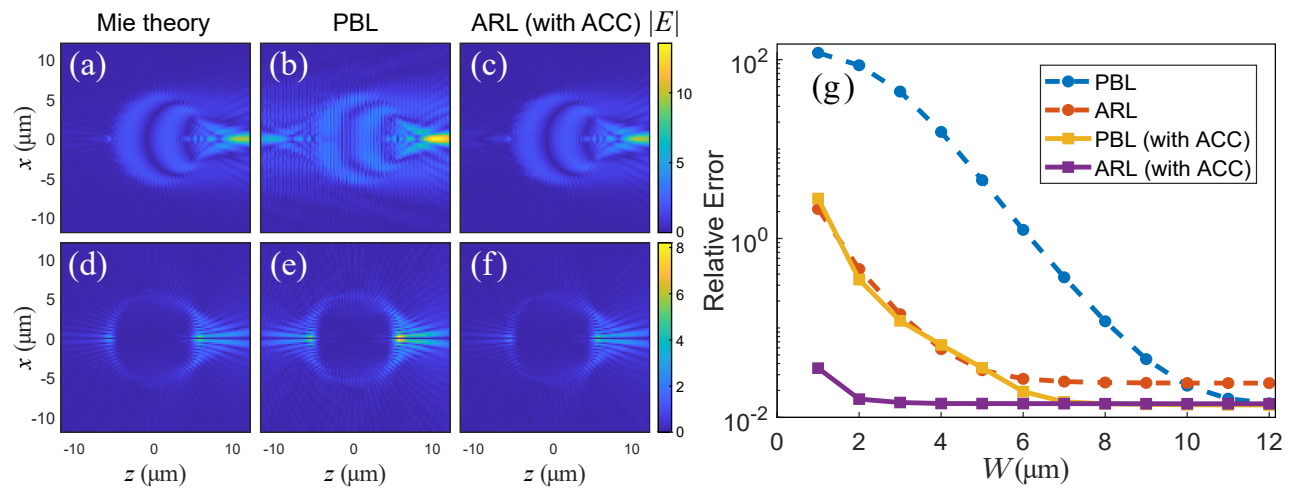

Figure 7.3: Three-dimensional Mie sphere simulation results. (a)-(f) Examples of the scattered field cross sections, taken at $y=0$ with $W=6 \mu \mathrm{m}$. (a)-(c) the x-component and, (d)-(f), the z-component of the scattered vector field amplitude. (a,d) Analytical solution obtained from Mie theory. (b,e) Simulation results using the polynomial boundary layer without ACC. (c,f) Simulation results using the anti-reflection boundary layer with ACC. (g) The relative error of the simulations for different boundary conditions and for varying boundary widths.

as implemented in our previous work [17]. The PBL was designed to give a good balance between suppressing the reflection and wrap-around artefacts at normal incidence.

To assess the performance of these different boundary conditions, we simulated the propagation of light in a Mie sphere with a diameter of $12 \mu \mathrm{m}$. We use refractive indices of 1.2 and 1 for the sphere and the background medium, respectively. The total simulation grid spanned over a volume of $24 \times 24 \times 24 \mu^{3}$ with a grid spacing of $0.20 \mu \mathrm{m}$. As the source, we use a linearly-polarized apodized plane wave with $\lambda=1 \mu \mathrm{m}$. The source is placed at $z=-12 \mu \mathrm{m}$, and is polarized in the $x$-direction. The scattered vector field, $\psi_{s}$, obtained from the simulation results, is compared to the results $E_{a}$ from Mie theory (generated with code from Ref. [24]). Boundary layers with a width of $W$ are added to all sides of the simulation volume. During the simulations, $W$ is varied from 1 to $12 \mu \mathrm{m}$. The accuracy of the simulation results is quantified by the relative error, given by: $\left\langle\left|E_{s}-E_{a}\right|^{2}\right\rangle /\left\langle\left|E_{a}\right|^{2}\right\rangle$. Here, $\langle\cdot\rangle$ denotes the averaging over all vector components and all grid points in the medium, excluding the boundary layers.

In Fig. 7.3, the results of the Mie sphere simulations are shown. As an example, cross sections of the scattered vector field amplitude are shown in Fig. 7.3a-f. The $x$-component and the $z$-component of the obtained fields are presented in Fig. 7.3a-c and Fig. 7.3d-f, respectively. These figures contain the analytical solution (Fig. 7.3a and d), the simulation results using the PBL without ACC (Fig. 7.3b and e), and the simulation result using the ARL with ACC (Fig. 7.3c and f). Finally, in Fig. 7.3g, the relative error of the simulation results are plotted against $W$. The blue and orange circles denote the simulation results obtained using the PBL and the ARL, respectively. The results obtained using the anti-cyclic convolution are denoted by the yellow and the purple squares for the PBL and ARL.

In the PBL simulation results, the plane wave source is not completely absorbed at the boundaries and wraps around the simulation domain. This leaked wave then interferes with the wave that scatters off the Mie sphere, resulting in a fringe pattern as can be seen in 
Fig. 7.3b. In Fig. 7.3e, these fringes are not observed in the $z$-component of the field, as the source was not polarized in that direction. However, in this figure, a wrap-around artefact can still be seen at the left side. In the ARL simulation results with the anti-cyclic convolution method, in Fig. 7.3c and f, the wrap-around artefact have been successfully removed.

In Fig. 7.3g, we can observe a decrease in the relative error with the increase of the boundary width for all boundary conditions. When considering the PBL simulations with $W=2 \mu \mathrm{m}$, the results obtained using the ACC method are almost up to 3 orders of magnitude more accurate than the simulations without ACC. Compared to these result, the introduction of the anti-reflection boundary adds another order of magnitude in accuracy. Using this ARL with ACC, the maximum achievable accuracy (a relative error of 0.015) is obtained using a boundary width of only $2 \mu \mathrm{m}$. In contrast, the PBL without ACC requires $W$ to be $12 \mu \mathrm{m}$ to reach the same accuracy. This difference leads to reduction of the total required simulation size from $(48 \mu \mathrm{m})^{3}$ to $(26 \mu \mathrm{m})^{3}$ for this three-dimensional simulation. Here, the maximum accuracy is limited by the discretization of the Mie sphere, and can be further improved by performing the simulation on a finer grid.

\subsection{Conclusion}

We have introduced a new class of boundary conditions for the modified Born series method, increasing both the accuracy and the memory efficiency of the method. First, we replaced the fast convolution operation in the algorithm with an anti-cyclic convolution. The additional steps in this convolution method were efficiently pipelined, such that the wrap-around artefacts can be removed without any extra computational costs. Secondly, we designed a new boundary layer which minimizes the reflection artefacts introduced at the edge of the simulation domain. In comparison with the previously implemented boundary conditions, we found that our new boundary conditions are up to 4 orders of magnitude more accurate. When simulating at the highest accuracy, the memory consumption of the MBS method was reduced by a total of $84 \%$ in our three-dimensional test simulation. We believe that our new boundary conditions could improve the accuracy and memory efficiency of other iterative methods with a fixed convolution kernel, such as the psuedo-spectral time domain method [6]. The Matlab code of our modified Born series method is available on GitHub [23].

\section{Bibliography}

[1] C. F. Bohren and D. R. Huffman, Absorption and scattering of light by small particles (Wiley-VCH) (1998).

[2] K. Yee, "Numerical solution of initial boundary value problems involving Maxwell's equations in isotropic media", IEEE Trans. Antennas Propag. 14, 302-307 (1966).

[3] A. Taflove and S. C. Hagness, Computational electrodynamics: The finite-difference time-domain method, Antennas and Propagation Library (Artech House) (1995).

[4] A. F. Oskooi, D. Roundy, M. Ibanescu, P. Bermel, J. D. Joannopoulos, and S. G. Johnson, "Meep: A flexible free-software package for electromagnetic simulations by the FDTD method", Comput. Phys. Commun. 181, 687-702 (2010). 
[5] P. Monk, Finite element methods for Maxwell's equations (Clarendon Press) (2003).

[6] Q. H. Liu, "The PSTD algorithm: A time-domain method requiring only two cells per wavelength”, Microw. Opt. Technol. Lett. 15, 158-165 (1997).

[7] B. Gallinet, J. Butet, and O. J. F. Martin, "Numerical methods for nanophotonics: standard problems and future challenges", Laser Photonics Rev. 9, 577-603 (2015).

[8] J. W. Goodman, Introduction to Fourier Optics, McGraw-Hill physical and quantum electronics series (W. H. Freeman) (2005).

[9] J. Van Roey, J. Van Der Donk, and P. E. Lagasse, "Beam-propagation method : analysis and assessment", J. Opt. Soc. Am. 71, 803-810 (1981).

[10] A. K. Glaser, Y. Chen, and J. T. C. Liu, "Fractal propagation method enables realistic optical microscopy simulations in biological tissues", Optica 3, 861-869 (2016).

[11] M. Weigert, K. Subramanian, S. T. Bundschuh, E. W. Myers, and M. Kreysing, "Biobeam-multiplexed wave-optical simulations of light-sheet microscopy", PLOS Comput. Biol. 14, 1-11 (2018).

[12] J. Yang, J. Li, S. He, and L. V. Wang, “Angular-spectrum modeling of focusing light inside scattering media by optical phase conjugation”, Optica 6, 250-256 (2019).

[13] X. Cheng, Y. Li, J. Mertz, S. Sakadžić, A. Devor, D. A. Boas, and L. Tian, "Development of a beam propagation method to simulate the point spread function degradation in scattering media”, Opt. Lett. 44, 4989-4992 (2019).

[14] R. E. Kleinman, G. F. Roach, and P. M. Van Den Berg, "Convergent Born series for large refractive indices”, J. Opt. Soc. Am A 7, 890-897 (1990).

[15] O. Pankratov, D. Avdeev, and A. Kuvshinov, "Electromagnetic field scattering in a homogeneous Earth: a solution to the forward problem", Phys. Solid Earth 31, 201-209 (1995).

[16] M. Zhdanov and S. Fang, "Quasi-linear series in 3-D electromagnetic modeling”, Radio Sci. 32, 462-465 (1997).

[17] G. Osnabrugge, S. Leedumrongwatthanakun, and I. M. Vellekoop, “A convergent Born series for solving the inhomogeneous Helmholtz equation in arbitrarily large media", J. Comput. Phys. 322, 113-124 (2016).

[18] B. Krüger, T. Brenner, and A. Kienle, "Solution of the inhomogeneous Maxwell's equations using a Born series”, Opt. Express 25, 25165-25182 (2017).

[19] T. Vettenburg, S. A. R. Horsley, and J. Bertolotti, "Calculating coherent light-wave propagation in large heterogeneous media”, Opt. Express 27, 11946-11967 (2019).

[20] J. P. Berenger, "A perfectly matched layer for the absorption of electromagnetic waves", J. Comput. Phys. 114, 185-200 (1994). 
[21] C. Radhakrishnan and K. Jenkins, "Modified discrete Fourier transforms for fast convolution and adaptive filtering", in ISCAS 2010 - 2010 IEEE International Symposium on Circuits and Systems: Nano-Bio Circuit Fabrics and Systems, 1611 - 1614 (2010).

[22] C. Radhakrishnan and W. K. Jenkins, "The 2-D modulated discrete fourier transform for 2-D fast convolution and digital filtering”, in 2011 IEEE International Symposium of Circuits and Systems (ISCAS), 1508-1511 (2011).

[23] G. Osnabrugge and I. M. Vellekoop, WaveSim (2016), https://github.com/ gosnabrugge/wavesim.

[24] J.-P. Schäfer, MatScat (2012), http://www.mathworks.com/matlabcentral/ fileexchange/36831-matscat. 


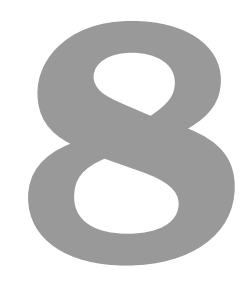

\section{Summary and outlook}

\section{Summary}

In microscopy, light scattering sets the limit for the maximum imaging depth inside biological tissue. In this thesis, we investigated how this fundamental limit can be broken by means of wavefront shaping (WFS), a powerful tool that provides control over the light inside scattering media. We studied the propagation of light in forward scattering media, and we developed new wavefront shaping techniques to exploit scattered light for deep tissue imaging.

With wavefront shaping, scattered light can be focused using the feedback signal from a point (guide star) at the location of interest. In Chapter 2, we studied how a focus can be formed through scattering media without access to such a localized form of feedback. The 'blind' optimization of a mixed nonlinear signal from multiple guide stars can lead to a single sharp focus. We developed and experimentally validated a model that accurately describes the statistics of this optimization process. We derived the minimum requirements that need to be satisfied to allow a focus to be formed with WFS. Once formed, the optimized focus has to be scanned over the sample to form an image. In Chapter 3, we describe the generalized optical memory effect with a theoretical framework that unifies all first-order spatial correlations of scattered light. This field-field correlation function describes how much light can be shifted and tilted inside a scattering medium while remaining correlated to its original form. Moreover, we derived and experimentally validated a model that predicts the extent of the generalized memory effect in forward scattering media, such as biological tissue. Using this model, we found the optimal focus scanning mechanism for deep-tissue microscopy.

Chapter 4 describes how we implemented feedback-based wavefront shaping to enhance the penetration depth of a two-photon excitation microscope. In a proof-of-principle experiment, we demonstrated that the signal from a fluorescent guide star hidden behind a scattering surface could be enhanced by a factor of 10 . Furthermore, in this chapter we introduced a 
new imaging scheme we call digital conjugate adaptive optics (DCAO), which makes optimal use of the generalized optical memory effect. This technique allows us to digitally project the wavefront correction to any layer inside the sample. Compared to conventional imaging schemes, DCAO allowed us to form an image over an area up to 64 times as large without having to reoptimize the focus.

In Chapter 5, we introduce model-based wavefront shaping. This new method is conceptually completely different from existing WFS methods, as model-based wavefront shaping does not rely on guide stars at all. Here, we numerically compute the optimal wavefront by modeling the light propagation through a digital refractive index model of the sample. In a proof-of-principle imaging experiment, we demonstrate the feasibility and robustness of our technique, outperforming state-of-the-art WFS methods by at least a factor of two in depth.

We developed a new numerical method for high accuracy light propagation simulations, which is described in Chapter 6. In this method, we make use of the Born series, which we modified to guarantee convergence to the analytical solution for arbitrarily large scattering structures. We show that our numerical method is orders of magnitude faster and more accurate than the current state-of-the-art. Finally, in Chapter 7, we introduce a new type of boundary conditions for light propagation simulations. By implementing these new boundary conditions, the total memory consumption of our numerical method could be reduced by as much as $84 \%$ in a three-dimensional test simulation. Our modified Born series method allows us to accurately and efficiently study light scattering in two and three-dimensional structures that were previously too large to be simulated.

\section{Outlook}

In this thesis we exclusively studied static samples. Our wavefront shaping techniques require the scattering response of the samples to remain stable for the duration of the experiment. However, living tissue is known to be dynamically scattering [1]. In our experiments, the speed of the focus optimization algorithms is limited by the refresh rate of the liquid crystal spatial light modulator (SLM). Devices such as segmented deformable mirrors [2] or acoustic optic deflectors [3] can be orders of magnitude faster at the costs of a reduced number of degrees of freedom. Alternatively, high speed scanning mirrors can be used to construct the different input modes for the measurement phase [4, 5]. Afterwards, the image can be acquired using the optimized wavefront constructed with a high-resolution SLM. Moreover, recent results indicate that iterative wavefront shaping in dynamic scattering media can lead to light coupling to modes with significantly longer decorrelation times [6], resulting in enhanced focus stability. These findings give hopeful prospects for wavefront shaping microscopy in rapidly changing biological tissue.

Decades before the invention of wavefront shaping, astronomers were using adaptive optics (AO) to improve the imaging quality of their telescopes. In the past years, many AO techniques from astronomy have crossed over to microscopy [7, 8]. Adaptive optics is in essence based on a ray model of light and is mostly concerned with smooth aberrations introduced by imperfect optical elements or slight refractive index variations. In contrast, complex wavefront shaping provides a more universal solution to light scattering, yet treats a sample as a disordered black box, assuming no prior knowledge is available. Deep tissue microscopy lies within an regime in between AO and WFS. A regime where light experiences quasi- 
ballistic propagation, and where scattering can no longer be described in terms of smooth aberrations. The cross fertilization of ideas between adaptive optics and wavefront shaping could lead to faster focus optimization algorithms and better imaging schemes. As an example, our generalized optical memory effect yields the optimal scanning scheme and arose from the gap between conjugate adaptive optics and the field-field correlations known from mesoscopic physics.

We improved the quality of our microscope images by directly controlling the scattered light. However, light scattering does not destroy the image information, but rather scrambles it in a disordered manner. Therefore, an accurate model of light propagation could be used to compensate for scattering in post-processing. In a recent paper in the field of computational microscopy, a layered Born approximation model was used for image reconstruction inside a multiple-scattering biological samples [9]. Although this method provides state-of-the-art reconstructions, their model only takes into account the first-order scattering terms for each sample sub-layer. Our modified Born series method allows us to efficiently calculate all orders of the Born series, providing a more accurate model for light scattering. Therefore, we think our numerical method might play an important role for image reconstruction and other inverse problems in more strongly scattering samples.

\section{Conclusion}

In conclusion, we extended the theory of light propagation in forward scattering media and developed a numerical tool to accurately simulate light scattering. From these new insights, we were also able to develop techniques to focus and scan scattered light for enhanced imaging in two-photon excitation microscopy. We believe our contributions to the field of wavefront shaping microscopy will enable new non-invasive imaging applications deep inside biological tissue.

\section{Bibliography}

[1] M. M. Qureshi, J. Brake, H.-J. Jeon, H. Ruan, Y. Liu, A. M. Safi, T. J. Eom, C. Yang, and E. Chung, "In vivo study of optical speckle decorrelation time across depths in the mouse brain", Biomed. Opt. Express 8, 4855-4864 (2017).

[2] B. Blochet, L. Bourdieu, and S. Gigan, "Focusing light through dynamical samples using fast continuous wavefront optimization", Opt. Lett. 42, 4994-4997 (2017).

[3] D. Feldkhun, O. Tzang, K. H. Wagner, and R. Piestun, "Focusing and scanning through scattering media in microseconds", Optica 6, 72-75 (2019).

[4] M. Cui, "A high speed wavefront determination method based on spatial frequency modulations for focusing light through random scattering media", Opt. Express 19, 29892995 (2011).

[5] I. N. Papadopoulos, J.-S. Jouhanneau, J. F. A. Poulet, and B. Judkewitz, "Scattering compensation by focus scanning holographic aberration probing (F-SHARP)", Nat. Photonics 11, 116-123 (2017). 
[6] B. Blochet, K. Joaquina, L. Blum, L. Bourdieu, and S. Gigan, "Enhanced stability of the focus obtained by wavefront optimization in dynamical scattering media", Optica 6, 1554-1561 (2019).

[7] M. J. Booth, "Adaptive optical microscopy: the ongoing quest for a perfect image", Light Sci. Appl. 3, e165 (2014).

[8] N. Ji, “Adaptive optical fluorescence microscopy”, Nat. Methods 14, 374-380 (2017).

[9] M. Chen, D. Ren, H.-Y. Liu, S. Chowdhury, and L. Waller, "Multi-layer Born multiplescattering model for 3D phase microscopy", Optica 7, 394-403 (2020). 


\section{Nederlandse samenvatting}

In biologisch weefsel is de maximale beelddiepte van een microscoop gelimiteerd door de verstrooiing van licht. In dit proefschrift onderzoeken we hoe deze fundamentele limiet kan worden doorbroken door middel van wavefront shaping (golffrontvorming). Wavefront shaping is een krachtige techniek waarmee het licht in verstrooiende media kan worden gestuurd. We vergroten de maximale beelddiepte door lichtverstrooiing in weefsel-nabootsende monsters te bestuderen en door nieuwe golffrontvormende microscooptechnieken te ontwikkelen.

In het eerste deel van dit proefschrift worden nieuwe theoretische modellen ontwikkelt voor lichtverstrooiingsverschijnselen die relevant zijn voor microscopie. In hoofdstuk 2 bestuderen we hoe licht kan worden gefocusseerd door een verstrooiend materiaal, zonder gebruik te maken van een gelokaliseerd signaal achter dit materiaal. We onderzoeken onder welke voorwaarden de 'blinde' optimalisatie van een niet-lineair signaal, afkomstig van meerdere bronnen, tot één focus kan leiden. In hoofdstuk 3 wordt het gegeneraliseerde optische geheugeneffect geïntroduceerd, dat alle eerste-orde ruimtelijke correlaties van verstrooid licht beschrijft. Deze correlatiefunctie beschrijft hoeveel een geoptimaliseerde focus kan worden verschoven en gekanteld binnen een verstrooiend medium. Uit deze correlatiefunctie wordt afgeleid hoe licht op een optimale manier kan worden gescand voor beeldvorming in voorwaarts verstrooiende media.

In het tweede deel van dit proefschrift bespreken we hoe wavefront shaping kan worden gebruikt om de prestaties van een twee-foton excitatie microscoop (TFM) te verbeteren. Hoofdstuk 4 behandelt de basisprincipes van TFM-beeldvorming en hoe de fluorescerende structuren binnen het monster kunnen worden gebruikt als signaal voor het wavefront shaping algoritme. Verder laten we zien hoe het effectieve gezichtsveld door een verstrooiende laag kunnen worden verbeterd met behulp van het gegeneraliseerde optische geheugeneffect. In hoofdstuk 5 wordt een nieuwe categorie van golffrontvormende technieken beschreven, die we modelgebaseerde wavefront shaping noemen. In plaats van het signaal van een bron binnen de verstrooiende structuur te gebruiken, gebruikt deze techniek lichtsimulaties om de golffrontcorrectie te verkrijgen. Vervolgens kan dit golffront worden gemaakt met de spatiële lichtmodulator om een scherpe focus te vormen. In een experiment vormen wij beelden van hoge resolutie op een diepte waar met conventionele microscooptechnieken niet instaat zijn om nog een signaal te detecteren.

In het derde deel van het proefschrift wordt een nieuwe numerieke methode voor het simuleren van lichtvoortplanting in grote verstrooiende media gepresenteerd. In hoofdstuk 6 laten we zien dat onze methode, gebaseerd op een gemodificeerde Born-serie, ordes van grootte sneller en nauwkeuriger is dan de huidige methoden. In hoofdstuk 7 introduceren we nieuwe randvoorwaarden voor onze numerieke methode, waarmee de nauwkeurigheid en de geheugenefficiëntie van de methode verder worden verbeterd. Met onze gemodificeerde Born-serie-methode kunnen we lichtverstrooiing nauwkeurig bestuderen in twee- en driedi- 
mensionale structuren die voorheen te groot waren om te simuleren.

Samenvattend hebben we de theorie van lichtvoortplanting in voorwaartse verstrooiingsmedia uitgebreid en een nieuwe numerieke methode geïntroduceerd om lichtverstrooiing nauwkeurig te kunnen simuleren. Vanuit deze nieuwe inzichten hebben we technieken ontwikkeld om verstrooid licht te kunnen focusseren en scannen voor de verbeterde beeldvorming in een twee-foton excitatie microscoop. Wij verwachten dat onze bijdragen op het gebied van wavefront shaping-microscopie nieuwe niet-invasieve beeldvormingstoepassingen diep in het biologische weefsel mogelijk zullen maken. 


\section{Acknowledgements}

This is the final section of the thesis. My compliments to you if you made it this far through the book, and welcome to those that directly skipped to this section. Many kind and supportive individuals have helped me during my $\mathrm{PhD}$ project. Here I would like to express my gratitude to them.

First, I want to thank Wiendelt Steenbergen for giving me the opportunity to work at the Biomedical Photonic Imaging group. Thank you for your support and advise during our meetings, and thank you for taking care of the (somewhat cumbersome) TGS administrative tasks.

The support staff was crucial for my experimental work. Johan, I appreciate your help with electronics and optics, and thank you for making those fancy Solid Works drawings. Wilma, working together with you in the chemical lab has always been a lot of fun. If I ever have to deal with another human placenta in a bucket, then I now know who to turn to. Tommie! Thank you for all your hard work and your endless optimism. Aligning the microscope together with you to the sounds of Muse were probably the best days in the lab. As cofounder of the two-photon microscope, you now have the responsibility to continue its legacy.

Next up are my office roomies: Pim, Onno, Sjoukje, Tzu-lun and Hanna. Thank you all for contributing to the office that clearly has the nicest atmosphere (especially during Christmas). Sjoukje, thank you for the motivational cat pictures and teaching me to care about layout and design. Onno, thanks for distracting me with puzzles and the 'coffee break' board games. I am sorry for misspelling your last name all the time. Tzu-lun, I appreciate your infectious enthusiasm and all the nice Asian snacks that you brought to the office.

After spending 5 years at BMPI, I can say with certainty that you guys are the coolest group in the world! Yes! Yes! Yes! Over the past years we have done so many great activities together like karaoke nights, building a telescope, visiting an opera, beer brewing. It is impossible to list all of the fun things that we did together. To David, Maura, Sjoukje, Yoeri en Colin my special thanks for an awesome road trip in California. I am also very grateful to 'Ivo's people' (Abhilash, Bahareh, Daniël, Giulia, Lyuba, Tom and Tzu-lun) for being such a pleasant and helpful team. It has been great being part of such a warm and welcoming research group. Thank you all for all the great memories. Pew! Pew!

Ik wil ook graag mijn familie bedanken voor hun steun. In het bijzonder Liz, die elk van mijn kleine probleempjes en dilemma's heeft moeten aanhoren. Lizzy, dank je voor de liefde die je me elke dag weer geeft.

Special thanks to Saroch, Roarke, Ioannis, Benjamin, Lyuba, Abhilash, Tom and Maaike for their direct contributions to the chapters in this thesis. Extra kudos to Abhilash, Daniël, David, Giulia, Liz and Tom for proofreading parts of my thesis during the final months of the writing process. I would also like to express my gratitude to my thesis committee members 
for all the energy and time spent on reading this thesis and preparing for the defense.

Finally, this work would not have been possible without the help from my supervisor Ivo Vellekoop. Ivo, I loved our lengthy and nerdy discussions about 8-dimensional correlation functions, nonlinear random matrix theory and the like. Thank you so much for taking the time to help me with coding, writing, doing experiments, and so many other things. Working together with you has really boosted my self-confidence and has taught me how much fun science can be. 